\title{
Pathway crosstalk analysis in prostate cancer based on protein-protein network data
}

\author{
H. Y. LI, N. JIN, Y. P. HAN, X. F. JIN* \\ Department of Urology, China-Japan Union Hospital of JiLin University, 126 Xiantai ST, Chang Chun, JiLin. 130033, China \\ ${ }^{*}$ Correspondence: jinxuefeiei@hotmail.com
}

Received March 9, 2016 / Accepted July 18, 2016

\begin{abstract}
Prostate cancer (PCa) is one of the major leading cause in men and no effective biomarkers or therapy have been approved for it to date. This study aimed to explore the molecular mechanisms and identify the potential molecular biomarkers of PCa.

The microarray profile GSE38241 including 18 prostate cancer metastasis and 21 normal prostate samples was retrieved from the Gene Expression Omnibus (GEO) database. Differentially expressed genes (DEGs) were identified by Limma. DEGs functions were investigated by Gene Ontology $(\mathrm{GO})$ and pathway enrichment analysis. Moreover, protein-protein interaction (PPI) network of DEGs was constructed, followed by functional analysis of modules. Additionally, pathway crosstalk network was constructed by integrating PPI network and Kyoto encyclopedia of genes and genomes (KEGG) pathways.

Totally, 334 up - and 703 down-regulated DEGs were identified. The functions of up-regulated DEGs were significantly enriched in GO terms of cell cycle phase and cell cycle process. While down-regulated DEGs mainly participated in actin filament-based process. Among these pathways in the pathway crosstalk network, T cell receptor signaling pathway, chemokine signaling pathways, endometrial cancer and glioma were found to play critical roles during PC progression.

Cell division cycle 45 (CDC45), baculoviral IAP repeat containing 5 (BIRC5) and cell division cycle associated 5 (CDCA5) may be useful markers for predicting tumor metastasis and therapeutic targets for the treatment of PCa patients. Moreover, the pathway crosstalk network provides the groundwork that targeting multiple pathways might be more effective than targeting one pathway alone.
\end{abstract}

Key words: prostate cancer, differentially expressed genes, protein-protein interaction network, pathway crosstalk

Prostate cancer $(\mathrm{PCa})$ is a kind of malignancy resulting from the pathological changes in men's prostate tissue [1]. PCa is the most commonly diagnosed cancer for men in American and is by far the second leading cause of cancer-related death in men [2]. Moreover, recurrence rate remains about $15 \%$ within 5 years after prostatectomy and approximately $40 \%$ within 10 years [3]. The mortality rate of PCa patients is higher even after prostatectomy, hormone therapy or radiation [4]. Despite the high incidence rate as well as high mortality rate, the etiology of PCa still remains largely unknown. Thus, more efforts should be devoted for exploring the molecular mechanism and identifying molecular targets to prevent $\mathrm{PCa}$ metastases during the early stage to improve the prognosis of PCa patients.

Recently, considerable researches were undertaken to explore the molecular mechanism of PCa. Some genes have been identified to be aberrant in PCa. The six-transmembrane epi- thelial antigen of prostate 1 (STEAP1) gene overexpression has been reported in PCa [5]. Recently, Neto et al. have reported a significant, direct link between human epidermal growth factor receptor 2 (HER2)/neu over-expression and recurrence and death risk in PCa patients [4]. Additionally, the mutation in $\beta$-catenin has been reported in $\mathrm{PCa}$ [6]. A previous study has suggested that insulin-like growth factor-1 (IGF-1) signaling involved in PCa development through blocking apoptosis and promoting cancer cell proliferation and invasion $[7,8]$. Recent study has demonstrated that focal adhesion pathway is of special importance to the development and the progression of human PCa [9]. Additionally, Wnt/ $\beta$-catenin is one of the most influential pathways on both human and rat $\mathrm{PCa}$ cell growth [10]. However, PCa complexity is not only the combination of proteins in the pathways but also the interactions of the crosstalk of these pathways $[11,12]$. Recently, the cross-talk among different signaling pathways has been 
demonstrated to play a crucial role in PCa: the inhibition of IGF- 1 reduced the $\beta$-catenin signaling. Wnt $/ \beta$-catenin inhibition subsequently resulted in an inhibition of the T-cell factor/ lymphoid enhancer factor dependent transcriptional activity and effective inhibition of PCa cell growth [13]. The cooperation of Wnt signaling pathway and Notch signaling pathway is also found in $\mathrm{PCa}$ [14].

In the current study, microarray data GSE38241 [15] was employed to identify differentially expressed genes (DEGs) between PCa metastasis and normal prostate samples. Through some studies have analyzed the datasets for biomarkers and small molecules drugs of metastatic prostate carcinoma ${ }^{[9,16]}$, a large amount of information in the dataset still remain to be discovered. In this study, in addition to the Gene Ontology (GO) and pathway enrichment analysis of DEGs, the protein-protein interaction (PPI) network was constructed, followed by functional analysis of modules. Additionally, the pathway crosstalk network was constructed among PCa-related pathways by integrating PPI and Kyoto encyclopedia of genes and genomes (KEGG) pathways information. Therefore, this research will light the further understanding of molecular mechanisms of PCa. Meanwhile, this may provide new insight into the therapy for it.

\section{Materials and methods}

Data source. The mRNAs expression profile of GSE38241 [15] which was generated by Aryee et al. based on GPL4133 (Agilent-014850 Whole Human Genome Microarray 4x44K G4112F (Feature Number version) was downloaded from the Gene Expression Omnibus (GEO) database (http://www.ncbi. nlm.nih.gov/geo/). A total of 39 mRNA chips were available, including 18 PC metastasis samples and 21 normal prostate samples. The raw data files and the probe annotation files were obtained and used for further analysis.

Data preprocessing. The probe ID was converted into gene symbol based on the annotation of the platform. Probes with at least one defective expression values were abandoned. For each sample, the expression values of all probe set that map to a given gene were reduced and averaged to a single value. Then, the data were normalized using PreprocessCore package [17]. Finally, the gene expression matrix was obtained.

Identification of DEGs. Based on data preprocessing, the limma package [18] in R language was performed to identify DEGs between PCa metastasis samples and normal prostate samples. $\mid \log _{2}$ fold change (FC) $\mid>1$ and FDR $<0.05$ were used as the cut-off criterion of DEGs. The raw $p$ values were adjusted to false discovery rates (FDR) on the basis of Benjamini \& Hochberg method [19].

GO and pathway enrichment analysis of DEGs. According to GO Database (http://www.geneontology.org/), GO annotation was performed to analyze the functions of DEGs between PCa metastasis samples and normal prostate samples. GO analysis has been used as functional enrichment studies of large-scale genes frequently [20]. GenCLiP 2.0, which is a web-based text-mining server for gene clustering and molecular networks construction, was applied to perform the GO analysis [21]. A p $\leq 0.05$ and Hit $\geq 10$ were the cutoff criterions for $\mathrm{GO}$ categories.

KEGG databases cover all kinds of biochemistry pathways [22]. GenCLiP 2.0 was applied for KEGG pathway enrichment analysis of DEGs. A p $\leq 0.05$ was chosen as the cut off value.

PPI network construction and functional analysis of modules. The Human Protein Reference Database (HPRD) [23] is a protein database accessible through the internet. A total of 39240 PPI pairs were obtained from HPRD. The Search Tool for the Retrieval of Interacting Genes (STRING) [24] database offers both experimental and predicted interaction information. STRING database was applied to construct the PPI network.

Then, the edges and nodes of the PPI network were so complicated, there is a need for further analysis to expose the enriched functional modules of the PPI network using Cytoscape and ClusterOne [25, 26]. Finally, GO and pathway enrichment analysis of modules was performed.

Significance analysis of pathway crosstalk under PPI and KEGG data. All human pathways were collected from KEGG database. The pathway of PCa was screened as host pathway. The candidate pathways were obtained with at least a protein overlap between any given pair of pathways.

Pearson correlation coefficient (PCC) was applied to calculate the similarity of gene expression and weight pairwise gene co-expression in PCa. Then, $\mathrm{p}$-values mapping to nodes and edges in the PPI network were calculated and obtained. The following formula [27] was performed to assess and measure statistical significance of functional interactions by a scoring scheme.

$$
\begin{aligned}
& S(e)=f(\operatorname{diff}(x), \operatorname{corr}(x, y), \operatorname{diff}(y)) \\
& =-2 \sum_{i=1}^{k} \log _{e}\left(p_{i}\right)
\end{aligned}
$$

Where the $\operatorname{diff}(\mathrm{x})$ and $\operatorname{diff}(\mathrm{y})$ represented the quantitative assessment in differential gene expression of gene $\mathrm{x}$ and gene $y$, respectively; corr $(\mathrm{x}, \mathrm{y})$ represented their correlation strength between gene $\mathrm{x}$ and gene $\mathrm{y}$ based on the expression levels; f represented a general method in data integration taking account of multiple data sets across a variety of statistical power; Where $\mathrm{k}=3, \mathrm{p} 1$ and $\mathrm{p} 2$ indicated the statistical significance ( $p$-values) of differential expression of two nodes, while $\mathrm{p} 3$ referred to their co-expression strength ( $p$-value).

To determine the significance of a pathway $\mathrm{P}$, the scores of all edges $S(e)$ of every pathway were summarized as $\mathrm{Sp}$. To assess statistical significance of this pathway, $p$-values were obtained and calculated from 1000 randomly generated pathways with the same size. Score frequency that larger than $\mathrm{Sp}$ was used as the significant $\mathrm{p}$-value of pathway $\mathrm{P}$ to describe its importance. We considered the pathway with the p-value $<0.05$ as the significant pathway. 
Disease Ontology (DO) annotation of DEGs. To identify a direct link between human cancer and DEGs, TargetMine (http://targetmine.nibio.go.jp/) which is an integrated data warehouse for known disease-associated genes was used for retrieval of candidate target genes and proteins for cancer from large-scale experiments. Additionally, DEGs have been reported to play roles in cancer pathogenesis would be identified to validate the results.

\section{Results}

Identification of DEGs. Heatmap in Figure 1 displayed the differential gene expression between prostate cancer metastasis and normal prostate samples (Supplemental Table 1). After preprocessing, 1037 DEGs were identified, including 334 up - and 703 down-regulated DEGs (Supplemental Table 2). Alpha-methylacyl-CoA racemase (AMACR, $\log _{2} \mathrm{FC}=3.263$ ) and actin, gamma 2 , smooth muscle, enteric (ACTG2, $\log _{2} \mathrm{FC}=-5.945$ ) had the highest fold changes among the up - and down-regulated genes, respectively.

GO and pathways enriched by DEGs. The top 3 clusters of GO terms which were most significantly enriched by DEGs were shown in Table 1. Up-regulated DEGs were significantly enriched in cell cycle phase $(\mathrm{p}=4.56 \mathrm{E}-12)$, chromosome segregation $(\mathrm{p}=1.80 \mathrm{E}-10)$ and cell cycle process $(\mathrm{p}=2.49 \mathrm{E}-08)$.
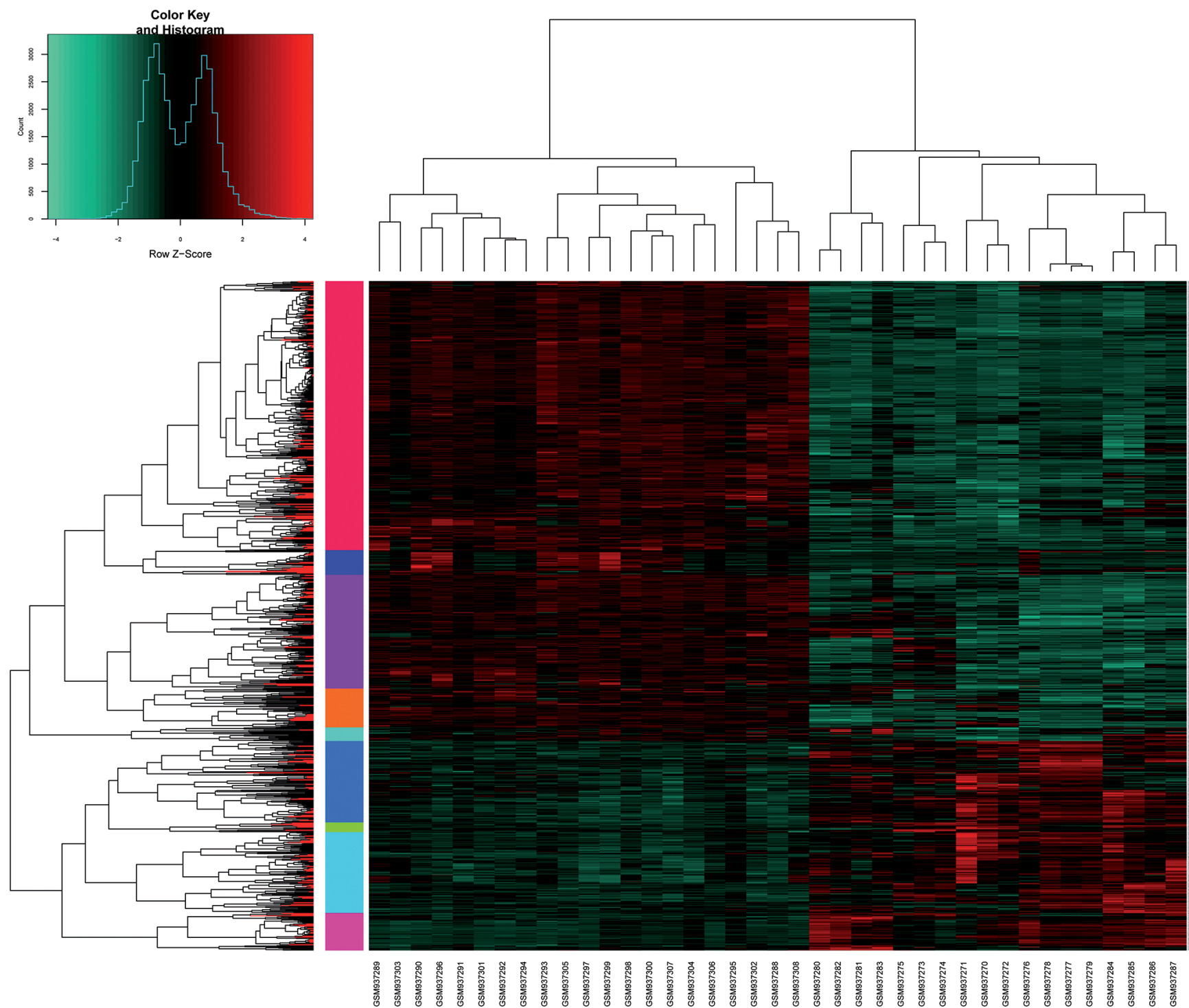

Figure 1. Heatmap of differential gene expression between prostate cancer metastasis and normal prostate samples. Each row represents the relative levels of expression of a single gene across all samples; each column represents the levels of expression for a single sample. The colors red and green denote high and low expression, respectively. 
While down-regulated DEGs mainly participated in muscle contraction $(\mathrm{p}=4.39 \mathrm{E}-81)$, muscle system process $(\mathrm{p}=$ 2.61E-76), muscle structure development $(\mathrm{p}=6.46 \mathrm{E}-48)$ and cardiovascular system development $(\mathrm{p}=3.17 \mathrm{E}-31)$.

The KEGG pathways obtained with $\mathrm{p} \leq 0.05$ of the up and down-regulated genes were shown in Table 1. On the basis of the results, the up-regulated DEGs were enriched in pathways significantly such as leishmania infection $(\mathrm{p}=$ $3.45 \mathrm{E}-07)$, antigen procession and presentation $(\mathrm{p}=1.09 \mathrm{E}-$ $05)$ and type I diabetes mellitus $(\mathrm{p}=5.11 \mathrm{E}-05)$. Besides, the down-regulated DEGs were significantly enriched in dilated cardiomyopathy $(\mathrm{p}=5.24 \mathrm{E}-14)$, hypertrophic cardiomyopathy $(\mathrm{HCM})(\mathrm{p}=8.67 \mathrm{E}-14)$ and arrhythmogenic right ventricular cardiomyopathy (ARVC) $(\mathrm{p}=1.36 \mathrm{E}-07)$. These pathways of the down-regulated DEGs were mainly associated with cardiomyopathy. The detailed information of DEGs enriched in GO terms and pathways were shown in Supplemental Table 3.

PPI and functional analysis of modules. To identify the connection between DEGs and distinct functional modules, the STRING tool was performed to obtain the PPI network of DEGs. The PPI network as well as module of up-regulated DEGs was shown in Figure 2. DEGs highly connected (known as high degree nodes) to others would be more important in the network. While gene modules function as conserved molecular "circuits" responsible for specific biological function. In the module, there were 41 nodes and 713 edges. The density of the module was 0.575 . Additionally, the PPI network and module of down-regulated DEGs was shown in Figure 3. In the module, there were 39 nodes and 311 edges. The density of the module was 0.308 .

We then performed the GO and KEGG pathway enrichment analysis of DEGs in modules. Similarly, the top three

Table 1. The top 3 cluster Gene Ontology (GO) functional enrichment and Kyoto Encyclopedia of Genes and Genomes (KEGG) pathway of differentially expressed genes.

\begin{tabular}{|c|c|c|c|c|}
\hline Category & & GO term & Hit & p value \\
\hline \multirow{7}{*}{$\begin{array}{l}\text { GO terms of } \\
\text { up-regulated } \\
\text { genes }\end{array}$} & \multirow{2}{*}{ Cluster 1 Enrichment Score: 10.40} & Cell cycle phase & 24 & $4.56 \mathrm{E}-12$ \\
\hline & & Mitotic cell cycle & 29 & $3.45 \mathrm{E}-10$ \\
\hline & \multirow{3}{*}{ Cluster 2 Enrichment Score: 7.98} & Chromosome segregation & 12 & $1.80 \mathrm{E}-10$ \\
\hline & & Condensed chromosome & 11 & $3.14 \mathrm{E}-07$ \\
\hline & & Chromosome,centromeric region & 11 & $2.05 \mathrm{E}-08$ \\
\hline & \multirow{2}{*}{ Cluster 3 Enrichment Score: 7.20} & Cell cycle process & 38 & $2.49 \mathrm{E}-08$ \\
\hline & & Cell cycle & 11 & $1.60 \mathrm{E}-07$ \\
\hline \multirow{10}{*}{\multicolumn{2}{|c|}{ Pathways of up-regulated genes }} & Leishmania infection & 7 & $3.49 \mathrm{E}-07$ \\
\hline & & Allograft rejection & 5 & $2.61 \mathrm{E}-04$ \\
\hline & & Antigen & 7 & $1.09 \mathrm{E}-05$ \\
\hline & & Type I diabetes mellitus & 6 & $5.11 \mathrm{E}-05$ \\
\hline & & Asthma & 5 & $9.30 \mathrm{E}-05$ \\
\hline & & Viral myocarditis & 5 & $1.07 \mathrm{E}-03$ \\
\hline & & Graft versus host disease & 5 & $4.26 \mathrm{E}-04$ \\
\hline & & Intestinal immune network for IgA production & 5 & $8.92 \mathrm{E}-04$ \\
\hline & & Autommune thyroid disease & 5 & $1.29 \mathrm{E}-03$ \\
\hline & & Systemic lupus erythematosus & 6 & $2.20 \mathrm{E}-02$ \\
\hline \multirow{9}{*}{$\begin{array}{l}\text { GO terms of } \\
\text { down-regulated } \\
\text { genes }\end{array}$} & \multirow{3}{*}{ Cluster 1 Enrichment Score: 56.04} & Muscle contraction & 70 & $4.39 \mathrm{E}-81$ \\
\hline & & Muscle system process & 73 & $2.61 \mathrm{E}-76$ \\
\hline & & System process & 143 & $6.73 \mathrm{E}-13$ \\
\hline & \multirow{3}{*}{ Cluster 2 Enrichment Score: 31.11} & Muscle structure development & 79 & $6.46 \mathrm{E}-48$ \\
\hline & & Tissue development & 129 & $3.26 \mathrm{E}-25$ \\
\hline & & Organ development & 181 & $2.17 \mathrm{E}-22$ \\
\hline & \multirow{3}{*}{ Cluster 3 Enrichment Score: 25.25} & Circulatory system development & 92 & $3.17 \mathrm{E}-31$ \\
\hline & & Cardiovascular system development & 92 & $3.17 \mathrm{E}-31$ \\
\hline & & Anatomical structure formation involved in morphogenesis & 78 & $1.76 \mathrm{E}-15$ \\
\hline \multirow{4}{*}{\multicolumn{2}{|c|}{ Pathways of down-regulated genes }} & Dilated cardiomyopathy & 19 & $5.24 \mathrm{E}-14$ \\
\hline & & Hypertrophic cardiomyopathy (HCM) & 18 & $8.67 \mathrm{E}-14$ \\
\hline & & Arrhythmogenic right ventricular cardiomyopathy (ARVC) & 13 & $1.36 \mathrm{E}-07$ \\
\hline & & Cardiac muscle contraction & 9 & $4.87 \mathrm{E}-03$ \\
\hline
\end{tabular}


clusters of GO terms and pathways enriched by up - and down-regulated DEGs were shown in Table 2. The detailed information were shown in Supplemental Table 4.

The up-regulated DEGs, such as cell division cycle 45 (CDC45), baculoviral IAP repeat containing 5 (BIRC5) and cell division cycle associated 5 (CDCA5), were significantly enriched in GO functions of cell cycle phase ( $\mathrm{p}=1.10 \mathrm{E}-88)$, mitotic cell cycle $(\mathrm{p}=7.82 \mathrm{E}-88)$ and cell cycle process $(\mathrm{p}=$ 6.84E-77). The up-regulated DEGs were significantly enriched in pathways such as cell cycle $(\mathrm{p}=6.09 \mathrm{E}-05)$ and DNA replication $(\mathrm{p}=6.61 \mathrm{E}-06)$. While the down-regulated DEGs mainly participated in GO terms of actin filament-based process $(\mathrm{p}=$ 4.53E-130), cytoskeletal protein binding $(\mathrm{p}=5.69 \mathrm{E}-92)$ and myofibril ( $\mathrm{p}=4.02 \mathrm{E}-54)$, and remarkably enriched in pathways such as dilated cardiomyopathy $(\mathrm{p}=7.19 \mathrm{E}-13)$, hypertrophic cardiomyopathy $(\mathrm{HCM})(\mathrm{p}=1.56 \mathrm{E}-11)$ and cardiac muscle contraction $(\mathrm{p}=4.68 \mathrm{E}-07)$.

Significance analysis of pathway crosstalk under PPI and KEGG data. To identify the important pathways altered in PCa, a statistical method on pathway level was used. The significance analysis of crosstalk effects in pathways was based on the PPI database and KEGG database. The molecular crosstalk between host and candidate pathways showed that many significant pathways containing $\mathrm{T}$ cell receptor signaling pathway, chemokine signaling pathway, endometrial cancer and glioma were obtained (Figure 4). This results indicated the connection between immune and PC, and also suggested the commonness of cancer. Addi-

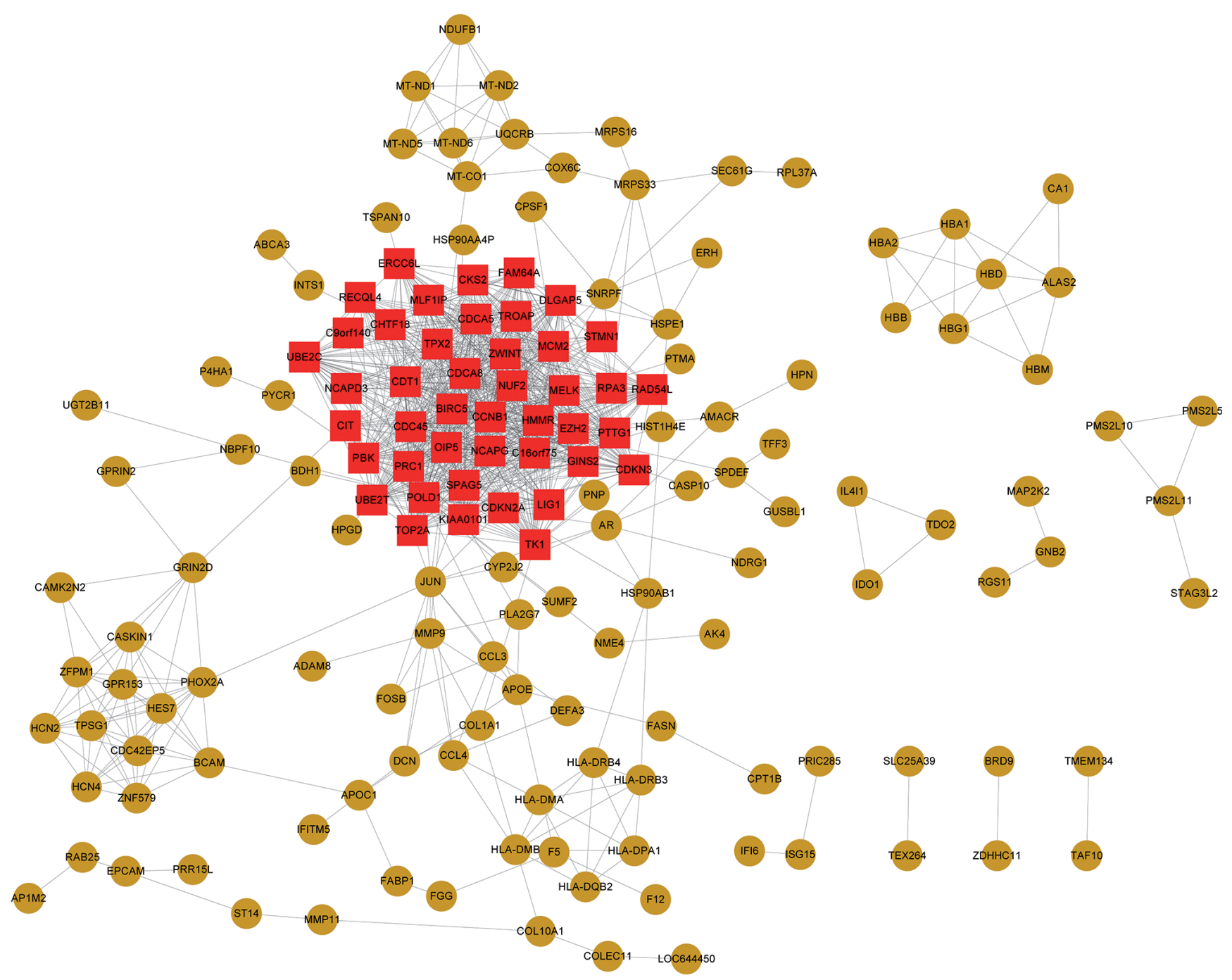

Figure 2. Protein-protein interaction (PPI) network of up-regulated differentially expressed genes. The red square nodes represent the module of the PPI network. Other nodes represent up-regulated differentially expressed genes outside the module. In the module, there were 41 nodes and 713 edges. The density of the module was 0.575 . 
tionally, the associations between DEGs and pathways were provided in Supplemental Table 5.

DO terms of DEGs. Multiple DO terms of DEGs were obtained (as shown in Supplemental Table 6). Especially, the cancer-related terms including PCa, carcinoma, cell type cancer and malignant glioma were enriched by down-regulated DEGs, besides, cancer, malignant ovarian surface epithelialstromal neoplasm, ovary epithelial cancer, and ovarian carcinoma were enriched by up-regulated DEGs.

\section{Discussion}

PCa is a highly prevalent cancer in older men of the western countries [28]. In the current study, we employed bioinformatics methods to explore the molecular mechanisms and identify the potential molecular biomarkers to PCa. Our results suggested that 334 up - and 703 downregulated DEGs were screened out. Moreover, the functions of up-regulated DEGs were significantly enriched in cell cycle phase and cell cycle process. While down-regulated DEGs mainly participated in actin filament-based process and dilated cardiomyopathy.

In our study, we found DEGs were mostly enriched in GO terms of cell cycle phase, cell cycle process and cell cycle. It is well known that cancer is characterized by uncontrolled cell proliferation due to dysregulation of the cell-cycle [29]. Moreover, replication of damaged DNA leads to mutations in the genome and the development of diseases such as cancer

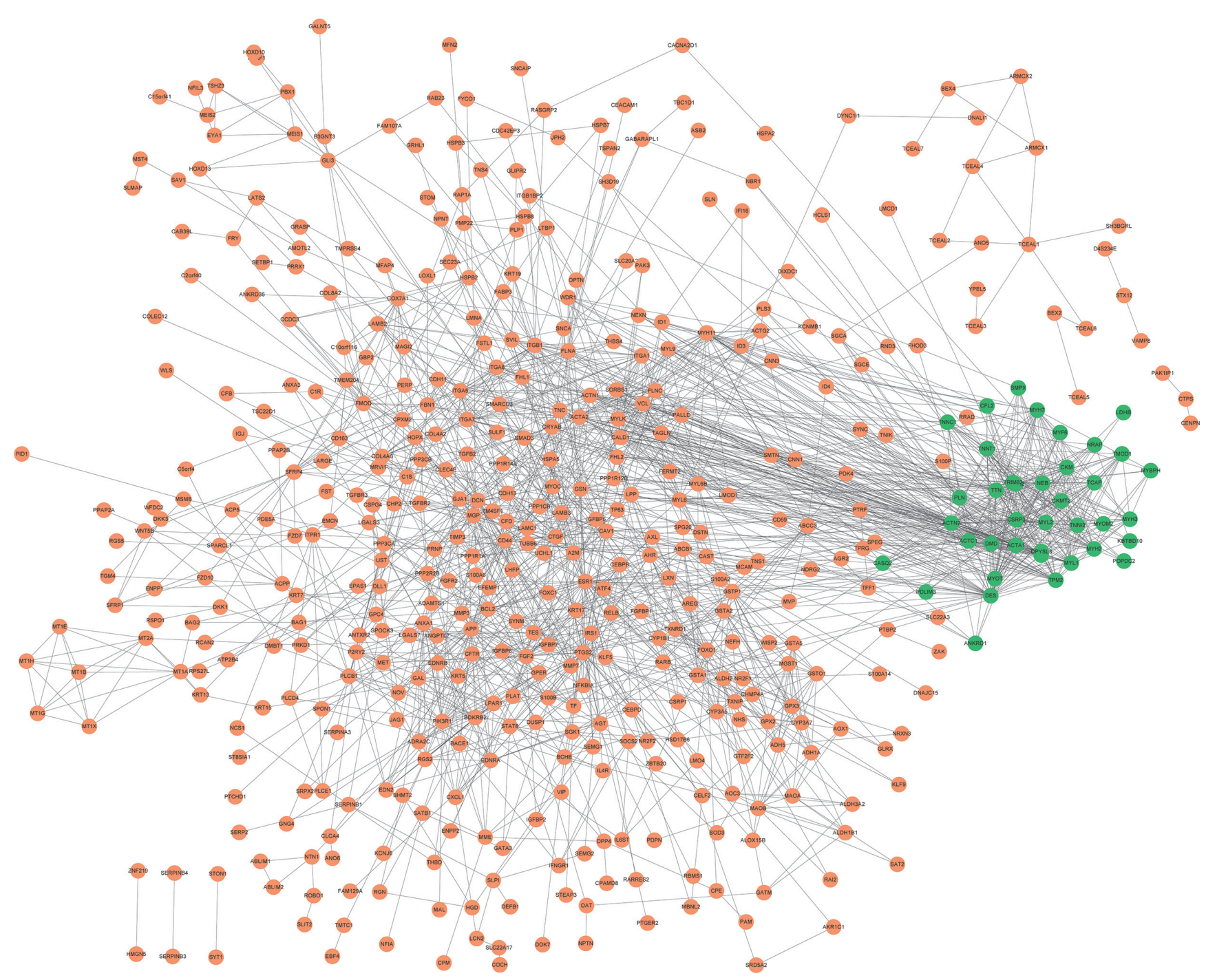

Figure 3. The protein-protein interaction (PPI) network of down-regulated differentially expressed genes.

The green circle nodes represent the module of the PPI network. Other nodes represent down-regulated differentially expressed genes outside the module. In the module, there were 39 nodes and 311 edges. The density of the module was 0.308 . 
[30]. CDC45, BIRC5, CDCA5 as DEGs were shown to be significantly up-regulated in PCa metastases samples in the present study. CDC45 plays an important role in the initiation as well as the extension of DNA replication [31, 32], and participated in the pathway of DNA damage-dependent signal transduction [33]. A former study has demonstrated that CDC45 expression level is strongly higher in human cancerderived cells than in primary human cells [34]. BIRC5 gene has been reported to be associated with PCa previously [35, 36]. Our result was in accordance with previous study. Additionally, DO term annotation revealed that multiple DEGs played roles in various cancers including PCa. This may confirm the result accuracy to some extent as well. In addition, the CDCA5 gene was suggested to play some roles in cell cycle progression via the interaction with chromatin in some immortalized cell lines [37]. Besides, Nguyen et al. have indicated that CDCA5 is an oncogene that is highly expressed in lung cancers [38]. These results strongly suggested that dysregulation of these genes related with cell cycle might be crucial for the progression of $\mathrm{PCa}$.

In the pathway crosstalk network, several significant immunity and cancer-related pathways crosstalk with $\mathrm{PCa}$, such as $\mathrm{T}$ cell receptor signaling pathway and chemokine signaling pathway. The $\mathrm{T}$ cell receptor plays a key role in the immune system. Immunotherapy of cancer is dependent on a proper target antigen and antigen presentation to the patient's immune system. Antigen presenting cells are responsible for uptake, processing and presentation of anti-

Table 2. The Gene Ontology (GO) functional enrichment and Kyoto Encyclopedia of Genes and Genomes (KEGG) pathway analysis of differentially expressed genes.

\begin{tabular}{|c|c|c|c|c|}
\hline \multicolumn{2}{|l|}{ Category } & \multirow{2}{*}{$\begin{array}{l}\text { Term } \\
\text { Cell cycle phase }\end{array}$} & \multirow{2}{*}{$\frac{\text { Hit }}{23}$} & \multirow{2}{*}{$\frac{\text { P values }}{1.10 \mathrm{E}-88}$} \\
\hline \multirow{11}{*}{$\begin{array}{l}\text { GO terms of } \\
\text { up-regulated } \\
\text { genes }\end{array}$} & \multirow{4}{*}{ Cluster 1 Enrichment Score: 79.07} & & & \\
\hline & & Mitotic cell cycle & 28 & $7.82 \mathrm{E}-88$ \\
\hline & & Cell cycle process & 34 & $6.84 \mathrm{E}-77$ \\
\hline & & Cell cycle & 36 & $9.22 \mathrm{E}-66$ \\
\hline & \multirow{4}{*}{ Cluster 2 Enrichment Score: 53.78} & Cell division & 20 & $3.49 \mathrm{E}-61$ \\
\hline & & Mitosis & 18 & $2.26 \mathrm{E}-53$ \\
\hline & & Nuclear division & 18 & $2.26 \mathrm{E}-53$ \\
\hline & & Organelle fission & 18 & $4.20 \mathrm{E}-50$ \\
\hline & \multirow{3}{*}{ Cluster 3 Enrichment Score: 36.23} & Chromosome organization & 20 & $4.52 \mathrm{E}-38$ \\
\hline & & Chromosome & 18 & $1.48 \mathrm{E}-35$ \\
\hline & & Chromosomal part & 17 & $3.05 \mathrm{E}-37$ \\
\hline \multirow{5}{*}{\multicolumn{2}{|c|}{ Pathways of up-regulated genes }} & Cell cycle & 5 & $6.09 \mathrm{E}-05$ \\
\hline & & DNA replication & 4 & $6.61 \mathrm{E}-06$ \\
\hline & & Mismatch repair & 3 & $6.60 \mathrm{E}-05$ \\
\hline & & Nucleotide excision repair & 3 & $4.70 \mathrm{E}-04$ \\
\hline & & Homologousre combination & 3 & $1.21 \mathrm{E}-04$ \\
\hline \multirow{8}{*}{$\begin{array}{l}\text { GO terms of } \\
\text { down-regulated } \\
\text { genes }\end{array}$} & \multirow{2}{*}{ Cluster 1 Enrichment Score: 80.41} & Actin filament-based process & 27 & $4.53 \mathrm{E}-130$ \\
\hline & & Cellular component movement & 22 & $3.32 \mathrm{E}-32$ \\
\hline & \multirow{4}{*}{ Cluster 2 Enrichment Score: 53.87} & Cytoskeletal protein binding & 26 & $5.69 \mathrm{E}-92$ \\
\hline & & Myofibril & 30 & $4.02 \mathrm{E}-54$ \\
\hline & & Contractile fiber & 30 & $3.09 \mathrm{E}-53$ \\
\hline & & Actin binding & 17 & $4.53 \mathrm{E}-19$ \\
\hline & \multirow{2}{*}{ Cluster 3 Enrichment Score: 46.94} & Sarcomere & 27 & $2.80 \mathrm{E}-48$ \\
\hline & & Contractile fiber partChromosomal part & 27 & $4.72 \mathrm{E}-47$ \\
\hline \multirow{8}{*}{\multicolumn{2}{|c|}{ Pathways of down-regulated genes }} & Dilated cardiomyopathy & 10 & $7.19 \mathrm{E}-13$ \\
\hline & & Hypertrophic cardiomyopathy (HCM) & 9 & $1.56 \mathrm{E}-11$ \\
\hline & & Cardiac muscle contraction & 6 & $4.68 \mathrm{E}-07$ \\
\hline & & Tight junction & 5 & $1.49 \mathrm{E}-04$ \\
\hline & & Viral myocarditis & 4 & $1.53 \mathrm{E}-04$ \\
\hline & & Arrhythmogenic right ventricular cardiomyopathy (ARVC) & 3 & $3.03 \mathrm{E}-03$ \\
\hline & & Arginine and proline metabolism & 2 & $1.93 \mathrm{E}-02$ \\
\hline & & Calcium signal pathway & 3 & $3.24 \mathrm{E}-02$ \\
\hline
\end{tabular}




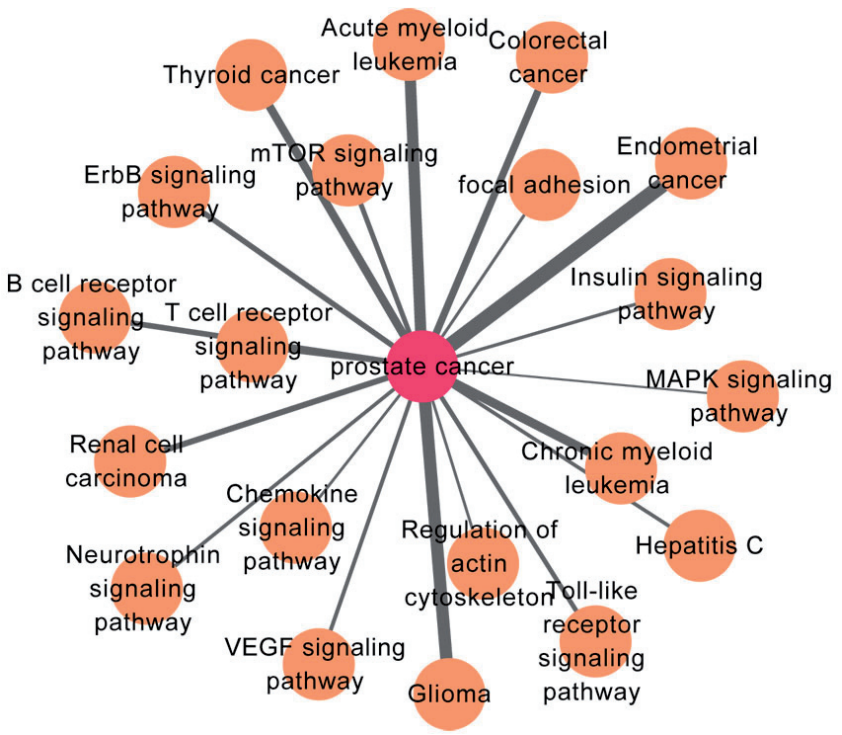

Figure 4. The pathway-crosstalk network of prostate cancer.

The rose red node in the middle represents the host pathway of prostate cancer; Peripheral orange nodes are the pathways interacting with the host pathway; The thickness of the edges is proportional to the significance between two interaction pathways.

gens to $\mathrm{T}$ cells [39]. Chemokines are shown to play roles in regulating recruitment of immune cells during inflammatory responses [40]. Considerable evidence has also showed that the chemokine signaling pathway plays crucial roles in the tumor progression and microenvironment [41]. Moreover, chemokines as well as their receptors exhibit differential expression following PCa progression [42]. Thus, a combination of targeting multiple pathways might be a promising therapy strategy for PCa.

Additionally, we found several cancer-related pathways crosstalk with PCa, such as endometrial cancer and glioma. Recent studies found the alteration of tumor suppressor gene named PTEN or MMAC1 in human PCa cell lines obtained from metastatic tissues [43, 44]. Mutations of PTEN/MMAC1 have also been reported in endometrial carcinoma [45] and glioma [46]. Moreover, the mutations in this gene are frequent in endometorial carcinoma but rare in other common gynecological cancers [47]. Suzuki et al. have demonstrated that alteration of PTEN/MMAC1 might play an important role in the pathogenesis of many human malignancies [48]. In light of these results, we infer that PTEN/MMAC1 may be a biomarker for metastatic $\mathrm{PCa}$, endometrial cancer and glioma.

In conclusion, the identified DEGs, especially CDC45, BIRC5 and CDCA5, may be pivotal genes for PCa, and these genes related with cell cycle may be useful markers for predicting tumor metastasis and therapeutic targets for the treatment of PCa patients. Moreover, B cell receptor signaling pathway, $\mathrm{T}$ cell receptor signaling pathway, endometrial cancer and glioma were found to play important roles during PCa progression in the crosstalk network among PCa-related pathways. Our studies shed new light on the mechanism and treatment of PCa. However, the consequence described above, not only genes, but also pathways associated with PCa will be evaluated and verified conjointly through animal and clinical experiments in later work.

Supplementary information is available in the online version of the paper.

\section{References}

[1] DEMARZO AM, NELSON WG, ISAACS WB, EPSTEIN JI. Pathological and molecular aspects of prostate cancer. Lancet 2003; 361: 955-964. http://dx.doi.org/10.1016/S0140-6736(03)12779-1

[2] DE BONO JS, LOGOTHETIS CJ, MOLINA A, FIZAZI K, NORTH S et al. Abiraterone and increased survival in metastatic prostate cancer. N Engl J Med 2011; 364: 1995-2005. http://dx.doi.org/10.1056/NEJMoa1014618

[3] HAN M, PARTIN AW, ZAHURAK M, PIANTADOSI S, EPSTEIN JI et al. Biochemical (prostate specific antigen) recurrence probability following radical prostatectomy for clinically localized prostate cancer. J Urol 2003; 169: 517-523. http://dx.doi.org/10.1016/S0022-5347(05)63946-8

[4] COOPERBERG MR, VICKERS AJ, BROERING JM, CARROLL PR. Comparative risk-adjusted mortality outcomes after primary surgery, radiotherapy, or androgen-deprivation therapy for localized prostate cancer. Cancer 2010; 116: 5226-5234. http://dx.doi.org/10.1002/cncr.25456

[5] HUBERT RS, VIVANCO I, CHEN E, RASTEGAR S, LEONG K et al. STEAP: a prostate-specific cell-surface antigen highly expressed in human prostate tumors. Proc Natl Acad Sci USA 1999; 96: 14523-14528. http://dx.doi.org/10.1073/ pnas.96.25.14523

[6] JAGGI M, JOHANSSON SL, BAKER JJ, SMITH LM, GALICH A et al. Aberrant expression of E-cadherin and beta-catenin in human prostate cancer. Urol Oncol 2005; 23: 402-406. http:// dx.doi.org/10.1016/j.urolonc.2005.03.024

[7] OZKAN EE. Plasma and tissue insulin-like growth factor-I receptor (IGF-IR) as a prognostic marker for prostate cancer and anti-IGF-IR agents as novel therapeutic strategy for refractory cases: a review. Mol Cell Endocrinol 2011; 344: 1-24. http://dx.doi.org/10.1016/j.mce.2011.07.002

[8] GENNIGENS C, MENETRIER-CAUX C, DROZ J. InsulinLike Growth Factor (IGF) family and prostate cancer. Crit Rev Oncol Hematol 2006; 58: 124-145. http://dx.doi.org/10.1016/j. critrevonc.2005.10.003

[9] LI J, XU Y-H, LU Y, MA X-P, CHEN P et al. Identifying Differentially Expressed Genes and Small Molecule Drugs for Prostate Cancer by a Bioinformatics Strategy. Asian Pac J Cancer Prev 2013; 14: 5281-5286. http://dx.doi.org/10.7314/ APJCP.2013.14.9.5281

[10] MAHMOUD AM, YANG W, BOSLAND MC. Soy isoflavones and prostate cancer: A review of molecular mechanisms. J 
Steroid Biochem Mol Biol 2014; 140: 116-132. http://dx.doi. org/10.1016/j.jsbmb.2013.12.010

[11] LI Y, AGARWAL P, RAJAGOPALAN D. A global pathway crosstalk network. Bioinformatics 2008; 24: 1442-1447. http:// dx.doi.org/10.1093/bioinformatics/btn200

[12] FRANCESCONI M, REMONDINI D, NERETTI N, SEDIVY JM, COOPER LN et al. Reconstructing networks of pathways via significance analysis of their intersections. BMC Bioinformatics 2008; 9: S9. http://dx.doi.org/10.1186/14712105-9-S4-S9

[13] LEE J, JU J, PARK S, HONG SJ, YOON S. Inhibition of IGF-1 signaling by genistein: modulation of E-cadherin expression and downregulation of $\beta$-catenin signaling in hormone refractory PC-3 prostate cancer cells. Nutr Cancer 2012; 64: 153-162. http://dx.doi.org/10.1080/01635581.2012.630161

[14] MORENO CS. The Sex-determining region Y-box 4 and homeobox $\mathrm{C} 6$ transcriptional networks in prostate cancer progression: crosstalk with the Wnt, Notch, and PI3K pathways. Am J Pathol 2010; 176: 518-527. http://dx.doi.org/10.2353/ ajpath.2010.090657

[15] ARYEE MJ, LIU W, ENGELMANN JC, NUHN P, GUREL $\mathrm{M}$ et al. DNA methylation alterations exhibit intraindividual stability and interindividual heterogeneity in prostate cancer metastases. Sci Transl Med 2013; 5: 169ra110. http://dx.doi. org/10.1126/scitranslmed.3005211

[16] XU A, SUN S. Genomic profiling screens small molecules of metastatic prostate carcinoma. Oncol Lett 2015; 10: 1402-1408. http://dx.doi.org/10.3892/ol.2015.3472

[17] BOLSTAD B. preprocessCore: A collection of pre-processing functions. R package version 2013; 1.

[18] SMYTH GK. Linear models and empirical bayes methods for assessing differential expression in microarray experiments. Stat Appl Genet Mol Biol 2004; 3: Article3.

[19] BENJAMINI Y, DRAI D, ELMER G, KAFKAFI N, GOLANI I. Controlling the false discovery rate in behavior genetics research. Behav Brain Res 2001; 125: 279-284. http://dx.doi. org/10.1016/S0166-4328(01)00297-2

[20] ASHBURNER M, BALL CA, BLAKE JA, BOTSTEIN D, BUTLER $\mathrm{H}$ et al. Gene Ontology: tool for the unification of biology. Nat Genet 2000; 25: 25-29. http://dx.doi.org/10.1038/75556

[21] WANG J-H, ZHAO L-F, LIN P, SU X-R, CHEN S-J et al. GenCLiP 2.0: a web server for functional clustering of genes and construction of molecular networks based on free terms. Bioinformatics 2014; 30: 2534-2536. http://dx.doi.org/10.1093/ bioinformatics/btu241

[22] KANEHISA M, GOTO S. KEGG: kyoto encyclopedia of genes and genomes. Nucleic Acids Res 2000; 28: 27-30. http://dx.doi. org/10.1093/nar/28.1.27

[23] PRASAD TK, GOEL R, KANDASAMY K, KEERTHIKUMAR S, KUMAR S et al. Human protein reference database - 2009 update. Nucleic Acids Res 2009; 37: D767-D772. http://dx.doi. org/10.1093/nar/gkn892

[24] FRANCESCHINI A, SZKLARCZYK D, FRANKILD S, KUHN M, SIMONOVIC M et al. STRING v9.1: proteinprotein interaction networks, with increased coverage and integration. Nucleic Acids Res 2013; 41: D808-815. http:// dx.doi.org/10.1093/nar/gks1094
[25] KOHL M, WIESE S, WARSCHEID B. Cytoscape: Software for Visualization and Analysis of Biological Networks. p. 291-303. In: M. Hamacher, M. Eisenacher and C. Stephan (Eds.), Data Mining in Proteomics. From Standards to Applications., Springer Science+Business Media, LLC, 2011, pp 461. 978-1-60761-986-4. http://dx.doi.org/10.1007/978-160761-987-1_18

[26] NEPUSZ T, YU H, PACCANARO A. Detecting overlapping protein complexes in protein-protein interaction networks. Nat Methods 2012; 9: 471-472. http://dx.doi.org/10.1038/ nmeth.1938

[27] LIU Z-P, WANG Y, ZHANG X-S, CHEN L. Identifying dysfunctional crosstalk of pathways in various regions of Alzheimer's disease brains. BMC Syst Biol 2010; 4: S11. http:// dx.doi.org/10.1186/1752-0509-4-S2-S11

[28] LINTON KD, HAMDY FC. Early diagnosis and surgical management of prostate cancer. Cancer Treat Rev 2003; 29: 151-160. http://dx.doi.org/10.1016/S0305-7372(03)00096-3

[29] URREGO D, TOMCZAK AP, ZAHED F, ST HMER W, PARDO LA. Potassium channels in cell cycle and cell proliferation. Philos Trans R Soc Lond B Biol Sci 2014; 369: 20130094. http://dx.doi.org/10.1098/rstb.2013.0094

[30] HOEIJMAKERS JH. Genome maintenance mechanisms for preventing cancer. Nature 2001; 411: 366-374. http://dx.doi. org/10.1038/35077232

[31] MIMURA S, MASUDA T, MATSUI T, TAKISAWA H. Central role for cdc45 in establishing an initiation complex of DNA replication in Xenopus egg extracts. Genes Cells 2000; 5: 439452. http://dx.doi.org/10.1046/j.1365-2443.2000.00340.x

[32] TERCERO JA, LABIB K, DIFFLEY JF. DNA synthesis at individual replication forks requires the essential initiation factor Cdc45p. EMBO J 2000; 19: 2082-2093. http://dx.doi. org/10.1093/emboj/19.9.2082

[33] LIU P, BARKLEY LR, DAY T, BI X, SLATER DM et al. The Chk1-mediated S-phase checkpoint targets initiation factor Cdc45 via a Cdc25A/Cdk2-independent mechanism. J Biol Chem 2006; 281: 30631-30644. http://dx.doi.org/10.1074/ jbc.M602982200

[34] POLLOK S, BAUERSCHMIDT C, S NGER J, NASHEUER HP, GROSSE F. Human Cdc45 is a proliferation-associated antigen. FEBS J 2007; 274: 3669-3684. http://dx.doi. org/10.1111/j.1742-4658.2007.05900.x

[35] NAKAGAWA T, KOLLMEYER TM, MORLAN BW, ANDERSON SK, BERGSTRALH EJ et al. A tissue biomarker panel predicting systemic progression after PSA recurrence post-definitive prostate cancer therapy. PLoS One 2008; 3: e2318. http://dx.doi.org/10.1371/journal.pone.0002318

[36] KOSARI F, MUNZ JMA, SAVCI-HEIJINK CD, SPIRO C, KLEE EW et al. Identification of prognostic biomarkers for prostate cancer. Clin Cancer Res 2008; 14: 1734-1743. http:// dx.doi.org/10.1158/1078-0432.CCR-07-1494

[37] SCHMITZ J, WATRIN E, L N RT P, MECHTLER K, PETERS $\mathrm{J}-\mathrm{M}$. Sororin is required for stable binding of cohesin to chromatin and for sister chromatid cohesion in interphase. Curr Biol 2007; 17: 630-636. http://dx.doi.org/10.1016/j. cub.2007.02.029 
[38] NGUYEN M-H, KOINUMA J, UEDA K, ITO T, TSUCHIYA $\mathrm{E}$ et al. Phosphorylation and activation of cell division cycle associated 5 by mitogen-activated protein kinase play a crucial role in human lung carcinogenesis. Cancer Res 2010; 70: 5337-5347. http://dx.doi.org/10.1158/0008-5472.CAN-09$\underline{4372}$

[39] SMALL EJ, FRATESI P, REESE DM, STRANG G, LAUS R et al. Immunotherapy of hormone-refractory prostate cancer with antigen-loaded dendritic cells. J Clin Oncol 2000; 18: 3894-3903. http://dx.doi.org/10.1200/jco.2000.18.23.3894

[40] DE PAEPE B, CREUS KK, DE BLEECKER JL. Chemokines in idiopathic inflammatory myopathies. Front Biosci 2007; 13: 2548-2577. http://dx.doi.org/10.2741/2866

[41] HEMBRUFF SL, CHENG N. Chemokine signaling in cancer: Implications on the tumor microenvironment and therapeutic targeting. Cancer Ther 2009; 7: 254.

[42] VINDRIEUX D, ESCOBAR P, LAZENNEC G. Emerging roles of chemokines in prostate cancer. Endocr Relat Cancer 2009; 16: 663-673. http://dx.doi.org/10.1677/ERC-09-0109

[43] LI J, YEN C, LIAW D, PODSYPANINA K, BOSE S et al. PTEN, a putative protein tyrosine phosphatase gene mutated in human brain, breast, and prostate cancer. Science 1997; 275: 1943-1947. http://dx.doi.org/10.1126/ science. 275.5308 .1943

[44] TENG DH, HU R, LIN H, DAVIS T, ILIEV D et al. MMAC1/ PTEN mutations in primary tumor specimens and tumor cell lines. Cancer Research 1997; 57: 5221-5225.

[45] RISINGER JI, HAYES AK, BERCHUCK A, BARRETT JC. PTEN/MMAC1 mutations in endometrial cancers. Cancer Res 1997; 57: 4736-4738.

[46] RASHEED BA, STENZEL TT, MCLENDON RE, PARSONS R, FRIEDMAN AH et al. PTEN gene mutations are seen in high-grade but not in low-grade gliomas. Cancer Res 1997; 57: 4187-4190.

[47] TASHIRO H, BLAZES MS, WU R, CHO KR, BOSE S et al. Mutations in PTEN are frequent in endometrial carcinoma but rare in other common gynecological malignancies. Cancer Res 1997; 57: 3935-3940.

[48] SUZUKI H, FREIJE D, NUSSKERN DR, OKAMI K, CAIRNS $\mathrm{P}$ et al. Interfocal heterogeneity of PTEN/MMAC1 gene alterations in multiple metastatic prostate cancer tissues. Cancer Res 1998; 58: 204-209. 
Supplemental table 1. Gene list in heatmap.

Gene Cluster

TPST2

SH3BGR

P2RY2

AVPI1

LGALS7B

LGALS7

IGJ

CFTR

LMCD1

CTGF

TNC

STAT6

EFS

GLIPR2

SLC20A2

GFOD1

RAB34

GSTP1

ID4

RGN

RARRES2

DZIP1

FGF2

ANKRD35

MAOB

SLC24A3

NR2F1

PDE5A

MEIS2

RASGRP2

PPAPDC 3

SORBS1

KANK2

CBX7

SLIT2

GSN

IGFBP6

DKK3

SLC16A5

SPOCK3

PAGE4

GLIS1

TBC1D1

PLCL1

CFL2

ATP2B4

DPYSL3

DBNDD2 


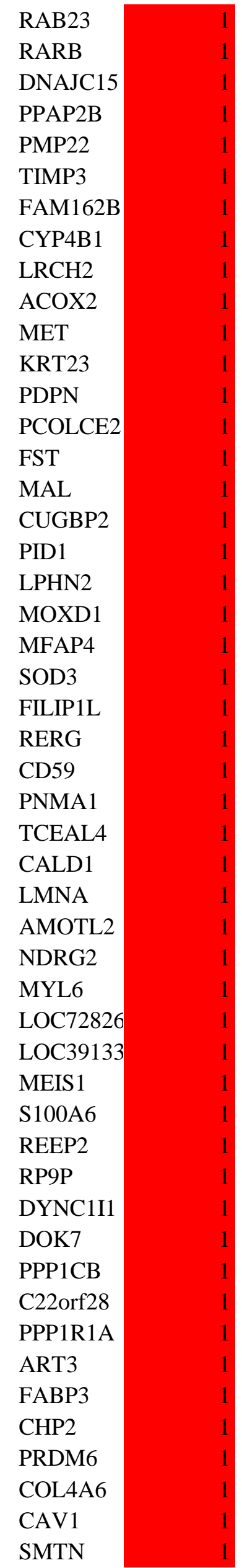




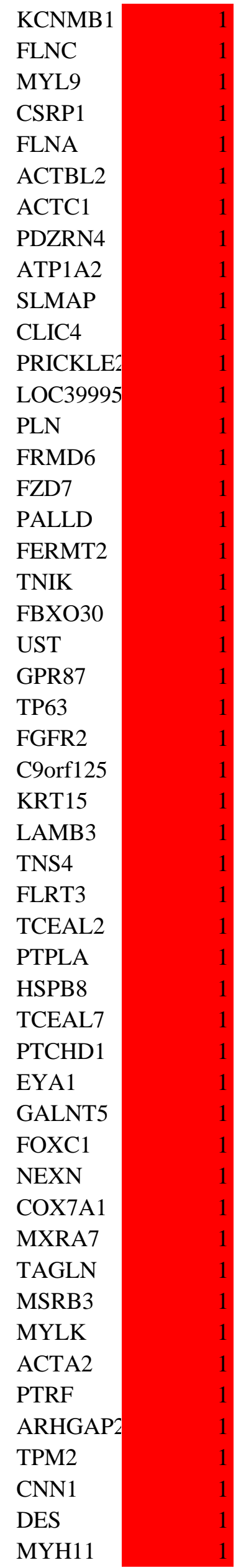




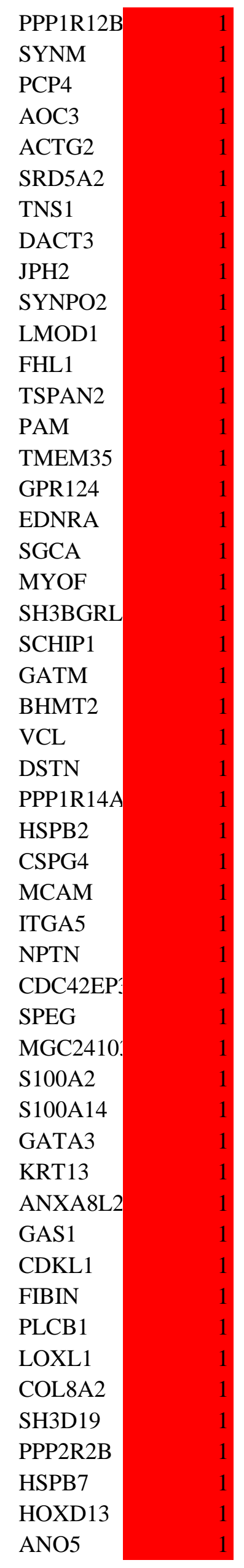




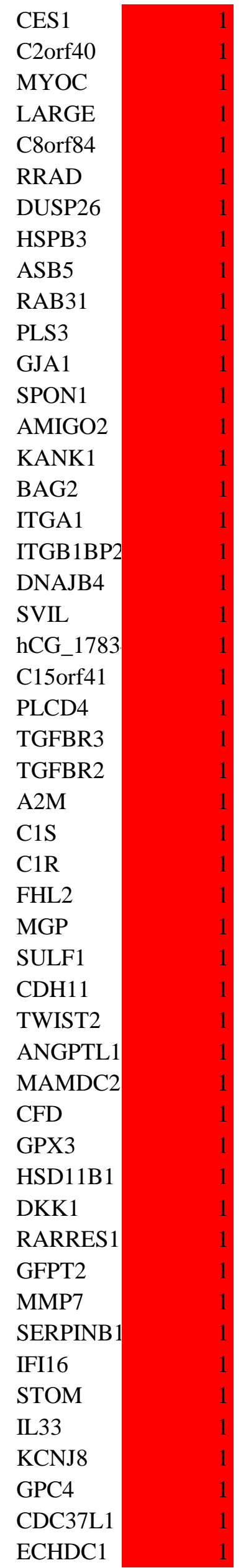




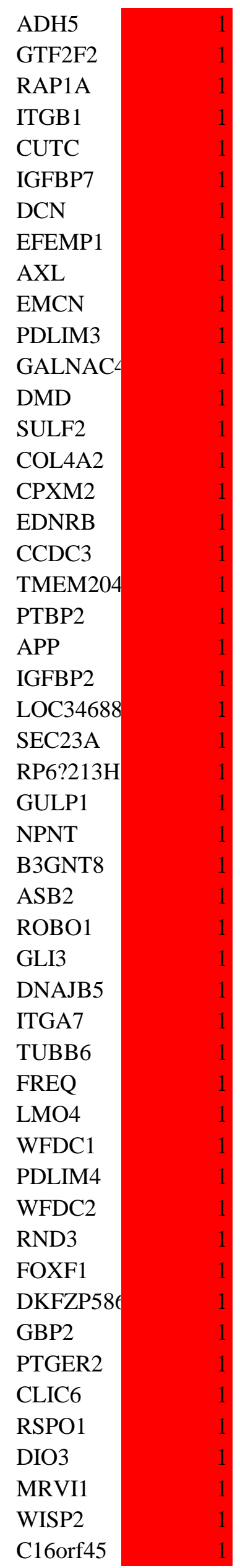




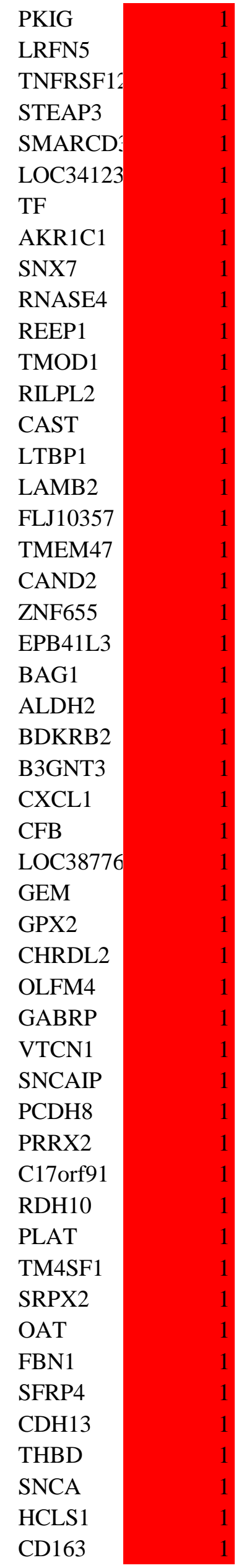




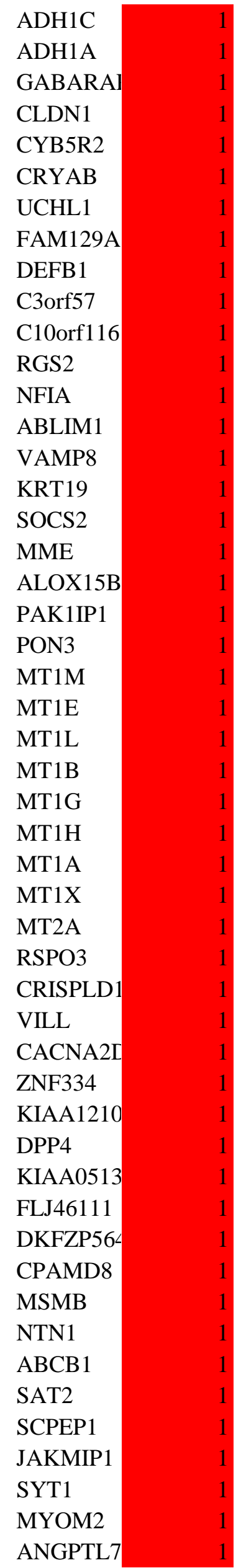




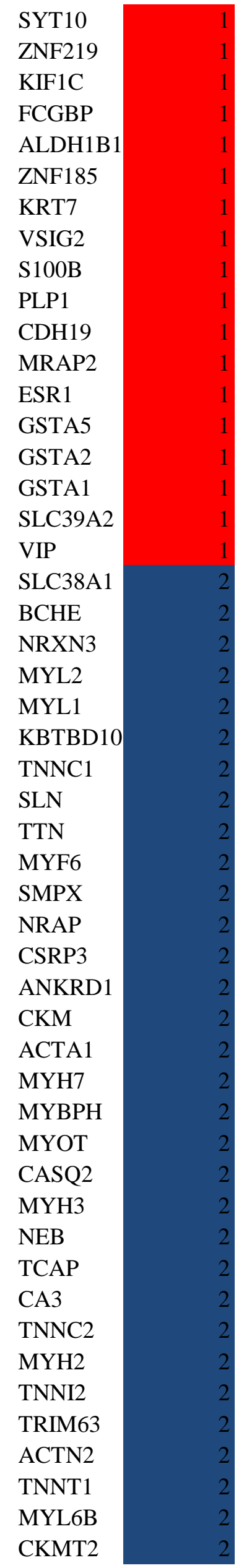




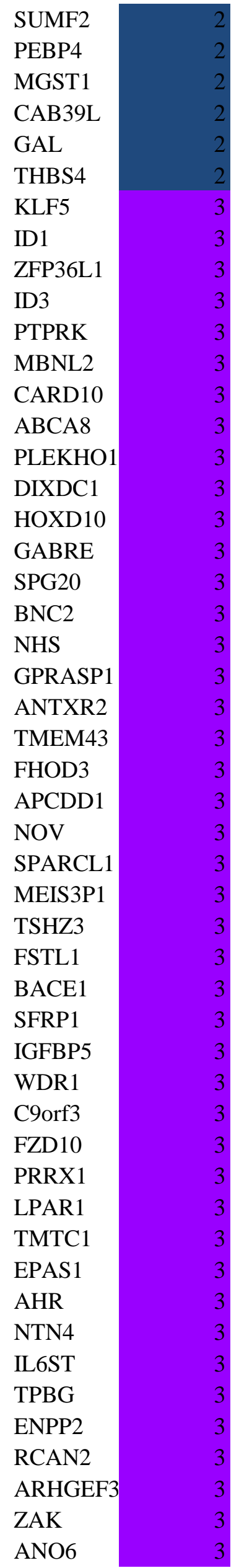




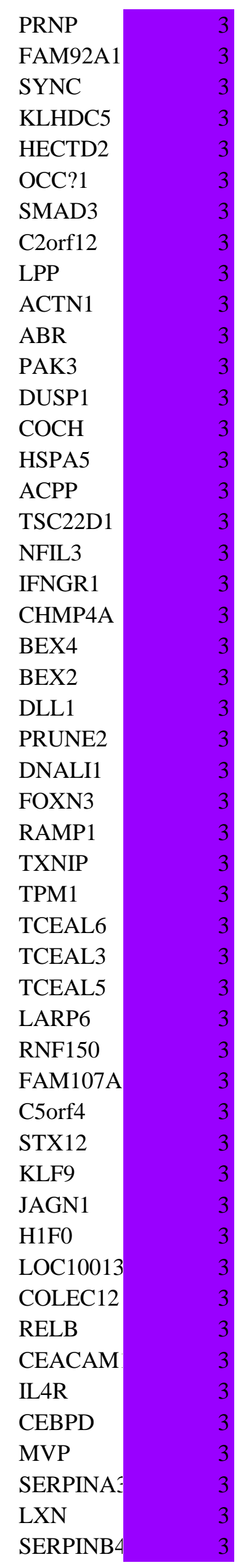




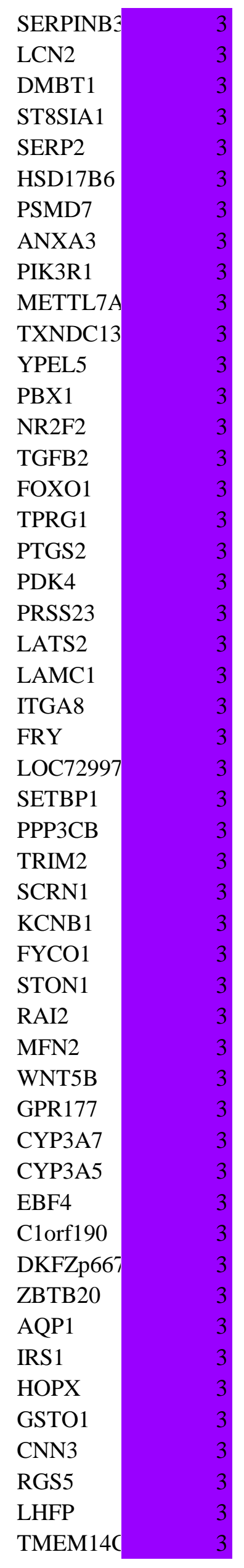




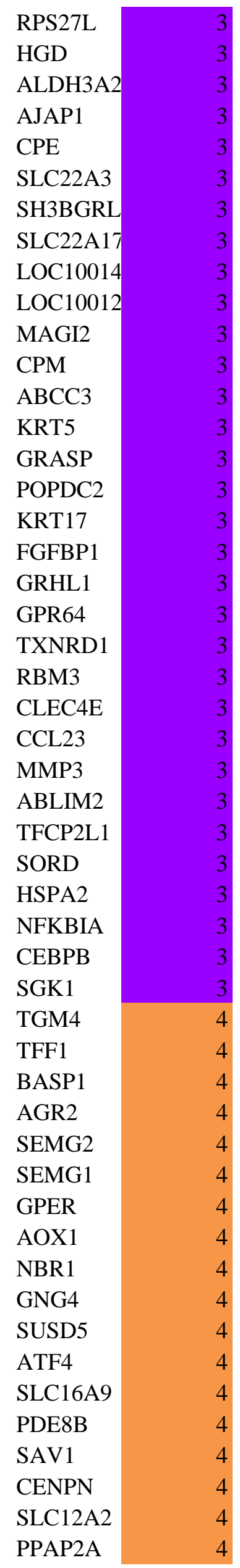




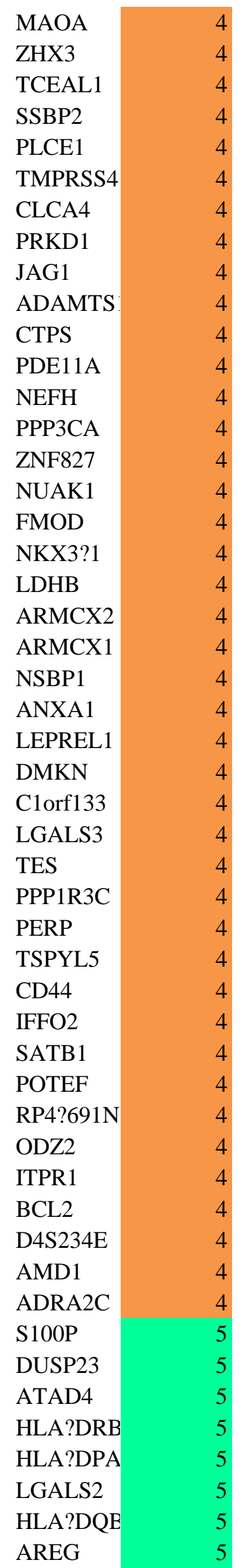




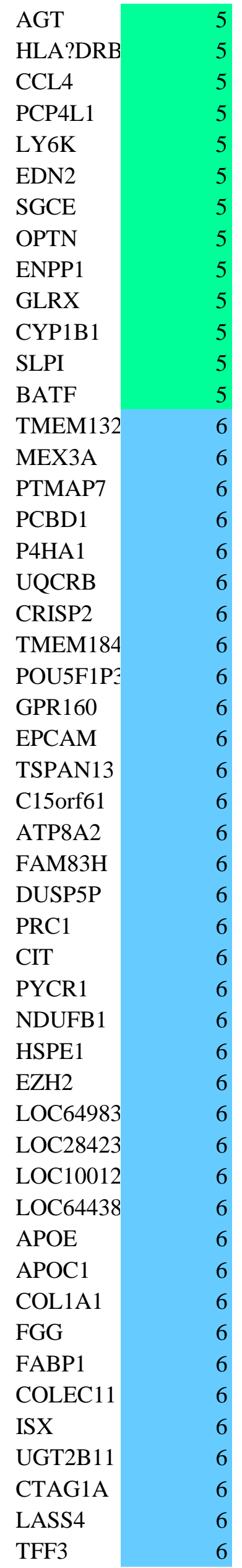




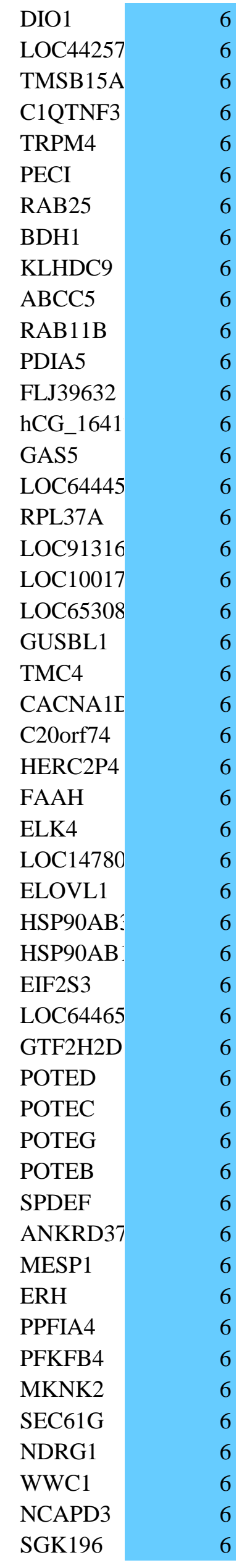




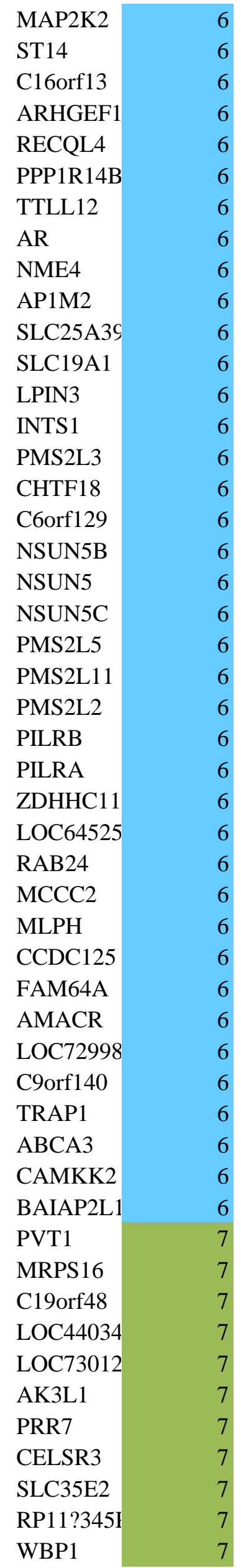




\begin{tabular}{|c|c|}
\hline TDO2 & \\
\hline ACAD8 & \\
\hline HLA?DMF & \\
\hline COL10A1 & \\
\hline KIAA 1683 & \\
\hline KCNQ1OT & \\
\hline GSDMB & \\
\hline GHRLOS & \\
\hline ZNF692 & \\
\hline LOC39949 & \\
\hline СРТ1B & \\
\hline RP11?216I & \\
\hline CPSF1 & \\
\hline SNHG7 & \\
\hline CPNE7 & \\
\hline LOC72841 & \\
\hline LOC10013 & \\
\hline FLJ27365 & \\
\hline LOC85391 & \\
\hline HIST1H4E & \\
\hline RGL2 & \\
\hline RGS11 & \\
\hline B4GALNT & \\
\hline ANKZF1 & \\
\hline SLC45A2 & \\
\hline NBPF20 & \\
\hline HSF4 & \\
\hline ROGDI & \\
\hline ZNF841 & \\
\hline LOC44035 & \\
\hline STAG3L2 & \\
\hline BRD9 & \\
\hline PCDHGB2 & \\
\hline PCDHGA2 & \\
\hline JUN & \\
\hline SOX4 & \\
\hline HSPA6 & \\
\hline TMEM45E & \\
\hline $\mathrm{HPN}$ & \\
\hline DLX1 & \\
\hline APLN & \\
\hline MARCKS] & \\
\hline ESM1 & \\
\hline TMEFF2 & \\
\hline PRIC285 & \\
\hline LOC25578 & \\
\hline CASKIN1 & \\
\hline LOC40135 & \\
\hline LOC43995 & \\
\hline GRIN2D & \\
\hline
\end{tabular}




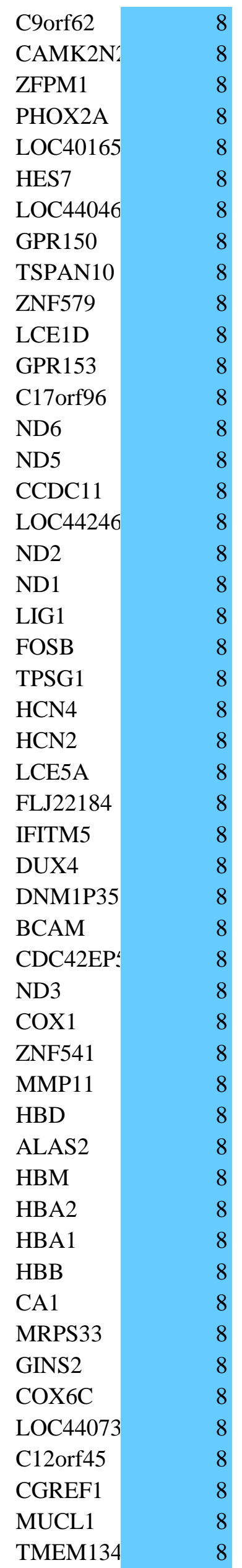




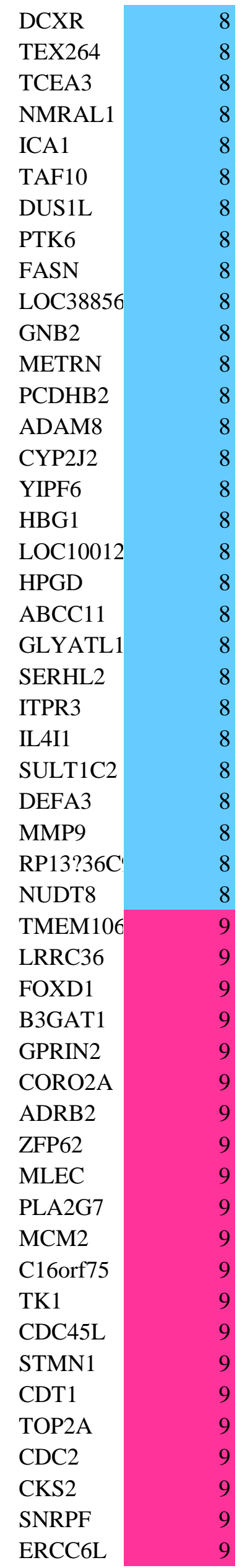




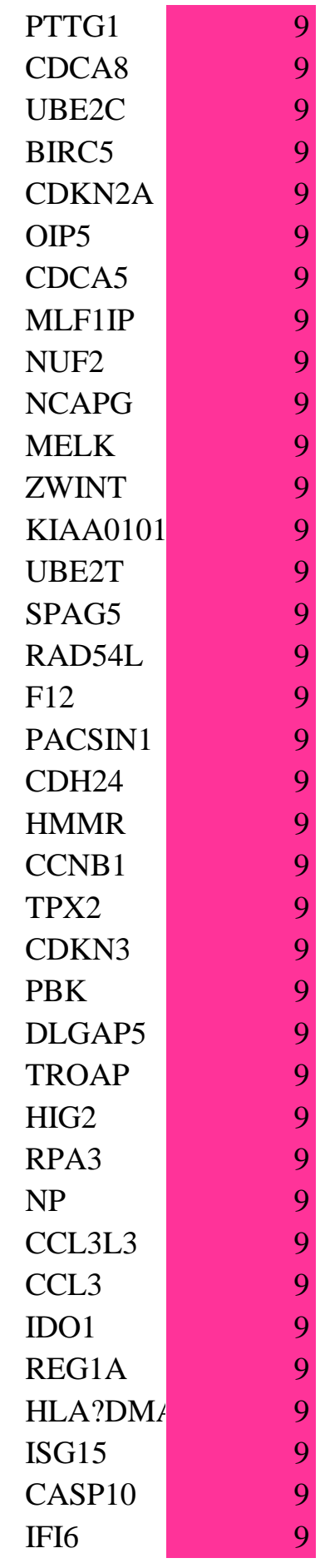


Supplemental table 2. The fold change and p-value of up-regulated and down-regulated differentially expressed genes (DEGs)

\begin{tabular}{|c|c|c|c|c|c|}
\hline Gene id & Gene nam $\epsilon \log F C$ & & P.Value & adj.P.Val & B \\
\hline 56 & AMACR & 3,263 & $9,53 \mathrm{E}-13$ & $3,62 \mathrm{E}-11$ & 18,868 \\
\hline 729 & $\mathrm{HBB}$ & 2,973 & $1,24 \mathrm{E}-08$ & 1,29E-07 & 9,389 \\
\hline 452 & DEFA3 & 2,800 & $1,71 \mathrm{E}-08$ & 1,69E-07 & 9,071 \\
\hline 1045 & LOC73012 & 2,695 & $1,86 \mathrm{E}-06$ & $1,04 \mathrm{E}-05$ & 4,410 \\
\hline 1096 & MCCC2 & 2,676 & $1,25 \mathrm{E}-11$ & $3,16 \mathrm{E}-10$ & 16,290 \\
\hline 14608 & RECQL4 & 2,293 & $1,33 \mathrm{E}-10$ & 2,48E-09 & 13,923 \\
\hline 144 & BIRC5 & 2,193 & 4,04E-09 & $4,80 \mathrm{E}-08$ & 10,513 \\
\hline 960 & LOC10013 & 2,179 & $4,16 \mathrm{E}-12$ & $1,24 \mathrm{E}-10$ & 17,392 \\
\hline $747^{\prime}$ & HIG2 & 2,152 & 7,08E-09 & 7,92E-08 & 9,953 \\
\hline 401 & CRISP2 & 2,150 & $3,88 \mathrm{E}-05$ & $1,57 \mathrm{E}-04$ & 1,420 \\
\hline 1367 & POTEB & 2,128 & $2,44 \mathrm{E}-05$ & 1,04E-04 & 1,877 \\
\hline 84 & $\mathrm{AR}$ & 2,088 & $1,02 \mathrm{E}-08$ & 1,08E-07 & 9,589 \\
\hline $1674^{\prime}$ & STMN1 & 2,071 & $1,84 \mathrm{E}-11$ & $4,41 \mathrm{E}-10$ & 15,903 \\
\hline 1367 & POTEG & 2,032 & $6,47 \mathrm{E}-06$ & $3,16 \mathrm{E}-05$ & 3,180 \\
\hline $540^{\circ}$ & ESM1 & 2,029 & $6,02 \mathrm{E}-17$ & $1,05 \mathrm{E}-14$ & 28,522 \\
\hline 810 & APOE & 2,022 & $1,29 \mathrm{E}-10$ & 2,41E-09 & 13,953 \\
\hline 1191 & ND3 & 2,004 & $8,54 \mathrm{E}-18$ & $2,05 \mathrm{E}-15$ & 30,462 \\
\hline 356 & CHTF18 & 1,949 & $1,08 \mathrm{E}-16$ & $1,71 \mathrm{E}-14$ & 27,937 \\
\hline 1827 & UBE2C & 1,936 & $5,65 \mathrm{E}-10$ & 8,74E-09 & 12,478 \\
\hline 854 & KIAA0101 & 1,910 & $1,19 \mathrm{E}-11$ & $3,03 \mathrm{E}-10$ & 16,344 \\
\hline 345 & CGREF1 & 1,883 & $8,25 \mathrm{E}-09$ & 9,07E-08 & 9,799 \\
\hline 307 & CCL3 & 1,882 & $1,34 \mathrm{E}-17$ & $3,05 \mathrm{E}-15$ & 30,013 \\
\hline 1668 & STAG3L2 & 1,870 & $7,03 \mathrm{E}-14$ & $4,13 \mathrm{E}-12$ & 21,476 \\
\hline 1297 & $\mathrm{PCDHGB}_{2}$ & 1,853 & $8,40 \mathrm{E}-09$ & $9,20 \mathrm{E}-08$ & 9,782 \\
\hline 729 & HBA1 & 1,848 & $1,20 \mathrm{E}-11$ & $3,07 \mathrm{E}-10$ & 16,330 \\
\hline 961 & LOC10017 & 1,847 & $1,24 \mathrm{E}-07$ & $9,52 \mathrm{E}-07$ & 7,097 \\
\hline 1005 & LOC44073 & 1,844 & $1,84 \mathrm{E}-10$ & 3,27E-09 & 13,601 \\
\hline 1031 & LOC6530ع & 1,839 & $2,89 \mathrm{E}-10$ & 4,88E-09 & 13,149 \\
\hline 1036 & LOC72841 & 1,831 & $6,41 \mathrm{E}-13$ & $2,59 \mathrm{E}-11$ & 19,266 \\
\hline 680 & GLYATL1 & 1,791 & $9,68 \mathrm{E}-06$ & $4,52 \mathrm{E}-05$ & 2,783 \\
\hline 729 & HBD & 1,787 & $8,11 \mathrm{E}-07$ & 4,97E-06 & 5,234 \\
\hline 742 & HERC2P4 & 1,782 & $5,69 \mathrm{E}-10$ & 8,78E-09 & 12,472 \\
\hline 1291 & PBK & 1,780 & 2,39E-08 & 2,24E-07 & 8,741 \\
\hline 334 & CDT1 & 1,762 & 5,24E-09 & $6,06 \mathrm{E}-08$ & 10,253 \\
\hline 771 & $\mathrm{HPN}$ & 1,756 & $1,10 \mathrm{E}-13$ & $5,97 \mathrm{E}-12$ & 21,024 \\
\hline 127 & B4GALNT & 1,751 & $6,44 \mathrm{E}-11$ & 1,31E-09 & 14,652 \\
\hline 1367 & POTEC & 1,737 & $3,66 \mathrm{E}-06$ & $1,90 \mathrm{E}-05$ & 3,742 \\
\hline 67 & ANKRD3: & 1,720 & 1,04E-06 & $6,20 \mathrm{E}-06$ & 4,986 \\
\hline 1644 & SPAG5 & 1,718 & $5,69 \mathrm{E}-12$ & $1,64 \mathrm{E}-10$ & 17,079 \\
\hline
\end{tabular}




\begin{tabular}{|c|c|c|c|c|}
\hline 8376 JUN & 1,712 & $3,66 \mathrm{E}-11$ & $8,00 \mathrm{E}-10$ & 15,217 \\
\hline 11862 NCAPG & 1,707 & $1,15 \mathrm{E}-08$ & $1,20 \mathrm{E}-07$ & 9,466 \\
\hline 17366 TK1 & 1,697 & $1,02 \mathrm{E}-05$ & $4,73 \mathrm{E}-05$ & 2,735 \\
\hline 9281 LOC10012 & 1,691 & $4,59 \mathrm{E}-03$ & 1,08E-02 & $-3,170$ \\
\hline 17447 TMEFF2 & 1,688 & $1,35 \mathrm{E}-11$ & $3,36 \mathrm{E}-10$ & 16,217 \\
\hline 10480 LOC85391 & 1,674 & $3,46 \mathrm{E}-06$ & $1,82 \mathrm{E}-05$ & 3,797 \\
\hline 11638 MUCL1 & 1,668 & $5,56 \mathrm{E}-06$ & $2,76 \mathrm{E}-05$ & 3,330 \\
\hline 3953 CPNE7 & 1,655 & $7,18 \mathrm{E}-08$ & 5,91E-07 & 7,643 \\
\hline 11307 MMP11 & 1,644 & $1,91 \mathrm{E}-10$ & 3,39E-09 & 13,562 \\
\hline 14228 PTTG1 & 1,628 & $1,69 \mathrm{E}-09$ & 2,28E-08 & 11,383 \\
\hline 785 APLN & 1,610 & $2,39 \mathrm{E}-15$ & $2,38 \mathrm{E}-13$ & 24,853 \\
\hline 14985 RPA3 & 1,610 & $1,14 \mathrm{E}-13$ & $6,13 \mathrm{E}-12$ & 20,991 \\
\hline 7439 HES7 & 1,607 & $3,93 \mathrm{E}-14$ & $2,56 \mathrm{E}-12$ & 22,059 \\
\hline 18306 UBE2T & 1,605 & $3,46 \mathrm{E}-09$ & $4,21 \mathrm{E}-08$ & 10,667 \\
\hline 11861 NCAPD3 & 1,596 & $1,05 \mathrm{E}-13$ & $5,77 \mathrm{E}-12$ & 21,072 \\
\hline 11273 MLF1IP & 1,593 & $2,56 \mathrm{E}-08$ & 2,38E-07 & 8,671 \\
\hline 6140 FLJ27365 & 1,592 & $3,17 \mathrm{E}-10$ & $5,22 \mathrm{E}-09$ & 13,057 \\
\hline 354 ADRB2 & 1,587 & $1,33 \mathrm{E}-10$ & 2,48E-09 & 13,924 \\
\hline $5482 \mathrm{EZH} 2$ & 1,582 & $8,29 \mathrm{E}-10$ & $1,21 \mathrm{E}-08$ & 12,095 \\
\hline 16479 SPDEF & 1,582 & $5,65 \mathrm{E}-11$ & 1,17E-09 & 14,783 \\
\hline 19125 ZFPM1 & 1,579 & $1,10 \mathrm{E}-14$ & $8,47 \mathrm{E}-13$ & 23,330 \\
\hline 11914 ND1 & 1,573 & $4,27 \mathrm{E}-11$ & $9,16 \mathrm{E}-10$ & 15,064 \\
\hline 12159 NME4 & 1,570 & $6,03 \mathrm{E}-16$ & $7,47 \mathrm{E}-14$ & 26,228 \\
\hline 11100 MEX3A & 1,564 & 2,00E-06 & $1,11 \mathrm{E}-05$ & 4,338 \\
\hline 11915 ND2 & 1,561 & $1,87 \mathrm{E}-12$ & $6,31 \mathrm{E}-11$ & 18,196 \\
\hline 3959 CPSF1 & 1,554 & $1,57 \mathrm{E}-13$ & $7,82 \mathrm{E}-12$ & 20,675 \\
\hline 7225 GUSBL1 & 1,554 & $6,27 \mathrm{E}-10$ & 9,53E-09 & 12,375 \\
\hline 14171 PTK6 & 1,548 & $9,85 \mathrm{E}-09$ & $1,05 \mathrm{E}-07$ & 9,623 \\
\hline 4321 CYP2J2 & 1,548 & $6,85 \mathrm{E}-08$ & $5,66 \mathrm{E}-07$ & 7,689 \\
\hline 5487 F12 & 1,544 & $9,65 \mathrm{E}-08$ & 7,61E-07 & 7,348 \\
\hline 13421 PLA2G7 & 1,542 & $8,05 \mathrm{E}-05$ & $3,01 \mathrm{E}-04$ & 0,707 \\
\hline 15667 SGK196 & 1,537 & $7,93 \mathrm{E}-16$ & $9,27 \mathrm{E}-14$ & 25,955 \\
\hline 4722 DLX1 & 1,528 & $2,67 \mathrm{E}-17$ & $5,55 \mathrm{E}-15$ & 29,329 \\
\hline 19480 ZNF579 & 1,523 & $5,20 \mathrm{E}-12$ & $1,51 \mathrm{E}-10$ & 17,171 \\
\hline 18382 UGT2B11 & 1,518 & 2,38E-03 & $6,06 \mathrm{E}-03$ & $-2,552$ \\
\hline 7925 IFITM5 & 1,517 & $2,33 \mathrm{E}-13$ & $1,09 \mathrm{E}-11$ & 20,279 \\
\hline 19067 ZDHHC11 & 1,505 & $1,65 \mathrm{E}-09$ & 2,23E-08 & 11,405 \\
\hline 9083 LCE5A & 1,505 & $9,31 \mathrm{E}-13$ & $3,54 \mathrm{E}-11$ & 18,892 \\
\hline 2694 CA1 & 1,504 & $2,92 \mathrm{E}-05$ & $1,22 \mathrm{E}-04$ & 1,697 \\
\hline $3258 \mathrm{CDC} 42 \mathrm{EP}$ & 1,503 & $7,90 \mathrm{E}-13$ & $3,09 \mathrm{E}-11$ & 19,056 \\
\hline 3326 CDKN2A & 1,497 & $1,25 \mathrm{E}-13$ & $6,57 \mathrm{E}-12$ & 20,900 \\
\hline 19708 ZWINT & 1,497 & $3,62 \mathrm{E}-09$ & $4,37 \mathrm{E}-08$ & 10,622 \\
\hline 11082 METRN & 1,495 & $2,25 \mathrm{E}-13$ & $1,06 \mathrm{E}-11$ & 20,312 \\
\hline
\end{tabular}




\begin{tabular}{|c|c|c|c|c|}
\hline 15042 RPL37A & 1,485 & $2,39 \mathrm{E}-12$ & $7,71 \mathrm{E}-11$ & 17,950 \\
\hline 13672 POTED & 1,478 & $1,45 \mathrm{E}-03$ & $3,93 \mathrm{E}-03$ & $-2,080$ \\
\hline 5825 FASN & 1,473 & $6,77 \mathrm{E}-06$ & $3,28 \mathrm{E}-05$ & 3,135 \\
\hline 6988 GPR160 & 1,471 & $4,33 \mathrm{E}-08$ & $3,78 \mathrm{E}-07$ & 8,147 \\
\hline 264 ADAM8 & 1,468 & $6,95 \mathrm{E}-07$ & $4,36 \mathrm{E}-06$ & 5,386 \\
\hline 805 APOC1 & 1,462 & $6,03 \mathrm{E}-06$ & 2,97E-05 & 3,250 \\
\hline 12923 PCBD1 & 1,460 & $1,29 \mathrm{E}-16$ & $2,00 \mathrm{E}-14$ & 27,766 \\
\hline 6211 FLJ39632 & 1,453 & $1,72 \mathrm{E}-07$ & $1,26 \mathrm{E}-06$ & 6,773 \\
\hline 14252 PVT1 & 1,452 & $1,91 \mathrm{E}-08$ & $1,86 \mathrm{E}-07$ & 8,960 \\
\hline 3898 COX1 & 1,449 & $1,61 \mathrm{E}-11$ & $3,93 \mathrm{E}-10$ & 16,041 \\
\hline 10049 LOC44046 & 1,436 & $1,60 \mathrm{E}-11$ & $3,91 \mathrm{E}-10$ & 16,047 \\
\hline 7585 HLA-DRB & 1,433 & 8,63E-09 & $9,43 \mathrm{E}-08$ & 9,755 \\
\hline 4636 DIO1 & 1,433 & 2,94E-05 & $1,23 \mathrm{E}-04$ & 1,691 \\
\hline $17241 \mathrm{TFF} 3$ & 1,427 & $7,64 \mathrm{E}-05$ & $2,87 \mathrm{E}-04$ & 0,758 \\
\hline 9070 LCE1D & 1,423 & $8,98 \mathrm{E}-12$ & $2,40 \mathrm{E}-10$ & 16,624 \\
\hline 10202 LOC64445 & 1,421 & $5,52 \mathrm{E}-06$ & $2,74 \mathrm{E}-05$ & 3,338 \\
\hline 6307 FOSB & 1,420 & $6,65 \mathrm{E}-22$ & $5,25 \mathrm{E}-19$ & 39,783 \\
\hline 6982 GPR150 & 1,416 & $7,09 \mathrm{E}-13$ & $2,84 \mathrm{E}-11$ & 19,164 \\
\hline 7788 HSP90AB & 1,415 & $2,09 \mathrm{E}-18$ & $6,38 \mathrm{E}-16$ & 31,858 \\
\hline 17838 TPSG1 & 1,413 & $2,06 \mathrm{E}-12$ & $6,82 \mathrm{E}-11$ & 18,096 \\
\hline 1256 B3GAT1 & 1,412 & $4,83 \mathrm{E}-05$ & $1,91 \mathrm{E}-04$ & 1,207 \\
\hline 4130 CTAG1A & 1,412 & $9,79 \mathrm{E}-03$ & $2,10 \mathrm{E}-02$ & $-3,874$ \\
\hline 10526 LPIN3 & 1,405 & $2,80 \mathrm{E}-11$ & $6,31 \mathrm{E}-10$ & 15,487 \\
\hline 9310 LOC10012 & 1,403 & $1,74 \mathrm{E}-13$ & $8,52 \mathrm{E}-12$ & 20,570 \\
\hline 7139 GSDMB & 1,398 & $4,02 \mathrm{E}-07$ & 2,69E-06 & 5,930 \\
\hline 5748 FAM64A & 1,398 & 5,39E-09 & $6,23 \mathrm{E}-08$ & 10,225 \\
\hline 7054 GPRIN2 & 1,396 & $7,19 \mathrm{E}-05$ & $2,72 \mathrm{E}-04$ & 0,818 \\
\hline 4959 DUS1L & 1,393 & 2,22E-09 & $2,87 \mathrm{E}-08$ & 11,111 \\
\hline 4825 DNM1P35 & 1,392 & $2,38 \mathrm{E}-12$ & $7,71 \mathrm{E}-11$ & 17,951 \\
\hline 3606 CKS2 & 1,387 & $1,55 \mathrm{E}-06$ & $8,85 \mathrm{E}-06$ & 4,593 \\
\hline 7293 HBA2 & 1,387 & $7,37 \mathrm{E}-11$ & $1,47 \mathrm{E}-09$ & 14,516 \\
\hline 12413 NUF2 & 1,382 & $1,14 \mathrm{E}-08$ & $1,20 \mathrm{E}-07$ & 9,475 \\
\hline 3233 CDC2 & 1,382 & 2,54E-09 & $3,23 \mathrm{E}-08$ & 10,976 \\
\hline 6983 GPR153 & 1,378 & $1,80 \mathrm{E}-11$ & $4,32 \mathrm{E}-10$ & 15,927 \\
\hline 7915 IFI6 & 1,375 & $1,05 \mathrm{E}-07$ & $8,18 \mathrm{E}-07$ & 7,268 \\
\hline 7622 HMMR & 1,372 & $7,86 \mathrm{E}-07$ & $4,84 \mathrm{E}-06$ & 5,264 \\
\hline 2670 C9orf62 & 1,371 & $3,09 \mathrm{E}-14$ & $2,10 \mathrm{E}-12$ & 22,298 \\
\hline 3590 CIT & 1,368 & $1,35 \mathrm{E}-13$ & $6,99 \mathrm{E}-12$ & 20,823 \\
\hline 12511 OIP5 & 1,368 & $4,12 \mathrm{E}-11$ & $8,92 \mathrm{E}-10$ & 15,097 \\
\hline 5320 ЕРСАМ & 1,364 & $2,64 \mathrm{E}-10$ & 4,51E-09 & 13,238 \\
\hline 1869 C16orf13 & 1,363 & $1,02 \mathrm{E}-15$ & $1,15 \mathrm{E}-13$ & 25,706 \\
\hline 3270 CDCA5 & 1,352 & $9,18 \mathrm{E}-09$ & $9,92 \mathrm{E}-08$ & 9,694 \\
\hline 1192 АTP8A2 & 1,351 & $9,57 \mathrm{E}-06$ & $4,48 \mathrm{E}-05$ & 2,795 \\
\hline
\end{tabular}




\begin{tabular}{|c|c|c|c|c|}
\hline $5384 \mathrm{ERH}$ & 1,351 & $8,18 \mathrm{E}-13$ & $3,18 \mathrm{E}-11$ & 19,021 \\
\hline 14301 RAB11B & 1,350 & $1,37 \mathrm{E}-03$ & $3,74 \mathrm{E}-03$ & $-2,029$ \\
\hline 14266 PYCR1 & 1,346 & $8,20 \mathrm{E}-13$ & $3,18 \mathrm{E}-11$ & 19,019 \\
\hline 10009 LOC40165 & 1,345 & $8,99 \mathrm{E}-11$ & $1,76 \mathrm{E}-09$ & 14,318 \\
\hline 2791 CAMKK2 & 1,343 & $1,27 \mathrm{E}-10$ & 2,38E-09 & 13,971 \\
\hline 14962 RP13-36C! & 1,339 & $1,26 \mathrm{E}-02$ & 2,61E-02 & $-4,104$ \\
\hline 11918 ND6 & 1,331 & $2,30 \mathrm{E}-12$ & $7,50 \mathrm{E}-11$ & 17,987 \\
\hline $7300 \mathrm{HBM}$ & 1,329 & $2,20 \mathrm{E}-05$ & $9,43 \mathrm{E}-05$ & 1,978 \\
\hline 66 ABCC11 & 1,323 & $3,29 \mathrm{E}-03$ & $8,08 \mathrm{E}-03$ & $-2,858$ \\
\hline 2726 CACNA1I & 1,322 & $1,44 \mathrm{E}-06$ & $8,29 \mathrm{E}-06$ & 4,667 \\
\hline 13851 PRC1 & 1,320 & $4,05 \mathrm{E}-12$ & $1,21 \mathrm{E}-10$ & 17,420 \\
\hline $13271 \mathrm{PHOX} 2 \mathrm{~A}$ & 1,313 & $2,95 \mathrm{E}-12$ & $9,23 \mathrm{E}-11$ & 17,737 \\
\hline 7363 HCN2 & 1,310 & $2,22 \mathrm{E}-11$ & $5,21 \mathrm{E}-10$ & 15,717 \\
\hline 6118 FLJ22184 & 1,303 & $1,03 \mathrm{E}-12$ & $3,86 \mathrm{E}-11$ & 18,794 \\
\hline 17594 TMEM45I & 1,300 & $1,54 \mathrm{E}-03$ & $4,16 \mathrm{E}-03$ & $-2,142$ \\
\hline 10443 LOC72998 & 1,296 & $3,60 \mathrm{E}-10$ & $5,83 \mathrm{E}-09$ & 12,929 \\
\hline 10839 MAP2K2 & 1,291 & $2,35 \mathrm{E}-13$ & $1,10 \mathrm{E}-11$ & 20,268 \\
\hline 2477 C6orf129 & 1,285 & $8,31 \mathrm{E}-14$ & $4,72 \mathrm{E}-12$ & 21,308 \\
\hline 11327 MMP9 & 1,283 & $2,14 \mathrm{E}-04$ & $7,18 \mathrm{E}-04$ & $-0,241$ \\
\hline 5502 FAAH & 1,282 & $8,65 \mathrm{E}-09$ & $9,44 \mathrm{E}-08$ & 9,753 \\
\hline 2636 C9orf140 & 1,279 & $9,07 \mathrm{E}-11$ & $1,77 \mathrm{E}-09$ & 14,309 \\
\hline 3262 CDC45L & 1,278 & 4,91E-08 & $4,23 \mathrm{E}-07$ & 8,020 \\
\hline 18001 TRPM4 & 1,272 & $3,99 \mathrm{E}-08$ & $3,52 \mathrm{E}-07$ & 8,227 \\
\hline 16649 ST14 & 1,272 & $1,70 \mathrm{E}-10$ & $3,05 \mathrm{E}-09$ & 13,681 \\
\hline 7299 HBG1 & 1,271 & $1,28 \mathrm{E}-04$ & $4,56 \mathrm{E}-04$ & 0,256 \\
\hline 17420 TMC4 & 1,262 & $1,11 \mathrm{E}-11$ & $2,86 \mathrm{E}-10$ & 16,413 \\
\hline 2945 CCDC125 & 1,261 & $3,92 \mathrm{E}-10$ & 6,29E-09 & 12,843 \\
\hline 14703 RGS11 & 1,258 & $1,34 \mathrm{E}-12$ & $4,81 \mathrm{E}-11$ & 18,528 \\
\hline 9661 LOC1478C & 1,257 & $2,56 \mathrm{E}-09$ & $3,25 \mathrm{E}-08$ & 10,968 \\
\hline 14928 ROGDI & 1,255 & $3,80 \mathrm{E}-10$ & $6,12 \mathrm{E}-09$ & 12,875 \\
\hline 492 ALAS2 & 1,252 & 2,38E-07 & $1,68 \mathrm{E}-06$ & 6,452 \\
\hline 12345 NSUN5B & 1,250 & $2,27 \mathrm{E}-14$ & $1,60 \mathrm{E}-12$ & 22,604 \\
\hline 3847 COLEC11 & 1,243 & $3,68 \mathrm{E}-05$ & $1,49 \mathrm{E}-04$ & 1,472 \\
\hline 743 AP1M2 & 1,243 & $1,56 \mathrm{E}-10$ & 2,84E-09 & 13,764 \\
\hline 3377 CELSR3 & 1,243 & $1,30 \mathrm{E}-09$ & $1,81 \mathrm{E}-08$ & 11,643 \\
\hline 7715 HPGD & 1,243 & $3,61 \mathrm{E}-03$ & $8,75 \mathrm{E}-03$ & $-2,945$ \\
\hline 10026 LOC43995 & 1,239 & $1,21 \mathrm{E}-12$ & $4,42 \mathrm{E}-11$ & 18,632 \\
\hline 11917 ND5 & 1,234 & $9,97 \mathrm{E}-14$ & $5,49 \mathrm{E}-12$ & 21,126 \\
\hline 13770 PPP1R14E & 1,234 & $5,76 \mathrm{E}-09$ & $6,60 \mathrm{E}-08$ & 10,158 \\
\hline 17671 TMSB15A & 1,230 & 2,98E-04 & $9,63 \mathrm{E}-04$ & $-0,563$ \\
\hline 3332 CDKN3 & 1,229 & $1,82 \mathrm{E}-08$ & $1,78 \mathrm{E}-07$ & 9,010 \\
\hline 12344 NSUN5 & 1,229 & $7,71 \mathrm{E}-17$ & $1,28 \mathrm{E}-14$ & 28,276 \\
\hline 13725 PPFIA4 & 1,227 & $3,71 \mathrm{E}-06$ & $1,93 \mathrm{E}-05$ & 3,729 \\
\hline
\end{tabular}




\begin{tabular}{|c|c|c|c|c|}
\hline 14319 RAB24 & 1,227 & $9,30 \mathrm{E}-15$ & $7,39 \mathrm{E}-13$ & 23,498 \\
\hline 17219 TEX264 & 1,225 & $2,70 \mathrm{E}-12$ & $8,54 \mathrm{E}-11$ & 17,828 \\
\hline 3273 CDCA8 & 1,220 & $9,25 \mathrm{E}-13$ & $3,53 \mathrm{E}-11$ & 18,898 \\
\hline 13339 PILRB & 1,217 & $1,46 \mathrm{E}-10$ & $2,68 \mathrm{E}-09$ & 13,829 \\
\hline 4990 DUX4 & 1,215 & $3,12 \mathrm{E}-10$ & $5,17 \mathrm{E}-09$ & 13,074 \\
\hline 14616 REG1A & 1,214 & $7,34 \mathrm{E}-03$ & $1,63 \mathrm{E}-02$ & $-3,608$ \\
\hline 11485 MRPS16 & 1,214 & $2,04 \mathrm{E}-18$ & $6,38 \mathrm{E}-16$ & 31,884 \\
\hline 17987 TROAP & 1,213 & 3,53E-09 & $4,27 \mathrm{E}-08$ & 10,648 \\
\hline 2162 C1QTNF3 & 1,212 & $5,00 \mathrm{E}-13$ & $2,10 \mathrm{E}-11$ & 19,515 \\
\hline 4714 DLGAP5 & 1,212 & $4,39 \mathrm{E}-08$ & $3,83 \mathrm{E}-07$ & 8,132 \\
\hline 15877 SLC19A1 & 1,211 & $2,79 \mathrm{E}-15$ & $2,70 \mathrm{E}-13$ & 24,700 \\
\hline 7571 HLA-DM & 1,210 & $1,81 \mathrm{E}-08$ & $1,77 \mathrm{E}-07$ & 9,016 \\
\hline 7365 HCN4 & 1,208 & $1,39 \mathrm{E}-10$ & $2,56 \mathrm{E}-09$ & 13,881 \\
\hline 8489 KCNQ1O'] & 1,207 & $1,17 \mathrm{E}-06$ & $6,92 \mathrm{E}-06$ & 4,868 \\
\hline 5779 FAM83H & 1,206 & $2,50 \mathrm{E}-12$ & $8,02 \mathrm{E}-11$ & 17,905 \\
\hline 7801 HSPA6 & 1,205 & $1,65 \mathrm{E}-04$ & $5,71 \mathrm{E}-04$ & 0,008 \\
\hline 127 ACAD8 & 1,205 & $2,71 \mathrm{E}-08$ & $2,50 \mathrm{E}-07$ & 8,614 \\
\hline 11076 MESP1 & 1,202 & $2,96 \mathrm{E}-06$ & $1,57 \mathrm{E}-05$ & 3,953 \\
\hline 13553 PMS2L5 & 1,200 & $2,22 \mathrm{E}-15$ & $2,27 \mathrm{E}-13$ & 24,927 \\
\hline 14688 RGL2 & 1,200 & $2,11 \mathrm{E}-12$ & $6,98 \mathrm{E}-11$ & 18,071 \\
\hline 12779 P4HA1 & 1,199 & $3,12 \mathrm{E}-09$ & $3,85 \mathrm{E}-08$ & 10,771 \\
\hline 3085 CCNB1 & 1,195 & $2,53 \mathrm{E}-08$ & $2,36 \mathrm{E}-07$ & 8,684 \\
\hline 3814 COL1A1 & 1,193 & $8,08 \mathrm{E}-08$ & $6,52 \mathrm{E}-07$ & 7,525 \\
\hline 14401 RAD54L & 1,192 & $4,11 \mathrm{E}-09$ & $4,87 \mathrm{E}-08$ & 10,496 \\
\hline 3966 СРТ1B & 1,191 & $1,43 \mathrm{E}-07$ & $1,07 \mathrm{E}-06$ & 6,957 \\
\hline 1075 ATAD4 & 1,191 & $1,37 \mathrm{E}-03$ & $3,73 \mathrm{E}-03$ & $-2,025$ \\
\hline 72 ABCC5 & 1,186 & $6,81 \mathrm{E}-08$ & $5,64 \mathrm{E}-07$ & 7,696 \\
\hline 17455 TMEM106 & 1,184 & $6,94 \mathrm{E}-07$ & $4,35 \mathrm{E}-06$ & 5,388 \\
\hline 8676 KIAA168? & 1,182 & $2,88 \mathrm{E}-05$ & $1,20 \mathrm{E}-04$ & 1,712 \\
\hline 1302 BAIAP2L] & 1,180 & $8,15 \mathrm{E}-13$ & $3,17 \mathrm{E}-11$ & 19,025 \\
\hline 13889 PRIC285 & 1,180 & $2,29 \mathrm{E}-12$ & $7,50 \mathrm{E}-11$ & 17,990 \\
\hline 16411 SOX4 & 1,178 & $4,45 \mathrm{E}-07$ & 2,94E-06 & 5,829 \\
\hline 13338 PILRA & 1,175 & $1,06 \mathrm{E}-14$ & $8,25 \mathrm{E}-13$ & 23,364 \\
\hline 15533 SERHL2 & 1,173 & $3,10 \mathrm{E}-03$ & $7,65 \mathrm{E}-03$ & $-2,801$ \\
\hline 2011 C19orf48 & 1,171 & $4,64 \mathrm{E}-05$ & $1,84 \mathrm{E}-04$ & 1,245 \\
\hline 1897 C16orf75 & 1,169 & $1,45 \mathrm{E}-06$ & $8,37 \mathrm{E}-06$ & 4,656 \\
\hline 10198 LOC64438 & 1,169 & $3,18 \mathrm{E}-08$ & 2,89E-07 & 8,454 \\
\hline 18473 UQCRB & 1,168 & $1,78 \mathrm{E}-11$ & $4,30 \mathrm{E}-10$ & 15,937 \\
\hline 2851 CASKIN1 & 1,165 & $1,61 \mathrm{E}-13$ & $8,00 \mathrm{E}-12$ & 20,647 \\
\hline 13550 PMS2L11 & 1,165 & $1,35 \mathrm{E}-15$ & $1,47 \mathrm{E}-13$ & 25,423 \\
\hline 10222 LOC64525 & 1,159 & $2,05 \mathrm{E}-13$ & $9,75 \mathrm{E}-12$ & 20,408 \\
\hline 17845 TPX2 & 1,156 & $3,37 \mathrm{E}-10$ & $5,50 \mathrm{E}-09$ & 12,997 \\
\hline 6008 FGG & 1,155 & $2,20 \mathrm{E}-03$ & $5,66 \mathrm{E}-03$ & $-2,478$ \\
\hline
\end{tabular}




\begin{tabular}{|c|c|c|c|c|}
\hline 5219 ELK4 & 1,154 & $3,22 \mathrm{E}-15$ & $3,00 \mathrm{E}-13$ & 24,557 \\
\hline 9145 LGALS2 & 1,153 & 2,07E-07 & 1,49E-06 & 6,589 \\
\hline 10488 LOC91316 & 1,152 & $1,07 \mathrm{E}-10$ & 2,04E-09 & 14,148 \\
\hline 19651 ZNF841 & 1,151 & $2,37 \mathrm{E}-08$ & 2,23E-07 & 8,747 \\
\hline 4450 DCXR & 1,151 & $1,57 \mathrm{E}-05$ & $6,98 \mathrm{E}-05$ & 2,305 \\
\hline 18953 YIPF6 & 1,150 & $1,83 \mathrm{E}-05$ & 7,99E-05 & 2,158 \\
\hline 10904 MARCKS & 1,149 & $4,55 \mathrm{E}-15$ & $3,97 \mathrm{E}-13$ & 24,212 \\
\hline 3803 COL10A1 & 1,143 & $2,60 \mathrm{E}-04$ & $8,53 \mathrm{E}-04$ & $-0,431$ \\
\hline 17779 TOP2A & 1,140 & $5,00 \mathrm{E}-07$ & $3,25 \mathrm{E}-06$ & 5,713 \\
\hline 19443 ZNF541 & 1,138 & $8,75 \mathrm{E}-06$ & $4,13 \mathrm{E}-05$ & 2,883 \\
\hline 18716 WBP1 & 1,136 & $6,52 \mathrm{E}-11$ & $1,32 \mathrm{E}-09$ & 14,639 \\
\hline 16822 SUMF2 & 1,135 & $4,54 \mathrm{E}-11$ & $9,69 \mathrm{E}-10$ & 15,001 \\
\hline 1866 C15orf61 & 1,134 & $1,02 \mathrm{E}-15$ & $1,15 \mathrm{E}-13$ & 25,701 \\
\hline 4982 DUSP5P & 1,131 & $3,26 \mathrm{E}-10$ & $5,35 \mathrm{E}-09$ & 13,029 \\
\hline 17867 TRAP1 & 1,131 & $3,49 \mathrm{E}-17$ & $6,88 \mathrm{E}-15$ & 29,065 \\
\hline 7896 IDO1 & 1,131 & 2,91E-07 & $2,02 \mathrm{E}-06$ & 6,251 \\
\hline 13178 PFKFB4 & 1,130 & $1,20 \mathrm{E}-09$ & $1,68 \mathrm{E}-08$ & 11,725 \\
\hline 18135 TTLL12 & 1,128 & $1,44 \mathrm{E}-07$ & $1,08 \mathrm{E}-06$ & 6,947 \\
\hline 1955 C17orf96 & 1,125 & 9,11E-09 & $9,87 \mathrm{E}-08$ & 9,700 \\
\hline 5167 EIF2S3 & 1,124 & $1,28 \mathrm{E}-11$ & $3,21 \mathrm{E}-10$ & 16,270 \\
\hline 7584 HLA-DRB & 1,122 & $1,15 \mathrm{E}-07$ & $8,88 \mathrm{E}-07$ & 7,175 \\
\hline 16815 SULT1C2 & 1,121 & $1,15 \mathrm{E}-04$ & $4,15 \mathrm{E}-04$ & 0,356 \\
\hline 17535 TMEM184 & 1,119 & $3,21 \mathrm{E}-05$ & $1,32 \mathrm{E}-04$ & 1,607 \\
\hline 7821 HSPE1 & 1,119 & $8,55 \mathrm{E}-11$ & $1,68 \mathrm{E}-09$ & 14,368 \\
\hline 16299 SNHG7 & 1,117 & 2,74E-08 & $2,52 \mathrm{E}-07$ & 8,601 \\
\hline 5231 ELOVL1 & 1,116 & $1,10 \mathrm{E}-13$ & $5,97 \mathrm{E}-12$ & 21,024 \\
\hline 13689 POU5F1P: & 1,116 & $9,19 \mathrm{E}-07$ & $5,55 \mathrm{E}-06$ & 5,110 \\
\hline 6845 GNB2 & 1,114 & $1,03 \mathrm{E}-14$ & $8,09 \mathrm{E}-13$ & 23,396 \\
\hline 10980 MCM2 & 1,114 & $5,51 \mathrm{E}-08$ & 4,67E-07 & 7,906 \\
\hline 8173 INTS1 & 1,113 & $5,16 \mathrm{E}-14$ & $3,21 \mathrm{E}-12$ & 21,785 \\
\hline 5496 F5 & 1,113 & $1,40 \mathrm{E}-02$ & $2,86 \mathrm{E}-02$ & $-4,202$ \\
\hline 10209 LOC64465 & 1,112 & $1,59 \mathrm{E}-08$ & $1,59 \mathrm{E}-07$ & 9,143 \\
\hline 12964 PCDHB2 & 1,110 & $2,44 \mathrm{E}-05$ & $1,04 \mathrm{E}-04$ & 1,874 \\
\hline 7107 GRIN2D & 1,110 & $1,78 \mathrm{E}-11$ & $4,30 \mathrm{E}-10$ & 15,937 \\
\hline 12410 NUDT8 & 1,110 & $5,08 \mathrm{E}-05$ & $2,00 \mathrm{E}-04$ & 1,157 \\
\hline 7311 hCG_1641 & 1,109 & $1,86 \mathrm{E}-13$ & $9,02 \mathrm{E}-12$ & 20,505 \\
\hline 13129 PEBP4 & 1,107 & $1,76 \mathrm{E}-05$ & $7,74 \mathrm{E}-05$ & 2,193 \\
\hline 3078 CCL4 & 1,106 & $1,30 \mathrm{E}-06$ & 7,61E-06 & 4,763 \\
\hline 17484 TMEM134 & 1,105 & $6,16 \mathrm{E}-09$ & $7,00 \mathrm{E}-08$ & 10,091 \\
\hline 7581 HLA-DQB & 1,103 & $5,28 \mathrm{E}-06$ & $2,64 \mathrm{E}-05$ & 3,381 \\
\hline 12972 PCDHGA & 1,103 & $5,93 \mathrm{E}-11$ & $1,22 \mathrm{E}-09$ & 14,735 \\
\hline $3291 \mathrm{CDH} 24$ & 1,102 & $1,88 \mathrm{E}-13$ & $9,06 \mathrm{E}-12$ & 20,495 \\
\hline 6718 GINS2 & 1,100 & 3,71E-07 & 2,51E-06 & 6,010 \\
\hline
\end{tabular}




\begin{tabular}{|c|c|c|c|c|}
\hline 13131 PECI & 1,096 & $6,09 \mathrm{E}-07$ & $3,88 \mathrm{E}-06$ & 5,517 \\
\hline 2930 CCDC11 & 1,096 & $1,82 \mathrm{E}-11$ & $4,36 \mathrm{E}-10$ & 15,916 \\
\hline 6704 GHRLOS & 1,093 & 3,01E-09 & $3,74 \mathrm{E}-08$ & 10,806 \\
\hline 16943 TAF10 & 1,090 & $2,67 \mathrm{E}-11$ & $6,06 \mathrm{E}-10$ & 15,532 \\
\hline 9049 LASS4 & 1,090 & $9,68 \mathrm{E}-10$ & $1,39 \mathrm{E}-08$ & 11,940 \\
\hline 13552 PMS2L3 & 1,088 & $6,93 \mathrm{E}-18$ & $1,75 \mathrm{E}-15$ & 30,669 \\
\hline 9959 LOC3994C & 1,087 & $4,42 \mathrm{E}-07$ & 2,92E-06 & 5,837 \\
\hline 12801 PACSIN1 & 1,082 & $4,18 \mathrm{E}-09$ & $4,94 \mathrm{E}-08$ & 10,479 \\
\hline 6316 FOXD1 & 1,082 & $1,08 \mathrm{E}-08$ & $1,14 \mathrm{E}-07$ & 9,530 \\
\hline 10298 LOC6498? & 1,079 & $1,32 \mathrm{E}-11$ & $3,30 \mathrm{E}-10$ & 16,236 \\
\hline 16085 SLC45A2 & 1,076 & $1,98 \mathrm{E}-04$ & $6,73 E-04$ & $-0,168$ \\
\hline 19119 ZFP62 & 1,075 & 3,29E-04 & $1,05 \mathrm{E}-03$ & $-0,658$ \\
\hline 7872 ICA1 & 1,075 & $3,78 \mathrm{E}-08$ & $3,35 \mathrm{E}-07$ & 8,281 \\
\hline $11291 \mathrm{MLPH}$ & 1,072 & 3,81E-06 & $1,97 \mathrm{E}-05$ & 3,704 \\
\hline 14174 PTMAP7 & 1,071 & $1,74 \mathrm{E}-12$ & $5,97 \mathrm{E}-11$ & 18,264 \\
\hline 10044 LOC44035 & 1,069 & $3,25 \mathrm{E}-08$ & 2,94E-07 & 8,432 \\
\hline 3892 CORO2A & 1,067 & $2,36 \mathrm{E}-08$ & $2,22 \mathrm{E}-07$ & 8,750 \\
\hline 19564 ZNF692 & 1,067 & $3,14 \mathrm{E}-06$ & $1,66 \mathrm{E}-05$ & 3,895 \\
\hline 9750 LOC2557ع & 1,067 & $2,64 \mathrm{E}-14$ & $1,82 \mathrm{E}-12$ & 22,455 \\
\hline 13087 PDIA5 & 1,067 & $2,02 \mathrm{E}-07$ & $1,45 \mathrm{E}-06$ & 6,615 \\
\hline 3077 CCL3L3 & 1,065 & $1,85 \mathrm{E}-21$ & $1,18 \mathrm{E}-18$ & 38,781 \\
\hline 11953 NDUFB1 & 1,064 & $4,48 \mathrm{E}-14$ & $2,83 \mathrm{E}-12$ & 21,927 \\
\hline 11851 NBPF20 & 1,063 & $8,67 \mathrm{E}-08$ & $6,93 \mathrm{E}-07$ & 7,455 \\
\hline 10043 LOC44034 & 1,062 & $3,13 \mathrm{E}-10$ & $5,18 \mathrm{E}-09$ & 13,069 \\
\hline 7787 HSP90AB & 1,060 & $4,63 \mathrm{E}-14$ & $2,90 \mathrm{E}-12$ & 21,895 \\
\hline 10588 LRRC36 & 1,060 & $1,86 \mathrm{E}-06$ & $1,04 \mathrm{E}-05$ & 4,410 \\
\hline 17166 TDO2 & 1,057 & $6,61 \mathrm{E}-03$ & $1,49 \mathrm{E}-02$ & $-3,510$ \\
\hline 18880 WWC1 & 1,056 & $4,08 \mathrm{E}-05$ & $1,64 \mathrm{E}-04$ & 1,371 \\
\hline 14320 RAB25 & 1,055 & $1,72 \mathrm{E}-12$ & $5,91 \mathrm{E}-11$ & 18,278 \\
\hline 17479 TMEM132 & 1,053 & $8,03 \mathrm{E}-06$ & $3,83 \mathrm{E}-05$ & 2,967 \\
\hline 2788 CAMK2N' & 1,050 & $4,93 \mathrm{E}-11$ & $1,04 \mathrm{E}-09$ & 14,918 \\
\hline 16326 SNRPF & 1,049 & $4,82 \mathrm{E}-13$ & $2,05 \mathrm{E}-11$ & 19,551 \\
\hline 11501 MRPS33 & 1,049 & $1,31 \mathrm{E}-08$ & $1,35 \mathrm{E}-07$ & 9,335 \\
\hline 48 ABCA3 & 1,047 & $9,60 \mathrm{E}-12$ & $2,53 \mathrm{E}-10$ & 16,556 \\
\hline 7781 HSF4 & 1,046 & $6,25 \mathrm{E}-11$ & $1,27 \mathrm{E}-09$ & 14,682 \\
\hline 8262 ISX & 1,046 & $9,49 \mathrm{E}-05$ & $3,48 \mathrm{E}-04$ & 0,547 \\
\hline 16030 SLC35E2 & 1,044 & $1,08 \mathrm{E}-05$ & $4,98 \mathrm{E}-05$ & 2,680 \\
\hline 8799 KLHDC9 & 1,044 & $2,71 \mathrm{E}-11$ & $6,14 \mathrm{E}-10$ & 15,517 \\
\hline 14015 PRR7 & 1,043 & $2,46 \mathrm{E}-11$ & $5,62 \mathrm{E}-10$ & 15,616 \\
\hline 11264 MKNK2 & 1,040 & $1,28 \mathrm{E}-08$ & $1,32 \mathrm{E}-07$ & 9,360 \\
\hline 14947 RP11-216I & 1,039 & $4,48 \mathrm{E}-09$ & $5,27 \mathrm{E}-08$ & 10,409 \\
\hline 10098 LOC4424t & 1,037 & $1,66 \mathrm{E}-07$ & $1,22 \mathrm{E}-06$ & 6,811 \\
\hline 6568 GAS5 & 1,036 & $1,72 \mathrm{E}-06$ & $9,74 \mathrm{E}-06$ & 4,487 \\
\hline
\end{tabular}




\begin{tabular}{|c|c|c|c|c|}
\hline 7572 HLA-DMF & 1,033 & $8,46 \mathrm{E}-07$ & $5,15 \mathrm{E}-06$ & 5,192 \\
\hline 1344 BCAM & 1,030 & $1,75 \mathrm{E}-11$ & $4,23 \mathrm{E}-10$ & 15,957 \\
\hline 1726 C12orf45 & 1,029 & $1,80 \mathrm{E}-11$ & $4,32 \mathrm{E}-10$ & 15,926 \\
\hline 10002 LOC40135 & 1,029 & $2,24 \mathrm{E}-13$ & $1,06 \mathrm{E}-11$ & 20,318 \\
\hline 1517 BRD9 & 1,028 & $3,07 \mathrm{E}-10$ & $5,10 \mathrm{E}-09$ & 13,088 \\
\hline 7540 HIST1H4F & 1,027 & $1,46 \mathrm{E}-04$ & $5,13 \mathrm{E}-04$ & 0,125 \\
\hline 18051 TSPAN13 & 1,026 & $1,58 \mathrm{E}-06$ & $9,03 \mathrm{E}-06$ & 4,572 \\
\hline 9183 LIG1 & 1,026 & $5,63 \mathrm{E}-11$ & $1,17 \mathrm{E}-09$ & 14,786 \\
\hline 8319 ITPR3 & 1,025 & $1,17 \mathrm{E}-09$ & $1,65 \mathrm{E}-08$ & 11,748 \\
\hline $12224 \mathrm{NP}$ & 1,024 & $1,92 \mathrm{E}-05$ & $8,34 \mathrm{E}-05$ & 2,112 \\
\hline 5376 ERCC6L & 1,023 & $4,60 \mathrm{E}-09$ & $5,39 \mathrm{E}-08$ & 10,383 \\
\hline 17109 TCEA3 & 1,022 & $1,57 \mathrm{E}-07$ & $1,17 \mathrm{E}-06$ & 6,863 \\
\hline 452 AK3L1 & 1,022 & $5,62 \mathrm{E}-08$ & $4,76 \mathrm{E}-07$ & 7,886 \\
\hline 697 ANKZF1 & 1,021 & $9,27 \mathrm{E}-11$ & $1,81 \mathrm{E}-09$ & 14,287 \\
\hline 2221 C20orf74 & 1,021 & $8,03 \mathrm{E}-05$ & $3,00 \mathrm{E}-04$ & 0,710 \\
\hline 9902 LOC3885t & 1,019 & $3,15 \mathrm{E}-10$ & $5,21 \mathrm{E}-09$ & 13,062 \\
\hline 1392 BDH1 & 1,018 & $5,67 \mathrm{E}-12$ & $1,63 \mathrm{E}-10$ & 17,084 \\
\hline 7575 HLA-DPA & 1,017 & $8,65 \mathrm{E}-08$ & $6,92 \mathrm{E}-07$ & 7,457 \\
\hline 11064 MELK & 1,017 & $8,26 \mathrm{E}-08$ & $6,65 \mathrm{E}-07$ & 7,503 \\
\hline 8251 ISG15 & 1,016 & $1,38 \mathrm{E}-04$ & $4,88 \mathrm{E}-04$ & 0,181 \\
\hline 11928 NDRG1 & 1,016 & $5,78 \mathrm{E}-04$ & $1,73 \mathrm{E}-03$ & $-1,202$ \\
\hline 14953 RP11-345I & 1,015 & $2,41 \mathrm{E}-06$ & $1,31 \mathrm{E}-05$ & 4,154 \\
\hline 13551 PMS2L2 & 1,013 & $5,34 \mathrm{E}-17$ & $9,49 \mathrm{E}-15$ & 28,641 \\
\hline 7183 GTF2H2D & 1,013 & 4,92E-09 & $5,72 \mathrm{E}-08$ & 10,316 \\
\hline 15950 SLC25A3 & 1,010 & $1,18 \mathrm{E}-13$ & $6,29 \mathrm{E}-12$ & 20,954 \\
\hline 8095 IL4I1 & 1,010 & $6,71 \mathrm{E}-08$ & $5,58 \mathrm{E}-07$ & 7,709 \\
\hline 12346 NSUN5C & 1,010 & $6,07 \mathrm{E}-17$ & $1,05 \mathrm{E}-14$ & 28,513 \\
\hline 5504 FABP1 & 1,009 & $1,34 \mathrm{E}-02$ & $2,75 \mathrm{E}-02$ & $-4,160$ \\
\hline 11271 MLEC & 1,008 & $5,84 \mathrm{E}-09$ & $6,69 \mathrm{E}-08$ & 10,144 \\
\hline 18048 TSPAN10 & 1,008 & $6,65 \mathrm{E}-14$ & $3,99 \mathrm{E}-12$ & 21,531 \\
\hline 15457 SEC61G & 1,008 & 4,39E-07 & 2,91E-06 & 5,843 \\
\hline 3915 COX6C & 1,006 & $2,19 \mathrm{E}-11$ & $5,15 \mathrm{E}-10$ & 15,731 \\
\hline 902 ARHGEF1 & 1,004 & $9,57 \mathrm{E}-10$ & $1,38 \mathrm{E}-08$ & 11,952 \\
\hline 12167 NMRAL1 & 1,004 & $1,65 \mathrm{E}-11$ & $4,03 \mathrm{E}-10$ & 16,012 \\
\hline 10099 LOC44257 & 1,003 & $1,30 \mathrm{E}-11$ & $3,26 \mathrm{E}-10$ & 16,254 \\
\hline 2854 CASP10 & 1,003 & $1,58 \mathrm{E}-04$ & $5,50 \mathrm{E}-04$ & 0,050 \\
\hline 9787 LOC28423 & 1,003 & $1,21 \mathrm{E}-11$ & $3,08 \mathrm{E}-10$ & 16,324 \\
\hline 177 ACP5 & $-1,002$ & $1,98 \mathrm{E}-04$ & $6,71 \mathrm{E}-04$ & $-0,166$ \\
\hline 19536 ZNF655 & $-1,003$ & $2,68 \mathrm{E}-09$ & $3,37 \mathrm{E}-08$ & 10,922 \\
\hline 16717 STEAP3 & $-1,003$ & $3,11 \mathrm{E}-09$ & $3,84 \mathrm{E}-08$ & 10,774 \\
\hline 621 ANGPTL7 & $-1,003$ & $2,55 \mathrm{E}-09$ & $3,24 \mathrm{E}-08$ & 10,973 \\
\hline 13810 PPP3CA & $-1,003$ & $2,96 \mathrm{E}-05$ & $1,23 \mathrm{E}-04$ & 1,685 \\
\hline 4857 DOK7 & $-1,004$ & $5,21 \mathrm{E}-14$ & $3,23 \mathrm{E}-12$ & 21,775 \\
\hline
\end{tabular}




\begin{tabular}{|c|c|c|c|c|}
\hline 16182 SLIT2 & $-1,004$ & $1,48 \mathrm{E}-11$ & $3,65 \mathrm{E}-10$ & 16,125 \\
\hline $17221 \mathrm{TF}$ & $-1,005$ & $1,41 \mathrm{E}-06$ & $8,16 \mathrm{E}-06$ & 4,685 \\
\hline 15897 SLC22A1 & $-1,007$ & $1,40 \mathrm{E}-05$ & $6,30 \mathrm{E}-05$ & 2,418 \\
\hline 15865 SLC16A9 & $-1,008$ & $2,80 \mathrm{E}-06$ & $1,49 \mathrm{E}-05$ & 4,007 \\
\hline 12293 NR2F2 & $-1,008$ & 2,99E-08 & $2,73 \mathrm{E}-07$ & 8,516 \\
\hline 16618 SSBP2 & $-1,008$ & $4,57 \mathrm{E}-10$ & 7,21E-09 & 12,691 \\
\hline 16777 STX12 & $-1,009$ & $4,01 \mathrm{E}-14$ & $2,59 \mathrm{E}-12$ & 22,038 \\
\hline 7181 GTF2F2 & $-1,010$ & $1,20 \mathrm{E}-10$ & $2,26 \mathrm{E}-09$ & 14,028 \\
\hline 17840 TPST2 & $-1,010$ & $3,04 \mathrm{E}-15$ & $2,90 \mathrm{E}-13$ & 24,614 \\
\hline 8333 JAGN1 & $-1,011$ & $1,18 \mathrm{E}-09$ & $1,66 \mathrm{E}-08$ & 11,742 \\
\hline 17254 TGFB2 & $-1,011$ & $2,86 \mathrm{E}-10$ & 4,83E-09 & 13,161 \\
\hline 14716 RGS5 & $-1,011$ & $5,91 \mathrm{E}-06$ & $2,91 \mathrm{E}-05$ & 3,270 \\
\hline 3848 COLEC12 & $-1,011$ & $1,10 \mathrm{E}-02$ & 2,32E-02 & $-3,978$ \\
\hline 17259 TGFBR3 & $-1,012$ & $5,29 \mathrm{E}-10$ & $8,23 E-09$ & 12,545 \\
\hline 17832 TPRG1 & $-1,012$ & $3,29 \mathrm{E}-12$ & $1,02 \mathrm{E}-10$ & 17,628 \\
\hline 4681 DKFZP581 & $-1,013$ & $3,92 \mathrm{E}-10$ & $6,29 \mathrm{E}-09$ & 12,844 \\
\hline 19113 ZFP36L1 & $-1,013$ & $2,22 \mathrm{E}-10$ & $3,85 \mathrm{E}-09$ & 13,413 \\
\hline 17652 TMOD1 & $-1,014$ & $3,23 \mathrm{E}-11$ & $7,17 \mathrm{E}-10$ & 15,341 \\
\hline 8384 KANK2 & $-1,015$ & $3,45 \mathrm{E}-14$ & $2,31 \mathrm{E}-12$ & 22,186 \\
\hline 9150 LGALS7B & $-1,016$ & $1,43 \mathrm{E}-08$ & $1,45 \mathrm{E}-07$ & 9,251 \\
\hline 17290 THBS4 & $-1,017$ & $4,57 \mathrm{E}-03$ & $1,08 \mathrm{E}-02$ & $-3,166$ \\
\hline 5285 ENPP1 & $-1,017$ & $4,13 \mathrm{E}-08$ & $3,63 \mathrm{E}-07$ & 8,193 \\
\hline 6338 FOXN3 & $-1,017$ & $1,04 \mathrm{E}-07$ & $8,15 \mathrm{E}-07$ & 7,273 \\
\hline 3319 CDKL1 & $-1,018$ & $1,21 \mathrm{E}-11$ & $3,07 \mathrm{E}-10$ & 16,329 \\
\hline 5245 EMCN & $-1,018$ & $1,16 \mathrm{E}-06$ & $6,85 \mathrm{E}-06$ & 4,880 \\
\hline 7088 GRHL1 & $-1,020$ & $1,49 \mathrm{E}-06$ & $8,54 \mathrm{E}-06$ & 4,634 \\
\hline 320 ADH1A & $-1,021$ & $3,88 \mathrm{E}-06$ & $2,00 \mathrm{E}-05$ & 3,685 \\
\hline 16669 ST8SIA1 & $-1,021$ & $3,83 \mathrm{E}-05$ & $1,55 \mathrm{E}-04$ & 1,433 \\
\hline 6374 FRMD6 & $-1,022$ & $1,86 \mathrm{E}-17$ & $4,03 \mathrm{E}-15$ & 29,690 \\
\hline 111 ABLIM1 & $-1,022$ & $2,66 \mathrm{E}-06$ & $1,42 \mathrm{E}-05$ & 4,059 \\
\hline 14025 PRRX2 & $-1,022$ & $4,93 \mathrm{E}-10$ & 7,73E-09 & 12,615 \\
\hline 3604 CKMT2 & $-1,022$ & $3,44 \mathrm{E}-05$ & $1,41 \mathrm{E}-04$ & 1,538 \\
\hline 16287 SNCAIP & $-1,023$ & $7,21 \mathrm{E}-11$ & $1,44 \mathrm{E}-09$ & 14,539 \\
\hline 13703 PPAP2A & $-1,024$ & $1,24 \mathrm{E}-02$ & 2,57E-02 & $-4,087$ \\
\hline 8793 KLHDC5 & $-1,025$ & $9,28 \mathrm{E}-14$ & $5,20 \mathrm{E}-12$ & 21,199 \\
\hline $18595 \mathrm{VCL}$ & $-1,027$ & $1,35 \mathrm{E}-10$ & 2,51E-09 & 13,908 \\
\hline 1266 B3GNT8 & $-1,027$ & $9,64 \mathrm{E}-17$ & $1,56 \mathrm{E}-14$ & 28,053 \\
\hline 3613 CLCA4 & $-1,027$ & $4,60 \mathrm{E}-06$ & $2,34 \mathrm{E}-05$ & 3,516 \\
\hline 12043 NFIA & $-1,027$ & $4,77 \mathrm{E}-08$ & $4,12 \mathrm{E}-07$ & 8,050 \\
\hline 14032 PRSS23 & $-1,027$ & $1,11 \mathrm{E}-04$ & $4,02 \mathrm{E}-04$ & 0,391 \\
\hline 8279 ITGA8 & $-1,028$ & $6,25 \mathrm{E}-08$ & $5,23 \mathrm{E}-07$ & 7,781 \\
\hline 16898 SYT10 & $-1,031$ & $1,03 \mathrm{E}-08$ & $1,09 \mathrm{E}-07$ & 9,575 \\
\hline 10442 LOC72997 & $-1,033$ & $1,33 \mathrm{E}-10$ & $2,48 \mathrm{E}-09$ & 13,922 \\
\hline
\end{tabular}




\begin{tabular}{|c|c|c|c|c|}
\hline 7886 ID3 & $-1,033$ & $6,97 \mathrm{E}-08$ & $5,75 \mathrm{E}-07$ & 7,672 \\
\hline 4165 CTPS & $-1,035$ & $2,28 \mathrm{E}-07$ & $1,63 \mathrm{E}-06$ & 6,491 \\
\hline 15539 SERP2 & $-1,036$ & $6,82 \mathrm{E}-05$ & 2,59E-04 & 0,869 \\
\hline 9249 LMNA & $-1,037$ & $1,75 \mathrm{E}-12$ & $5,98 \mathrm{E}-11$ & 18,260 \\
\hline 2733 CACNA2I & $-1,038$ & $3,47 \mathrm{E}-09$ & $4,21 \mathrm{E}-08$ & 10,666 \\
\hline 15095 RPS27L & $-1,039$ & 2,94E-09 & $3,66 \mathrm{E}-08$ & 10,831 \\
\hline 18143 TTN & $-1,040$ & $1,91 \mathrm{E}-04$ & $6,51 \mathrm{E}-04$ & $-0,133$ \\
\hline 14110 PSMD7 & $-1,041$ & $1,61 \mathrm{E}-08$ & $1,61 \mathrm{E}-07$ & 9,134 \\
\hline 1238 AXL & $-1,041$ & $2,60 \mathrm{E}-09$ & $3,29 \mathrm{E}-08$ & 10,954 \\
\hline 7906 IFFO2 & $-1,042$ & $1,54 \mathrm{E}-07$ & $1,15 \mathrm{E}-06$ & 6,882 \\
\hline 17724 TNIK & $-1,043$ & $2,35 \mathrm{E}-15$ & $2,37 \mathrm{E}-13$ & 24,870 \\
\hline 8239 IRS1 & $-1,043$ & $1,62 \mathrm{E}-06$ & $9,21 \mathrm{E}-06$ & 4,550 \\
\hline 15699 SH3BGRL & $-1,044$ & $5,91 \mathrm{E}-11$ & $1,22 \mathrm{E}-09$ & 14,737 \\
\hline 1850 C15orf41 & $-1,045$ & $1,36 \mathrm{E}-12$ & $4,88 \mathrm{E}-11$ & 18,511 \\
\hline 9255 LMO4 & $-1,045$ & $1,82 \mathrm{E}-09$ & $2,44 \mathrm{E}-08$ & 11,308 \\
\hline 53 ABCA8 & $-1,046$ & $3,22 \mathrm{E}-09$ & $3,96 \mathrm{E}-08$ & 10,739 \\
\hline 7164 GSTO1 & $-1,048$ & $1,93 \mathrm{E}-09$ & $2,55 \mathrm{E}-08$ & 11,253 \\
\hline 18622 VILL & $-1,048$ & $3,89 \mathrm{E}-10$ & $6,25 \mathrm{E}-09$ & 12,853 \\
\hline 5074 EDNRA & $-1,049$ & $1,14 \mathrm{E}-12$ & $4,21 \mathrm{E}-11$ & 18,690 \\
\hline 18963 YPEL5 & $-1,049$ & $8,65 \mathrm{E}-11$ & $1,70 \mathrm{E}-09$ & 14,357 \\
\hline 116 ABR & $-1,050$ & 1,99E-09 & $2,62 \mathrm{E}-08$ & 11,220 \\
\hline 7453 HGD & $-1,052$ & $6,74 \mathrm{E}-04$ & $1,98 \mathrm{E}-03$ & $-1,349$ \\
\hline 13048 PDE11A & $-1,054$ & $4,31 \mathrm{E}-06$ & $2,21 \mathrm{E}-05$ & 3,580 \\
\hline 12552 OPTN & $-1,055$ & $6,81 \mathrm{E}-07$ & $4,28 \mathrm{E}-06$ & 5,407 \\
\hline 12121 NKX3-1 & $-1,057$ & $6,23 \mathrm{E}-06$ & $3,05 \mathrm{E}-05$ & 3,217 \\
\hline 1416 BEX4 & $-1,057$ & $7,04 \mathrm{E}-08$ & $5,81 \mathrm{E}-07$ & 7,662 \\
\hline 5286 ENPP2 & $-1,057$ & $3,74 \mathrm{E}-04$ & $1,18 \mathrm{E}-03$ & $-0,784$ \\
\hline 13332 PIK3R1 & $-1,058$ & $1,12 \mathrm{E}-11$ & $2,87 \mathrm{E}-10$ & 16,406 \\
\hline 6071 FLJ10357 & $-1,059$ & $3,41 \mathrm{E}-09$ & $4,17 \mathrm{E}-08$ & 10,681 \\
\hline 12828 PAK3 & $-1,060$ & $1,12 \mathrm{E}-08$ & $1,17 \mathrm{E}-07$ & 9,498 \\
\hline 14458 RARRES2 & $-1,062$ & 3,01E-09 & $3,74 \mathrm{E}-08$ & 10,807 \\
\hline 10962 MCAM & $-1,063$ & $6,78 \mathrm{E}-14$ & $4,03 \mathrm{E}-12$ & 21,512 \\
\hline 13443 PLCE1 & $-1,064$ & $1,24 \mathrm{E}-09$ & $1,72 \mathrm{E}-08$ & 11,696 \\
\hline 2867 CASQ2 & $-1,065$ & $5,00 \mathrm{E}-08$ & 4,29E-07 & 8,003 \\
\hline 18212 TWIST2 & $-1,067$ & $5,24 \mathrm{E}-11$ & $1,10 \mathrm{E}-09$ & 14,858 \\
\hline 18734 WDR1 & $-1,069$ & $2,81 \mathrm{E}-16$ & $3,85 \mathrm{E}-14$ & 26,989 \\
\hline 5115 EFS & $-1,069$ & $1,28 \mathrm{E}-07$ & 9,77E-07 & 7,066 \\
\hline 215 ACTN1 & $-1,070$ & $8,66 \mathrm{E}-10$ & $1,26 \mathrm{E}-08$ & 12,052 \\
\hline 476 AKR1C1 & $-1,070$ & $1,58 \mathrm{E}-09$ & $2,15 \mathrm{E}-08$ & 11,449 \\
\hline 13866 PRDM6 & $-1,070$ & $3,25 \mathrm{E}-10$ & $5,34 \mathrm{E}-09$ & 13,033 \\
\hline 18239 TXNRD1 & $-1,071$ & $2,57 \mathrm{E}-08$ & 2,39E-07 & 8,665 \\
\hline 17755 TNS1 & $-1,072$ & $1,66 \mathrm{E}-11$ & $4,05 \mathrm{E}-10$ & 16,007 \\
\hline 14773 RILPL2 & $-1,073$ & $1,18 \mathrm{E}-10$ & 2,24E-09 & 14,045 \\
\hline
\end{tabular}




\begin{tabular}{|c|c|c|c|c|}
\hline 14971 RP4-691N & $-1,075$ & $1,47 \mathrm{E}-05$ & $6,56 \mathrm{E}-05$ & 2,375 \\
\hline $12215 \mathrm{NOV}$ & $-1,075$ & 3,33E-06 & $1,75 \mathrm{E}-05$ & 3,835 \\
\hline 704 ANO5 & $-1,077$ & $2,16 \mathrm{E}-14$ & $1,54 \mathrm{E}-12$ & 22,655 \\
\hline $55 \mathrm{ABCB} 1$ & $-1,077$ & $3,05 \mathrm{E}-18$ & $8,85 \mathrm{E}-16$ & 31,482 \\
\hline 5844 FBN1 & $-1,078$ & $6,67 \mathrm{E}-05$ & $2,54 \mathrm{E}-04$ & 0,891 \\
\hline 7408 HECTD2 & $-1,078$ & $2,35 \mathrm{E}-13$ & $1,10 \mathrm{E}-11$ & 20,267 \\
\hline 5632 FAM162B & $-1,079$ & $1,57 \mathrm{E}-10$ & $2,85 \mathrm{E}-09$ & 13,759 \\
\hline 16379 SOD3 & $-1,080$ & $5,13 \mathrm{E}-07$ & 3,33E-06 & 5,688 \\
\hline 172 ACOX2 & $-1,083$ & $2,07 \mathrm{E}-08$ & 1,99E-07 & 8,884 \\
\hline 5039 EBF4 & $-1,085$ & $1,79 \mathrm{E}-12$ & $6,10 \mathrm{E}-11$ & 18,236 \\
\hline 13811 РРР3СВ & $-1,087$ & $2,86 \mathrm{E}-12$ & $9,00 \mathrm{E}-11$ & 17,769 \\
\hline 9166 LHFP & $-1,088$ & $5,69 \mathrm{E}-07$ & $3,66 \mathrm{E}-06$ & 5,585 \\
\hline 17581 TMEM35 & $-1,091$ & $4,12 \mathrm{E}-12$ & $1,22 \mathrm{E}-10$ & 17,404 \\
\hline 7066 GPX3 & $-1,092$ & $6,47 \mathrm{E}-09$ & 7,32E-08 & 10,043 \\
\hline 8101 IL6ST & $-1,093$ & $1,87 \mathrm{E}-12$ & $6,31 \mathrm{E}-11$ & 18,195 \\
\hline 7940 IFNGR1 & $-1,093$ & $1,78 \mathrm{E}-07$ & $1,30 \mathrm{E}-06$ & 6,737 \\
\hline 3352 CEACAM & $-1,094$ & $5,06 \mathrm{E}-08$ & 4,34E-07 & 7,991 \\
\hline 12303 NRAP & $-1,096$ & $6,77 \mathrm{E}-04$ & 1,99E-03 & $-1,354$ \\
\hline 1325 BATF & $-1,098$ & 9,70E-06 & $4,53 \mathrm{E}-05$ & 2,782 \\
\hline 18681 VSIG2 & $-1,098$ & $3,45 \mathrm{E}-08$ & 3,09E-07 & 8,372 \\
\hline 18353 UCHL1 & $-1,100$ & $2,08 \mathrm{E}-04$ & 7,01E-04 & $-0,214$ \\
\hline 13442 PLCD4 & $-1,101$ & $2,47 \mathrm{E}-10$ & $4,25 \mathrm{E}-09$ & 13,305 \\
\hline 13389 PKIG & $-1,102$ & $2,25 \mathrm{E}-10$ & 3,91E-09 & 13,398 \\
\hline 5075 EDNRB & $-1,102$ & $4,40 \mathrm{E}-09$ & $5,18 \mathrm{E}-08$ & 10,428 \\
\hline 12365 NTN1 & $-1,102$ & $1,80 \mathrm{E}-16$ & $2,65 \mathrm{E}-14$ & 27,431 \\
\hline 14163 PTGS2 & $-1,103$ & $1,24 \mathrm{E}-05$ & $5,64 \mathrm{E}-05$ & 2,542 \\
\hline 4976 DUSP23 & $-1,104$ & $1,56 \mathrm{E}-04$ & $5,45 \mathrm{E}-04$ & 0,061 \\
\hline 17809 TPBG & $-1,104$ & $2,17 \mathrm{E}-10$ & 3,78E-09 & 13,435 \\
\hline $180 \mathrm{ACPP}$ & $-1,105$ & $5,14 \mathrm{E}-03$ & $1,19 \mathrm{E}-02$ & $-3,276$ \\
\hline 10698 LY6K & $-1,105$ & $1,15 \mathrm{E}-02$ & $2,42 \mathrm{E}-02$ & $-4,024$ \\
\hline 15916 SLC24A3 & $-1,105$ & $6,32 \mathrm{E}-18$ & $1,66 \mathrm{E}-15$ & 30,761 \\
\hline 639 ANKRD1 & $-1,109$ & $1,53 \mathrm{E}-03$ & $4,12 \mathrm{E}-03$ & $-2,134$ \\
\hline 112 ABLIM2 & $-1,109$ & $1,06 \mathrm{E}-05$ & $4,92 \mathrm{E}-05$ & 2,693 \\
\hline 18863 WNT5B & $-1,110$ & $4,29 \mathrm{E}-11$ & $9,19 \mathrm{E}-10$ & 15,058 \\
\hline 2088 C1orf190 & $-1,110$ & $3,69 \mathrm{E}-15$ & $3,38 \mathrm{E}-13$ & 24,421 \\
\hline 16605 SRPX2 & $-1,110$ & 3,22E-09 & $3,96 \mathrm{E}-08$ & 10,738 \\
\hline 7907 IFI16 & $-1,111$ & $3,12 \mathrm{E}-08$ & 2,84E-07 & 8,473 \\
\hline 14858 RNF150 & $-1,111$ & $7,98 \mathrm{E}-10$ & $1,17 \mathrm{E}-08$ & 12,133 \\
\hline $3281 \mathrm{CDH} 13$ & $-1,112$ & $1,57 \mathrm{E}-07$ & $1,17 \mathrm{E}-06$ & 6,863 \\
\hline 17399 TM4SF1 & $-1,112$ & $5,67 \mathrm{E}-06$ & $2,81 \mathrm{E}-05$ & 3,310 \\
\hline 1430 ВHMT2 & $-1,116$ & $4,06 \mathrm{E}-17$ & $7,69 \mathrm{E}-15$ & 28,914 \\
\hline 2870 CAST & $-1,119$ & $5,88 \mathrm{E}-10$ & $9,02 \mathrm{E}-09$ & 12,440 \\
\hline 15619 SFRP4 & $-1,122$ & $6,35 \mathrm{E}-05$ & $2,43 \mathrm{E}-04$ & 0,940 \\
\hline
\end{tabular}




\begin{tabular}{|c|c|c|c|c|}
\hline 907 ARHGEF & $-1,124$ & $1,76 \mathrm{E}-10$ & $3,15 \mathrm{E}-09$ & 13,646 \\
\hline 12367 NTN4 & $-1,127$ & $2,35 \mathrm{E}-07$ & $1,67 \mathrm{E}-06$ & 6,464 \\
\hline 17591 TMEM43 & $-1,127$ & $5,65 \mathrm{E}-15$ & $4,79 \mathrm{E}-13$ & 23,995 \\
\hline 852 AREG & $-1,127$ & $3,80 \mathrm{E}-04$ & 1,19E-03 & $-0,798$ \\
\hline 15305 SAV1 & $-1,128$ & $4,19 \mathrm{E}-10$ & 6,67E-09 & 12,778 \\
\hline 8783 KLF5 & $-1,128$ & $7,67 \mathrm{E}-12$ & $2,10 \mathrm{E}-10$ & 16,781 \\
\hline 716 ANTXR2 & $-1,133$ & $3,71 \mathrm{E}-14$ & $2,46 \mathrm{E}-12$ & 22,114 \\
\hline 9023 LAMC1 & $-1,134$ & $2,45 \mathrm{E}-10$ & 4,21E-09 & 13,316 \\
\hline 4732 DMD & $-1,134$ & $1,60 \mathrm{E}-13$ & $7,95 \mathrm{E}-12$ & 20,656 \\
\hline 14424 RAMP1 & $-1,134$ & $1,01 \mathrm{E}-03$ & $2,85 \mathrm{E}-03$ & $-1,736$ \\
\hline 16046 SLC38A1 & $-1,135$ & $1,24 \mathrm{E}-06$ & 7,26E-06 & 4,812 \\
\hline 1261 B3GNT3 & $-1,135$ & $3,92 \mathrm{E}-06$ & $2,02 \mathrm{E}-05$ & 3,674 \\
\hline 17499 TMEM14( & $-1,136$ & $5,57 \mathrm{E}-06$ & $2,77 \mathrm{E}-05$ & 3,327 \\
\hline 5507 FABP3 & $-1,136$ & $9,09 \mathrm{E}-16$ & $1,04 \mathrm{E}-13$ & 25,818 \\
\hline 16364 SNX7 & $-1,136$ & $1,18 \mathrm{E}-07$ & $9,12 \mathrm{E}-07$ & 7,145 \\
\hline 13448 PLCL1 & $-1,137$ & 3,87E-09 & $4,62 \mathrm{E}-08$ & 10,557 \\
\hline 8331 JAG1 & $-1,138$ & $7,46 \mathrm{E}-08$ & $6,10 \mathrm{E}-07$ & 7,604 \\
\hline 505 ALDH3A2 & $-1,139$ & $5,95 \mathrm{E}-11$ & $1,23 \mathrm{E}-09$ & 14,731 \\
\hline $2711 \mathrm{CAB} 39 \mathrm{~L}$ & $-1,139$ & $1,65 \mathrm{E}-09$ & 2,23E-08 & 11,408 \\
\hline 9036 LARGE & $-1,139$ & $4,24 \mathrm{E}-11$ & $9,11 \mathrm{E}-10$ & 15,071 \\
\hline 12478 OCC-1 & $-1,140$ & $2,56 \mathrm{E}-09$ & $3,24 \mathrm{E}-08$ & 10,970 \\
\hline 1480 BNC2 & $-1,140$ & $1,35 \mathrm{E}-17$ & $3,05 \mathrm{E}-15$ & 30,010 \\
\hline 11063 MEIS3P1 & $-1,145$ & $7,60 \mathrm{E}-12$ & $2,09 \mathrm{E}-10$ & 16,790 \\
\hline 3432 CFD & $-1,146$ & $5,85 \mathrm{E}-04$ & $1,74 \mathrm{E}-03$ & $-1,214$ \\
\hline 2275 C22orf28 & $-1,147$ & $3,11 \mathrm{E}-15$ & $2,94 \mathrm{E}-13$ & 24,590 \\
\hline 8096 IL4R & $-1,147$ & $6,41 \mathrm{E}-04$ & $1,90 \mathrm{E}-03$ & $-1,302$ \\
\hline 11324 MMP3 & $-1,147$ & $1,42 \mathrm{E}-05$ & $6,38 \mathrm{E}-05$ & 2,405 \\
\hline 1289 BAG1 & $-1,147$ & $6,51 \mathrm{E}-11$ & 1,32E-09 & 14,640 \\
\hline 19240 ZNF219 & $-1,149$ & $3,94 \mathrm{E}-14$ & $2,56 \mathrm{E}-12$ & 22,055 \\
\hline 6999 GPR177 & $-1,150$ & $6,84 \mathrm{E}-10$ & $1,03 \mathrm{E}-08$ & 12,288 \\
\hline 1000 ART3 & $-1,155$ & $1,60 \mathrm{E}-10$ & 2,88E-09 & 13,743 \\
\hline 15160 RSPO1 & $-1,155$ & $1,56 \mathrm{E}-13$ & $7,78 \mathrm{E}-12$ & 20,682 \\
\hline 823 APP & $-1,159$ & 4,77E-06 & 2,41E-05 & 3,482 \\
\hline 1366 BCL2 & $-1,159$ & $5,95 \mathrm{E}-12$ & $1,70 \mathrm{E}-10$ & 17,035 \\
\hline 7152 GSTA1 & $-1,160$ & $1,18 \mathrm{E}-08$ & $1,23 \mathrm{E}-07$ & 9,443 \\
\hline 7975 IGFBP7 & $-1,160$ & $1,04 \mathrm{E}-06$ & $6,20 \mathrm{E}-06$ & 4,986 \\
\hline 12917 PBX1 & $-1,161$ & $4,28 \mathrm{E}-12$ & $1,26 \mathrm{E}-10$ & 17,366 \\
\hline 12050 NFKBIA & $-1,162$ & $3,28 \mathrm{E}-08$ & $2,96 \mathrm{E}-07$ & 8,423 \\
\hline 4219 CXCL1 & $-1,162$ & $5,34 \mathrm{E}-03$ & $1,23 \mathrm{E}-02$ & $-3,311$ \\
\hline 17738 TNNI2 & $-1,163$ & 2,77E-04 & $9,03 \mathrm{E}-04$ & $-0,493$ \\
\hline 16202 SMAD3 & $-1,163$ & $4,80 \mathrm{E}-17$ & $8,67 \mathrm{E}-15$ & 28,748 \\
\hline 6250 FLJ46111 & $-1,168$ & $7,19 \mathrm{E}-14$ & $4,21 \mathrm{E}-12$ & 21,453 \\
\hline 5998 FGFBP1 & $-1,168$ & $1,70 \mathrm{E}-12$ & $5,88 \mathrm{E}-11$ & 18,288 \\
\hline
\end{tabular}




\begin{tabular}{|c|c|c|c|c|}
\hline 19143 ZHX3 & $-1,170$ & $1,49 \mathrm{E}-12$ & $5,26 \mathrm{E}-11$ & 18,420 \\
\hline 11854 NBR1 & $-1,170$ & $1,90 \mathrm{E}-12$ & $6,38 \mathrm{E}-11$ & 18,176 \\
\hline 4405 DBNDD2 & $-1,170$ & $4,16 \mathrm{E}-15$ & $3,68 \mathrm{E}-13$ & 24,302 \\
\hline 500 ALDH1B1 & $-1,172$ & $3,59 \mathrm{E}-12$ & $1,09 \mathrm{E}-10$ & 17,540 \\
\hline 15445 SEC23A & $-1,173$ & $1,02 \mathrm{E}-11$ & $2,68 \mathrm{E}-10$ & 16,494 \\
\hline 15706 SH3D19 & $-1,175$ & $2,31 \mathrm{E}-12$ & $7,51 \mathrm{E}-11$ & 17,983 \\
\hline 9890 LOC38776 & $-1,175$ & $8,22 \mathrm{E}-04$ & 2,37E-03 & $-1,539$ \\
\hline 1602 C10orf116 & $-1,176$ & 2,37E-04 & $7,85 \mathrm{E}-04$ & $-0,340$ \\
\hline 773 APCDD1 & $-1,176$ & $2,45 \mathrm{E}-07$ & $1,73 \mathrm{E}-06$ & 6,423 \\
\hline 8718 KIF1C & $-1,177$ & $2,08 \mathrm{E}-14$ & $1,49 \mathrm{E}-12$ & 22,692 \\
\hline 18235 TXNIP & $-1,177$ & $1,73 \mathrm{E}-05$ & $7,63 \mathrm{E}-05$ & 2,210 \\
\hline 12331 NSBP1 & $-1,181$ & $7,15 \mathrm{E}-16$ & $8,59 \mathrm{E}-14$ & 26,058 \\
\hline 6857 GNG4 & $-1,183$ & $1,42 \mathrm{E}-05$ & $6,39 \mathrm{E}-05$ & 2,404 \\
\hline 18224 TXNDC13 & $-1,185$ & $2,54 \mathrm{E}-15$ & $2,49 \mathrm{E}-13$ & 24,794 \\
\hline 7324 hCG_1783 & $-1,186$ & $1,88 \mathrm{E}-13$ & $9,06 \mathrm{E}-12$ & 20,495 \\
\hline 7970 IGFBP2 & $-1,186$ & $2,55 \mathrm{E}-08$ & 2,37E-07 & 8,675 \\
\hline 14982 RP9P & $-1,188$ & $3,86 \mathrm{E}-22$ & $3,63 \mathrm{E}-19$ & 40,314 \\
\hline 9019 LAMB2 & $-1,188$ & $3,98 \mathrm{E}-12$ & $1,19 \mathrm{E}-10$ & 17,437 \\
\hline 5792 FAM92A1 & $-1,188$ & $1,90 \mathrm{E}-12$ & $6,37 \mathrm{E}-11$ & 18,180 \\
\hline 12329 NRXN3 & $-1,190$ & 7,51E-04 & 2,19E-03 & $-1,454$ \\
\hline 6474 GABARA & $-1,191$ & $8,47 \mathrm{E}-09$ & $9,26 \mathrm{E}-08$ & 9,774 \\
\hline 6524 GALNAC & $-1,192$ & $2,95 \mathrm{E}-11$ & $6,61 \mathrm{E}-10$ & 15,434 \\
\hline 17352 TIMP3 & $-1,192$ & 3,83E-09 & $4,59 \mathrm{E}-08$ & 10,566 \\
\hline 7361 HCLS1 & $-1,195$ & $1,50 \mathrm{E}-05$ & $6,69 \mathrm{E}-05$ & 2,354 \\
\hline 615 ANGPTL1 & $-1,195$ & $1,03 \mathrm{E}-10$ & $1,98 \mathrm{E}-09$ & 14,183 \\
\hline 10505 LOXL1 & $-1,196$ & $3,35 \mathrm{E}-08$ & 3,01E-07 & 8,403 \\
\hline 6729 GJA1 & $-1,198$ & $2,02 \mathrm{E}-12$ & $6,75 \mathrm{E}-11$ & 18,115 \\
\hline 15237 S100B & $-1,198$ & $8,03 \mathrm{E}-12$ & $2,18 \mathrm{E}-10$ & 16,735 \\
\hline 14624 RELB & $-1,199$ & $1,16 \mathrm{E}-05$ & $5,31 \mathrm{E}-05$ & 2,609 \\
\hline 14328 RAB31 & $-1,202$ & $4,99 \mathrm{E}-11$ & $1,05 \mathrm{E}-09$ & 14,907 \\
\hline 6670 GFPT2 & $-1,202$ & $3,86 \mathrm{E}-09$ & $4,62 \mathrm{E}-08$ & 10,558 \\
\hline 11692 MYH2 & $-1,202$ & $1,29 \mathrm{E}-03$ & $3,55 \mathrm{E}-03$ & $-1,972$ \\
\hline 5072 EDN2 & $-1,203$ & 9,88E-07 & $5,93 \mathrm{E}-06$ & 5,038 \\
\hline 10794 MAGI2 & $-1,204$ & $2,41 \mathrm{E}-10$ & $4,16 \mathrm{E}-09$ & 13,330 \\
\hline 11652 MVP & $-1,204$ & $4,18 \mathrm{E}-06$ & $2,14 \mathrm{E}-05$ & 3,611 \\
\hline 3363 CEBPB & $-1,207$ & $3,72 \mathrm{E}-06$ & $1,93 \mathrm{E}-05$ & 3,726 \\
\hline 3832 COL4A6 & $-1,208$ & $3,82 \mathrm{E}-15$ & $3,47 \mathrm{E}-13$ & 24,387 \\
\hline $8461 \mathrm{KCNJ} 8$ & $-1,208$ & $3,13 \mathrm{E}-10$ & $5,18 \mathrm{E}-09$ & 13,068 \\
\hline 15302 SAT2 & $-1,208$ & $3,30 \mathrm{E}-16$ & $4,39 \mathrm{E}-14$ & 26,829 \\
\hline 2306 C2orf40 & $-1,209$ & $3,06 \mathrm{E}-09$ & $3,79 \mathrm{E}-08$ & 10,792 \\
\hline 1082 ATF4 & $-1,210$ & $6,82 \mathrm{E}-08$ & $5,65 \mathrm{E}-07$ & 7,693 \\
\hline 11706 MYL6B & $-1,212$ & $3,27 \mathrm{E}-08$ & $2,95 \mathrm{E}-07$ & 8,427 \\
\hline 10544 LRFN5 & $-1,212$ & 4,57E-09 & $5,36 \mathrm{E}-08$ & 10,389 \\
\hline
\end{tabular}




\begin{tabular}{|c|c|c|c|c|}
\hline 4206 CUTC & $-1,212$ & $7,60 \mathrm{E}-13$ & 2,99E-11 & 19,096 \\
\hline 6535 GALNT5 & $-1,214$ & $1,31 \mathrm{E}-15$ & $1,44 \mathrm{E}-13$ & 25,450 \\
\hline 16371 SOCS2 & $-1,214$ & $1,56 \mathrm{E}-05$ & $6,94 \mathrm{E}-05$ & 2,312 \\
\hline $2701 \mathrm{CA} 3$ & $-1,215$ & $3,13 \mathrm{E}-03$ & 7,73E-03 & $-2,811$ \\
\hline $3793 \mathrm{COCH}$ & $-1,217$ & $1,80 \mathrm{E}-08$ & $1,77 \mathrm{E}-07$ & 9,020 \\
\hline 11412 MRAP2 & $-1,219$ & $8,37 \mathrm{E}-13$ & $3,24 \mathrm{E}-11$ & 18,998 \\
\hline 18999 ZВТВ20 & $-1,220$ & $5,45 \mathrm{E}-07$ & $3,52 \mathrm{E}-06$ & 5,628 \\
\hline 5019 DZIP1 & $-1,220$ & $5,34 \mathrm{E}-12$ & $1,55 \mathrm{E}-10$ & 17,144 \\
\hline 6786 GLRX & $-1,221$ & $3,11 \mathrm{E}-05$ & $1,29 \mathrm{E}-04$ & 1,636 \\
\hline 6384 FRY & $-1,222$ & $5,14 \mathrm{E}-08$ & 4,39E-07 & 7,975 \\
\hline 3663 CLEC4E & $-1,223$ & $1,20 \mathrm{E}-06$ & 7,07E-06 & 4,843 \\
\hline 8904 KRT19 & $-1,224$ & $2,21 \mathrm{E}-04$ & 7,40E-04 & $-0,274$ \\
\hline 18624 VIP & $-1,224$ & $1,01 \mathrm{E}-04$ & $3,69 \mathrm{E}-04$ & 0,482 \\
\hline $11302 \mathrm{MME}$ & $-1,226$ & $1,64 \mathrm{E}-04$ & $5,68 \mathrm{E}-04$ & 0,013 \\
\hline 3676 CLIC6 & $-1,228$ & $1,10 \mathrm{E}-11$ & $2,84 \mathrm{E}-10$ & 16,423 \\
\hline 8580 KIAA0512 & $-1,228$ & $1,08 \mathrm{E}-16$ & $1,71 \mathrm{E}-14$ & 27,945 \\
\hline 14438 RAP1A & $-1,230$ & $6,11 \mathrm{E}-07$ & $3,89 \mathrm{E}-06$ & 5,515 \\
\hline 1953 C17orf91 & $-1,232$ & $1,45 \mathrm{E}-11$ & $3,59 \mathrm{E}-10$ & 16,144 \\
\hline 16523 SPOCK3 & $-1,233$ & $1,69 \mathrm{E}-10$ & 3,04E-09 & 13,684 \\
\hline 6004 FGFR2 & $-1,235$ & $8,09 \mathrm{E}-22$ & $6,13 \mathrm{E}-19$ & 39,593 \\
\hline 4717 DLL1 & $-1,237$ & $1,26 \mathrm{E}-07$ & $9,62 \mathrm{E}-07$ & 7,084 \\
\hline 14150 PTGER2 & $-1,238$ & $1,00 \mathrm{E}-07$ & $7,86 \mathrm{E}-07$ & 7,312 \\
\hline 10947 MBNL2 & $-1,239$ & $1,66 \mathrm{E}-10$ & 3,00E-09 & 13,701 \\
\hline 13777 PPP1R1A & $-1,240$ & 8,94E-09 & 9,70E-08 & 9,720 \\
\hline 17908 TRIM2 & $-1,240$ & $2,79 \mathrm{E}-10$ & 4,74E-09 & 13,184 \\
\hline 8889 KRT17 & $-1,240$ & 8,09E-09 & $8,93 \mathrm{E}-08$ & 9,819 \\
\hline 7223 GULP1 & $-1,240$ & $5,18 \mathrm{E}-09$ & $6,00 \mathrm{E}-08$ & 10,264 \\
\hline 12082 NHS & $-1,241$ & $3,57 \mathrm{E}-16$ & $4,66 \mathrm{E}-14$ & 26,750 \\
\hline 4108 CSRP1 & $-1,241$ & $8,41 \mathrm{E}-11$ & $1,66 \mathrm{E}-09$ & 14,384 \\
\hline 17286 THBD & $-1,242$ & $2,17 \mathrm{E}-07$ & $1,55 \mathrm{E}-06$ & 6,543 \\
\hline 4773 DNAJB4 & $-1,245$ & 7,29E-08 & 5,98E-07 & 7,628 \\
\hline 12377 NUAK1 & $-1,250$ & $6,98 \mathrm{E}-14$ & $4,11 \mathrm{E}-12$ & 21,483 \\
\hline 13769 PPP1R14A & $-1,250$ & $4,01 \mathrm{E}-11$ & $8,69 \mathrm{E}-10$ & 15,125 \\
\hline 13061 PDE5A & $-1,250$ & $4,19 \mathrm{E}-14$ & $2,70 \mathrm{E}-12$ & 21,994 \\
\hline 13436 PLCB1 & $-1,250$ & $7,47 \mathrm{E}-12$ & $2,06 \mathrm{E}-10$ & 16,807 \\
\hline 6023 FIBIN & $-1,251$ & 2,18E-09 & 2,83E-08 & 11,130 \\
\hline 13674 POTEF & $-1,252$ & $1,02 \mathrm{E}-07$ & $8,01 \mathrm{E}-07$ & 7,293 \\
\hline 9055 LATS2 & $-1,253$ & $8,97 \mathrm{E}-10$ & $1,30 \mathrm{E}-08$ & 12,016 \\
\hline 17820 TPM1 & $-1,255$ & $2,06 \mathrm{E}-12$ & $6,82 \mathrm{E}-11$ & 18,099 \\
\hline 5308 EPAS 1 & $-1,255$ & $2,60 \mathrm{E}-12$ & $8,31 \mathrm{E}-11$ & 17,863 \\
\hline 18576 VAMP8 & $-1,255$ & $1,11 \mathrm{E}-05$ & $5,13 \mathrm{E}-05$ & 2,646 \\
\hline 11685 MYF6 & $-1,256$ & $3,53 \mathrm{E}-04$ & $1,12 \mathrm{E}-03$ & $-0,727$ \\
\hline 2830 CARD10 & $-1,257$ & 2,29E-12 & 7,50E-11 & 17,990 \\
\hline
\end{tabular}




\begin{tabular}{|c|c|c|c|c|}
\hline 216 ACTN2 & $-1,260$ & $7,48 \mathrm{E}-04$ & $2,18 \mathrm{E}-03$ & $-1,450$ \\
\hline 8368 JPH2 & $-1,264$ & $2,05 \mathrm{E}-18$ & $6,38 \mathrm{E}-16$ & 31,877 \\
\hline 5312 EPB41L3 & $-1,264$ & $5,81 \mathrm{E}-10$ & $8,95 \mathrm{E}-09$ & 12,451 \\
\hline 1874 C16orf45 & $-1,267$ & $1,40 \mathrm{E}-12$ & $4,99 \mathrm{E}-11$ & 18,486 \\
\hline 3507 CHMP4A & $-1,269$ & $4,50 \mathrm{E}-09$ & $5,29 \mathrm{E}-08$ & 10,405 \\
\hline 2290 C2orf12 & $-1,270$ & $3,15 \mathrm{E}-09$ & $3,88 \mathrm{E}-08$ & 10,762 \\
\hline 8479 KCNMB 1 & $-1,271$ & 7,91E-16 & $9,27 \mathrm{E}-14$ & 25,957 \\
\hline 15833 SLC12A2 & $-1,271$ & 4,64E-07 & $3,05 \mathrm{E}-06$ & 5,787 \\
\hline 4684 DKFZp66 & $-1,272$ & $7,98 \mathrm{E}-16$ & $9,27 \mathrm{E}-14$ & 25,948 \\
\hline 11705 MYL6 & $-1,274$ & $1,56 \mathrm{E}-12$ & $5,44 \mathrm{E}-11$ & 18,378 \\
\hline 11512 MRVI1 & $-1,280$ & $4,45 \mathrm{E}-07$ & 2,94E-06 & 5,828 \\
\hline 16062 SLC39A2 & $-1,280$ & $1,44 \mathrm{E}-06$ & $8,29 \mathrm{E}-06$ & 4,668 \\
\hline 13923 PRKD1 & $-1,281$ & $6,54 \mathrm{E}-10$ & 9,88E-09 & 12,332 \\
\hline 7244 H1F0 & $-1,281$ & $1,72 \mathrm{E}-05$ & $7,58 \mathrm{E}-05$ & 2,219 \\
\hline 17560 TMEM204 & $-1,283$ & 3,11E-09 & $3,84 \mathrm{E}-08$ & 10,774 \\
\hline 7664 HOPX & $-1,283$ & $8,42 \mathrm{E}-06$ & $4,00 \mathrm{E}-05$ & 2,921 \\
\hline 1415 BEX2 & $-1,284$ & $7,45 \mathrm{E}-04$ & $2,17 \mathrm{E}-03$ & $-1,446$ \\
\hline 958 ARMCX1 & $-1,286$ & $3,14 \mathrm{E}-09$ & $3,87 \mathrm{E}-08$ & 10,765 \\
\hline 13296 PID1 & $-1,287$ & $3,28 \mathrm{E}-10$ & 5,39E-09 & 13,022 \\
\hline 11746 MYOM2 & $-1,287$ & $5,16 \mathrm{E}-10$ & $8,06 \mathrm{E}-09$ & 12,569 \\
\hline 16222 SMARCD & $-1,289$ & $1,01 \mathrm{E}-07$ & 7,93E-07 & 7,303 \\
\hline 11542 MSRB3 & $-1,290$ & $2,31 \mathrm{E}-21$ & $1,38 \mathrm{E}-18$ & 38,566 \\
\hline 4678 DKFZP56 & $-1,294$ & $6,77 \mathrm{E}-12$ & $1,89 \mathrm{E}-10$ & 16,906 \\
\hline 11929 NDRG2 & $-1,295$ & $1,71 \mathrm{E}-12$ & $5,90 \mathrm{E}-11$ & 18,281 \\
\hline 6496 GABRP & $-1,295$ & 2,90E-09 & $3,62 \mathrm{E}-08$ & 10,845 \\
\hline 4329 CYP3A5 & $-1,296$ & $3,04 \mathrm{E}-08$ & $2,78 \mathrm{E}-07$ & 8,499 \\
\hline 3625 CLDN1 & $-1,297$ & $6,68 \mathrm{E}-11$ & $1,35 \mathrm{E}-09$ & 14,615 \\
\hline 1359 ВCHE & $-1,300$ & $2,00 \mathrm{E}-03$ & $5,20 \mathrm{E}-03$ & $-2,385$ \\
\hline 3199 CD59 & $-1,300$ & $2,27 \mathrm{E}-16$ & $3,20 \mathrm{E}-14$ & 27,200 \\
\hline 3248 CDC37L1 & $-1,300$ & $1,66 \mathrm{E}-15$ & $1,74 \mathrm{E}-13$ & 25,216 \\
\hline 17026 TBC1D1 & $-1,301$ & $2,25 \mathrm{E}-14$ & $1,59 \mathrm{E}-12$ & 22,613 \\
\hline 18549 UST & $-1,305$ & $1,52 \mathrm{E}-18$ & $4,98 \mathrm{E}-16$ & 32,176 \\
\hline 5537 FAM107A & $-1,306$ & $7,95 \mathrm{E}-07$ & $4,88 \mathrm{E}-06$ & 5,253 \\
\hline 503 ALDH2 & $-1,311$ & 7,53E-08 & $6,16 \mathrm{E}-07$ & 7,595 \\
\hline 9874 LOC3468ع & $-1,312$ & $7,37 \mathrm{E}-13$ & $2,93 \mathrm{E}-11$ & 19,125 \\
\hline 3391 CENPN & $-1,312$ & $8,26 \mathrm{E}-07$ & $5,05 \mathrm{E}-06$ & 5,215 \\
\hline 9090 LCN2 & $-1,317$ & $1,78 \mathrm{E}-03$ & $4,71 \mathrm{E}-03$ & $-2,278$ \\
\hline 16845 SUSD5 & $-1,317$ & $9,86 \mathrm{E}-12$ & $2,59 \mathrm{E}-10$ & 16,530 \\
\hline 14024 PRRX1 & $-1,319$ & $3,07 \mathrm{E}-16$ & $4,17 \mathrm{E}-14$ & 26,901 \\
\hline $8417 \mathrm{KCNB} 1$ & $-1,320$ & $1,23 \mathrm{E}-11$ & $3,12 \mathrm{E}-10$ & 16,306 \\
\hline 13659 PON3 & $-1,321$ & $1,16 \mathrm{E}-05$ & $5,32 \mathrm{E}-05$ & 2,607 \\
\hline 2800 CAND2 & $-1,327$ & $2,87 \mathrm{E}-15$ & $2,75 \mathrm{E}-13$ & 24,672 \\
\hline 19317 ZNF334 & $-1,327$ & $1,79 \mathrm{E}-12$ & $6,10 \mathrm{E}-11$ & 18,237 \\
\hline
\end{tabular}




\begin{tabular}{|c|c|c|c|c|}
\hline 4363 D4S234E & $-1,328$ & $1,75 \mathrm{E}-08$ & $1,72 \mathrm{E}-07$ & 9,050 \\
\hline 14533 RBM3 & $-1,331$ & $1,87 \mathrm{E}-05$ & $8,15 \mathrm{E}-05$ & 2,136 \\
\hline 6929 GPC4 & $-1,332$ & $1,40 \mathrm{E}-14$ & $1,03 \mathrm{E}-12$ & 23,089 \\
\hline $3421 \mathrm{CES} 1$ & $-1,333$ & $3,57 \mathrm{E}-10$ & 5,79E-09 & 12,937 \\
\hline 17666 TMPRSS4 & $-1,334$ & $1,25 \mathrm{E}-05$ & $5,68 \mathrm{E}-05$ & 2,534 \\
\hline 4428 DCN & $-1,334$ & $3,81 \mathrm{E}-04$ & 1,19E-03 & $-0,801$ \\
\hline 447 AJAP1 & $-1,335$ & $2,52 \mathrm{E}-14$ & $1,75 \mathrm{E}-12$ & 22,503 \\
\hline 13148 PERP & $-1,335$ & 4,61E-09 & 5,39E-08 & 10,380 \\
\hline 3279 CDH11 & $-1,337$ & $2,36 \mathrm{E}-08$ & $2,22 \mathrm{E}-07$ & 8,753 \\
\hline 7041 GPR87 & $-1,338$ & $1,26 \mathrm{E}-17$ & $2,93 \mathrm{E}-15$ & 30,073 \\
\hline 4734 DMKN & $-1,339$ & $5,91 \mathrm{E}-12$ & $1,69 \mathrm{E}-10$ & 17,042 \\
\hline 11660 MXRA7 & $-1,339$ & $1,20 \mathrm{E}-20$ & $5,64 \mathrm{E}-18$ & 36,949 \\
\hline 16897 SYT1 & $-1,340$ & $9,64 \mathrm{E}-11$ & 1,87E-09 & 14,247 \\
\hline 11708 MYL9 & $-1,341$ & $4,34 \mathrm{E}-12$ & $1,28 \mathrm{E}-10$ & 17,352 \\
\hline 4785 DNAJC15 & $-1,345$ & $2,69 \mathrm{E}-12$ & $8,54 \mathrm{E}-11$ & 17,830 \\
\hline 3071 CCL23 & $-1,346$ & $4,09 \mathrm{E}-10$ & 6,53E-09 & 12,803 \\
\hline 3364 CEBPD & $-1,347$ & $1,21 \mathrm{E}-05$ & $5,52 \mathrm{E}-05$ & 2,565 \\
\hline 4693 DKK1 & $-1,349$ & 4,81E-09 & $5,60 \mathrm{E}-08$ & 10,339 \\
\hline 3446 CFTR & $-1,349$ & $4,98 \mathrm{E}-05$ & $1,96 \mathrm{E}-04$ & 1,177 \\
\hline 18689 VTCN1 & $-1,350$ & 7,42E-08 & $6,08 \mathrm{E}-07$ & 7,610 \\
\hline 8289 ITGB1BP $_{2}^{\prime}$ & $-1,351$ & $2,12 \mathrm{E}-11$ & $5,02 \mathrm{E}-10$ & 15,762 \\
\hline 11560 МТ1M & $-1,351$ & $1,90 \mathrm{E}-04$ & $6,49 \mathrm{E}-04$ & $-0,129$ \\
\hline 9442 LOC1001? & $-1,351$ & $1,34 \mathrm{E}-02$ & $2,75 \mathrm{E}-02$ & $-4,160$ \\
\hline 7703 HOXD13 & $-1,352$ & $2,93 \mathrm{E}-17$ & $5,95 \mathrm{E}-15$ & 29,238 \\
\hline 9858 LOC34123 & $-1,354$ & $3,65 \mathrm{E}-10$ & 5,90E-09 & 12,916 \\
\hline 9963 LOC39995 & $-1,357$ & $4,86 \mathrm{E}-17$ & $8,71 \mathrm{E}-15$ & 28,734 \\
\hline 5000 DYNC1I1 & $-1,358$ & $9,20 \mathrm{E}-15$ & $7,36 \mathrm{E}-13$ & 23,508 \\
\hline 15125 RRAD & $-1,359$ & $1,43 \mathrm{E}-08$ & $1,45 \mathrm{E}-07$ & 9,250 \\
\hline 12256 NPNT & $-1,360$ & $3,82 \mathrm{E}-05$ & $1,54 \mathrm{E}-04$ & 1,436 \\
\hline 1143 ATP2B4 & $-1,363$ & $1,53 \mathrm{E}-17$ & $3,39 \mathrm{E}-15$ & 29,882 \\
\hline 15384 SCPEP1 & $-1,364$ & $3,80 \mathrm{E}-12$ & $1,14 \mathrm{E}-10$ & 17,485 \\
\hline 14595 RDH10 & $-1,365$ & $9,26 \mathrm{E}-08$ & 7,33E-07 & 7,389 \\
\hline 14133 РТВР2 & $-1,366$ & $5,08 \mathrm{E}-13$ & $2,14 \mathrm{E}-11$ & 19,498 \\
\hline 7800 HSPA5 & $-1,366$ & $5,91 \mathrm{E}-07$ & $3,78 \mathrm{E}-06$ & 5,548 \\
\hline 6255 FLNC & $-1,368$ & $3,48 \mathrm{E}-15$ & $3,20 \mathrm{E}-13$ & 24,480 \\
\hline 13013 PCP4L1 & $-1,368$ & $8,07 \mathrm{E}-05$ & $3,02 \mathrm{E}-04$ & 0,705 \\
\hline 13760 PРP1CB & $-1,372$ & $3,18 \mathrm{E}-11$ & $7,07 \mathrm{E}-10$ & 15,358 \\
\hline 17116 TCEAL7 & $-1,376$ & $1,14 \mathrm{E}-18$ & $3,86 \mathrm{E}-16$ & 32,462 \\
\hline 725 ANXA3 & $-1,376$ & $1,67 \mathrm{E}-06$ & $9,44 \mathrm{E}-06$ & 4,521 \\
\hline 10672 LTBP1 & $-1,377$ & $2,04 \mathrm{E}-08$ & 1,97E-07 & 8,899 \\
\hline 10829 MAOA & $-1,378$ & 7,81E-06 & 3,73E-05 & 2,995 \\
\hline 2650 C9orf3 & $-1,378$ & $3,24 \mathrm{E}-15$ & $3,00 \mathrm{E}-13$ & 24,550 \\
\hline 5411 ESR1 & $-1,379$ & 3,91E-15 & $3,51 \mathrm{E}-13$ & 24,362 \\
\hline
\end{tabular}




\begin{tabular}{|c|c|c|c|c|}
\hline 14813 RNASE4 & $-1,380$ & $2,26 \mathrm{E}-06$ & $1,24 \mathrm{E}-05$ & 4,219 \\
\hline 14045 PRUNE2 & $-1,380$ & $3,29 \mathrm{E}-05$ & $1,35 \mathrm{E}-04$ & 1,583 \\
\hline 6772 GLIPR2 & $-1,380$ & $4,40 \mathrm{E}-06$ & $2,24 \mathrm{E}-05$ & 3,562 \\
\hline 11115 MFN2 & $-1,382$ & $1,92 \mathrm{E}-14$ & $1,38 \mathrm{E}-12$ & 22,772 \\
\hline 12774 P2RY2 & $-1,383$ & $3,15 \mathrm{E}-14$ & $2,12 \mathrm{E}-12$ & 22,278 \\
\hline 6364 FREQ & $-1,384$ & $6,67 E-19$ & $2,44 \mathrm{E}-16$ & 32,988 \\
\hline 4654 DIXDC1 & $-1,387$ & $1,14 \mathrm{E}-16$ & $1,78 \mathrm{E}-14$ & 27,889 \\
\hline 6966 GPR124 & $-1,392$ & $1,78 \mathrm{E}-16$ & $2,65 \mathrm{E}-14$ & 27,441 \\
\hline 11170 MGC2410 & $-1,393$ & $2,02 \mathrm{E}-12$ & $6,75 \mathrm{E}-11$ & 18,116 \\
\hline 6514 GAL & $-1,398$ & $3,85 \mathrm{E}-03$ & $9,25 \mathrm{E}-03$ & $-3,004$ \\
\hline 7973 IGFBP5 & $-1,399$ & $6,43 \mathrm{E}-12$ & $1,81 \mathrm{E}-10$ & 16,958 \\
\hline 1317 BASP1 & $-1,401$ & 2,89E-04 & $9,37 \mathrm{E}-04$ & $-0,534$ \\
\hline 5096 EFEMP1 & $-1,401$ & $1,45 \mathrm{E}-05$ & $6,48 \mathrm{E}-05$ & 2,387 \\
\hline 6654 GEM & $-1,401$ & $3,14 \mathrm{E}-06$ & $1,66 \mathrm{E}-05$ & 3,894 \\
\hline 16390 SORD & $-1,403$ & 2,32E-02 & $4,43 \mathrm{E}-02$ & $-4,657$ \\
\hline 7156 GSTA5 & $-1,411$ & $1,01 \mathrm{E}-09$ & $1,44 \mathrm{E}-08$ & 11,900 \\
\hline 4273 CYB5R2 & $-1,413$ & $9,80 \mathrm{E}-10$ & $1,40 \mathrm{E}-08$ & 11,928 \\
\hline 13891 PRICKLE' & $-1,415$ & $3,98 \mathrm{E}-17$ & $7,61 \mathrm{E}-15$ & 28,934 \\
\hline 5884 FBXO30 & $-1,417$ & $2,99 \mathrm{E}-17$ & $6,01 \mathrm{E}-15$ & 29,218 \\
\hline 321 ADH1C & $-1,417$ & $4,64 \mathrm{E}-07$ & $3,05 \mathrm{E}-06$ & 5,788 \\
\hline 15303 SATB1 & $-1,418$ & $5,91 \mathrm{E}-10$ & $9,06 \mathrm{E}-09$ & 12,435 \\
\hline 4638 DIO3 & $-1,418$ & $1,11 \mathrm{E}-12$ & $4,15 \mathrm{E}-11$ & 18,711 \\
\hline 13949 PRNP & $-1,419$ & $3,20 \mathrm{E}-11$ & $7,10 \mathrm{E}-10$ & 15,351 \\
\hline 6773 GLIS1 & $-1,421$ & $8,15 \mathrm{E}-11$ & $1,61 \mathrm{E}-09$ & 14,416 \\
\hline 3286 CDH19 & $-1,427$ & $5,55 \mathrm{E}-13$ & $2,29 \mathrm{E}-11$ & 19,410 \\
\hline 4963 DUSP1 & $-1,432$ & $1,18 \mathrm{E}-06$ & $6,97 \mathrm{E}-06$ & 4,860 \\
\hline 4809 DNALI1 & $-1,437$ & $2,27 \mathrm{E}-05$ & $9,71 \mathrm{E}-05$ & 1,947 \\
\hline 11061 MEIS2 & $-1,439$ & $9,06 \mathrm{E}-16$ & $1,04 \mathrm{E}-13$ & 25,822 \\
\hline 15888 SLC20A2 & $-1,446$ & $1,22 \mathrm{E}-14$ & $9,27 \mathrm{E}-13$ & 23,224 \\
\hline 16489 SPG20 & $-1,452$ & $5,95 \mathrm{E}-15$ & $5,00 \mathrm{E}-13$ & 23,944 \\
\hline 14921 ROBO1 & $-1,452$ & $8,08 \mathrm{E}-16$ & $9,32 \mathrm{E}-14$ & 25,936 \\
\hline 7030 GPR64 & $-1,453$ & $1,10 \mathrm{E}-04$ & $3,97 \mathrm{E}-04$ & 0,404 \\
\hline 10529 LPP & $-1,456$ & $5,48 \mathrm{E}-13$ & $2,27 \mathrm{E}-11$ & 19,422 \\
\hline 573 AMD1 & $-1,460$ & $1,55 \mathrm{E}-05$ & $6,87 \mathrm{E}-05$ & 2,322 \\
\hline 16751 STOM & $-1,461$ & $5,77 \mathrm{E}-12$ & $1,66 \mathrm{E}-10$ & 17,065 \\
\hline 11108 MFAP4 & $-1,462$ & $3,45 \mathrm{E}-06$ & $1,81 \mathrm{E}-05$ & 3,801 \\
\hline $11556 \mathrm{MT} 1 \mathrm{G}$ & $-1,462$ & $2,15 \mathrm{E}-04$ & $7,22 \mathrm{E}-04$ & $-0,246$ \\
\hline 2060 C1orf133 & $-1,462$ & $6,98 \mathrm{E}-17$ & $1,20 \mathrm{E}-14$ & 28,375 \\
\hline 17699 TNFRSF1: & $-1,465$ & $1,43 \mathrm{E}-07$ & $1,07 \mathrm{E}-06$ & 6,959 \\
\hline $411 \mathrm{AGT}$ & $-1,468$ & $2,60 \mathrm{E}-04$ & $8,53 \mathrm{E}-04$ & $-0,431$ \\
\hline 10689 LXN & $-1,470$ & $1,26 \mathrm{E}-04$ & $4,49 \mathrm{E}-04$ & 0,271 \\
\hline 16194 SLN & $-1,472$ & $2,66 \mathrm{E}-04$ & 8,71E-04 & $-0,453$ \\
\hline 19642 ZNF827 & $-1,473$ & $8,50 \mathrm{E}-05$ & $3,16 \mathrm{E}-04$ & 0,655 \\
\hline
\end{tabular}




\begin{tabular}{|c|c|c|c|c|}
\hline 705 ANO6 & $-1,475$ & $7,84 \mathrm{E}-14$ & $4,54 \mathrm{E}-12$ & 21,368 \\
\hline 8623 KIAA121C & $-1,476$ & 9,83E-09 & $1,05 \mathrm{E}-07$ & 9,625 \\
\hline 14318 RAB23 & $-1,480$ & $2,01 \mathrm{E}-17$ & $4,32 \mathrm{E}-15$ & 29,610 \\
\hline 2612 C8orf84 & $-1,484$ & 9,92E-09 & $1,06 \mathrm{E}-07$ & 9,616 \\
\hline 18020 TSC22D1 & $-1,485$ & 3,83E-09 & $4,59 \mathrm{E}-08$ & 10,566 \\
\hline 11977 NEB & $-1,487$ & $2,25 \mathrm{E}-04$ & $7,51 \mathrm{E}-04$ & $-0,290$ \\
\hline 6767 GLI3 & $-1,487$ & $1,31 \mathrm{E}-16$ & $2,02 \mathrm{E}-14$ & 27,749 \\
\hline 16810 SULF2 & $-1,490$ & $6,09 \mathrm{E}-09$ & $6,94 \mathrm{E}-08$ & 10,103 \\
\hline 8269 ITGA1 & $-1,491$ & $1,87 \mathrm{E}-08$ & $1,82 \mathrm{E}-07$ & 8,982 \\
\hline 8337 JAKMIP1 & $-1,491$ & $4,93 \mathrm{E}-06$ & $2,48 \mathrm{E}-05$ & 3,449 \\
\hline 16286 SNCA & $-1,492$ & $3,26 \mathrm{E}-08$ & $2,95 \mathrm{E}-07$ & 8,429 \\
\hline 6575 GATA3 & $-1,495$ & $3,26 \mathrm{E}-19$ & $1,21 \mathrm{E}-16$ & 33,697 \\
\hline 6447 FYCO1 & $-1,497$ & $2,16 \mathrm{E}-16$ & $3,09 \mathrm{E}-14$ & 27,252 \\
\hline 7700 HOXD10 & $-1,499$ & $1,55 \mathrm{E}-12$ & $5,44 \mathrm{E}-11$ & 18,380 \\
\hline 11554 MT1E & $-1,500$ & $6,51 \mathrm{E}-07$ & $4,11 \mathrm{E}-06$ & 5,452 \\
\hline 18823 WFDC2 & $-1,501$ & $3,63 \mathrm{E}-12$ & $1,10 \mathrm{E}-10$ & 17,529 \\
\hline 10354 LOC72826 & $-1,505$ & $1,87 \mathrm{E}-12$ & $6,31 \mathrm{E}-11$ & 18,196 \\
\hline 13511 PLS3 & $-1,506$ & $3,80 \mathrm{E}-14$ & $2,50 \mathrm{E}-12$ & 22,091 \\
\hline 1284 BACE1 & $-1,510$ & $1,39 \mathrm{E}-14$ & $1,03 \mathrm{E}-12$ & 23,094 \\
\hline 13103 PDPN & $-1,511$ & $1,90 \mathrm{E}-13$ & $9,16 \mathrm{E}-12$ & 20,479 \\
\hline 5582 FAM129A & $-1,513$ & $1,54 \mathrm{E}-07$ & $1,15 \mathrm{E}-06$ & 6,881 \\
\hline 4016 CRISPLD] & $-1,518$ & $1,55 \mathrm{E}-07$ & $1,15 \mathrm{E}-06$ & 6,876 \\
\hline 2906 CBX7 & $-1,521$ & $5,63 \mathrm{E}-16$ & $7,16 \mathrm{E}-14$ & 26,295 \\
\hline 12466 OAT & $-1,522$ & $1,83 \mathrm{E}-09$ & $2,44 \mathrm{E}-08$ & 11,304 \\
\hline 3842 COL8A2 & $-1,523$ & $3,78 \mathrm{E}-13$ & $1,65 \mathrm{E}-11$ & 19,793 \\
\hline 12944 PCDH8 & $-1,528$ & $8,52 \mathrm{E}-07$ & $5,18 \mathrm{E}-06$ & 5,185 \\
\hline 17676 ТМТС1 & $-1,528$ & $2,11 \mathrm{E}-15$ & $2,16 \mathrm{E}-13$ & 24,979 \\
\hline 4881 DPP4 & $-1,530$ & $2,10 \mathrm{E}-07$ & $1,51 \mathrm{E}-06$ & 6,573 \\
\hline 7797 HSPA2 & $-1,531$ & $7,62 \mathrm{E}-07$ & $4,71 \mathrm{E}-06$ & 5,296 \\
\hline 323 ADH5 & $-1,533$ & $1,09 \mathrm{E}-14$ & $8,43 \mathrm{E}-13$ & 23,339 \\
\hline 3190 CD44 & $-1,533$ & $6,44 \mathrm{E}-07$ & 4,07E-06 & 5,462 \\
\hline 14980 RP6-213H & $-1,535$ & $3,10 \mathrm{E}-08$ & $2,82 \mathrm{E}-07$ & 8,479 \\
\hline 9041 LARP6 & $-1,538$ & $1,79 \mathrm{E}-10$ & $3,20 \mathrm{E}-09$ & 13,628 \\
\hline 15557 SERPINB: & $-1,540$ & $4,57 \mathrm{E}-03$ & $1,08 \mathrm{E}-02$ & $-3,165$ \\
\hline 3944 CPM & $-1,542$ & $1,40 \mathrm{E}-09$ & $1,93 \mathrm{E}-08$ & 11,569 \\
\hline 7809 HSPB7 & $-1,543$ & $1,65 \mathrm{E}-14$ & $1,20 \mathrm{E}-12$ & 22,922 \\
\hline 17258 TGFBR2 & $-1,555$ & $1,26 \mathrm{E}-13$ & $6,61 \mathrm{E}-12$ & 20,892 \\
\hline 12826 PAK1IP1 & $-1,555$ & $8,30 \mathrm{E}-05$ & 3,09E-04 & 0,677 \\
\hline 8287 ITGB1 & $-1,562$ & $1,96 \mathrm{E}-08$ & $1,90 \mathrm{E}-07$ & 8,938 \\
\hline 19219 ZNF185 & $-1,564$ & $4,85 \mathrm{E}-10$ & 7,63E-09 & 12,632 \\
\hline 4729 DMBT1 & $-1,567$ & $1,92 \mathrm{E}-03$ & $5,02 \mathrm{E}-03$ & $-2,347$ \\
\hline 14827 RND3 & $-1,567$ & $1,46 \mathrm{E}-11$ & $3,61 \mathrm{E}-10$ & 16,137 \\
\hline 16261 SMTN & $-1,567$ & $1,27 \mathrm{E}-11$ & $3,21 \mathrm{E}-10$ & 16,274 \\
\hline
\end{tabular}




\begin{tabular}{|c|c|c|c|c|}
\hline 3750 CNN3 & $-1,567$ & $1,72 \mathrm{E}-11$ & $4,18 \mathrm{E}-10$ & 15,970 \\
\hline 16257 SMPX & $-1,568$ & $5,52 \mathrm{E}-06$ & $2,75 \mathrm{E}-05$ & 3,336 \\
\hline 6253 FLNA & $-1,570$ & $3,54 \mathrm{E}-13$ & $1,55 \mathrm{E}-11$ & 19,860 \\
\hline 18981 ZAK & $-1,572$ & $7,01 \mathrm{E}-18$ & $1,75 \mathrm{E}-15$ & 30,658 \\
\hline 4102 CSPG4 & $-1,572$ & $2,20 \mathrm{E}-16$ & $3,12 \mathrm{E}-14$ & 27,233 \\
\hline 11552 MT1A & $-1,577$ & $5,77 \mathrm{E}-05$ & 2,24E-04 & 1,032 \\
\hline 2452 C5orf4 & $-1,580$ & $4,18 \mathrm{E}-09$ & 4,94E-08 & 10,480 \\
\hline 17110 TCEAL1 & $-1,587$ & $2,56 \mathrm{E}-13$ & $1,18 \mathrm{E}-11$ & 20,185 \\
\hline 428 AHR & $-1,589$ & $2,21 \mathrm{E}-12$ & $7,28 \mathrm{E}-11$ & 18,026 \\
\hline 9149 LGALS7 & $-1,590$ & $7,84 \mathrm{E}-10$ & $1,15 \mathrm{E}-08$ & 12,151 \\
\hline 547 ALOX15B & $-1,590$ & $6,34 \mathrm{E}-04$ & $1,88 \mathrm{E}-03$ & $-1,290$ \\
\hline 16195 SLPI & $-1,592$ & $4,86 \mathrm{E}-05$ & $1,92 \mathrm{E}-04$ & 1,201 \\
\hline 15551 SERPINB & $-1,592$ & $4,00 \mathrm{E}-11$ & $8,67 \mathrm{E}-10$ & 15,128 \\
\hline 1232 AVPI1 & $-1,594$ & $1,02 \mathrm{E}-15$ & $1,15 \mathrm{E}-13$ & 25,702 \\
\hline 10809 MAMDC2 & $-1,595$ & $1,45 \mathrm{E}-11$ & $3,59 \mathrm{E}-10$ & 16,144 \\
\hline 10522 LPHN2 & $-1,596$ & $7,73 \mathrm{E}-17$ & $1,28 \mathrm{E}-14$ & 28,272 \\
\hline 15666 SGK1 & $-1,600$ & $5,30 \mathrm{E}-03$ & $1,23 \mathrm{E}-02$ & $-3,305$ \\
\hline 15904 SLC22A3 & $-1,601$ & $3,72 \mathrm{E}-08$ & $3,30 \mathrm{E}-07$ & 8,297 \\
\hline 17736 TNNC2 & $-1,602$ & $2,63 \mathrm{E}-05$ & $1,11 \mathrm{E}-04$ & 1,803 \\
\hline 7046 GPRASP1 & $-1,605$ & $1,76 \mathrm{E}-17$ & $3,85 \mathrm{E}-15$ & 29,746 \\
\hline 15497 SEMG2 & $-1,609$ & $3,47 \mathrm{E}-03$ & $8,45 \mathrm{E}-03$ & $-2,906$ \\
\hline 14693 RGN & $-1,615$ & $7,70 \mathrm{E}-18$ & $1,90 \mathrm{E}-15$ & 30,565 \\
\hline 583 AMIGO2 & $-1,616$ & $3,57 \mathrm{E}-12$ & $1,09 \mathrm{E}-10$ & 17,546 \\
\hline 16877 SYNC & $-1,617$ & $7,68 \mathrm{E}-13$ & $3,02 \mathrm{E}-11$ & 19,084 \\
\hline 6667 GFOD1 & $-1,623$ & $6,71 \mathrm{E}-11$ & $1,35 \mathrm{E}-09$ & 14,611 \\
\hline 14611 REEP2 & $-1,626$ & $2,36 \mathrm{E}-16$ & $3,28 \mathrm{E}-14$ & 27,161 \\
\hline 4977 DUSP26 & $-1,632$ & 2,07E-09 & 2,71E-08 & 11,182 \\
\hline 6281 FMOD & $-1,636$ & 4,81E-09 & $5,60 \mathrm{E}-08$ & 10,339 \\
\hline 9612 LOC10014 & $-1,637$ & 2,33E-07 & $1,66 \mathrm{E}-06$ & 6,473 \\
\hline 829 AQP1 & $-1,637$ & $1,00 \mathrm{E}-14$ & $7,92 \mathrm{E}-13$ & 23,421 \\
\hline 17267 TGM4 & $-1,639$ & $3,90 \mathrm{E}-04$ & $1,22 \mathrm{E}-03$ & $-0,822$ \\
\hline 17951 TRIM63 & $-1,641$ & $3,29 \mathrm{E}-06$ & $1,73 \mathrm{E}-05$ & 3,847 \\
\hline $4151 \mathrm{CTGF}$ & $-1,643$ & $7,22 \mathrm{E}-05$ & 2,73E-04 & 0,814 \\
\hline 7065 GPX2 & $-1,644$ & $1,03 \mathrm{E}-11$ & $2,68 \mathrm{E}-10$ & 16,488 \\
\hline 3674 CLIC4 & $-1,646$ & $3,37 \mathrm{E}-16$ & $4,44 \mathrm{E}-14$ & 26,806 \\
\hline 7810 HSPB8 & $-1,654$ & $1,63 \mathrm{E}-15$ & $1,72 \mathrm{E}-13$ & 25,233 \\
\hline 15661 SGCE & $-1,656$ & 2,49E-08 & $2,32 \mathrm{E}-07$ & 8,699 \\
\hline 13125 PDZRN4 & $-1,657$ & $1,13 \mathrm{E}-13$ & $6,06 \mathrm{E}-12$ & 21,006 \\
\hline 11217 MGST1 & $-1,661$ & 4,57E-06 & $2,32 \mathrm{E}-05$ & 3,523 \\
\hline 9360 LOC10012 & $-1,664$ & $1,55 \mathrm{E}-07$ & $1,15 \mathrm{E}-06$ & 6,875 \\
\hline 3827 COL4A2 & $-1,668$ & $1,00 \mathrm{E}-10$ & $1,94 \mathrm{E}-09$ & 14,207 \\
\hline 8091 IL33 & $-1,675$ & $5,37 \mathrm{E}-10$ & 8,33E-09 & 12,529 \\
\hline 14479 RASGRP2 & $-1,677$ & $3,54 \mathrm{E}-18$ & $9,97 \mathrm{E}-16$ & 31,336 \\
\hline
\end{tabular}




\begin{tabular}{|c|c|c|c|c|}
\hline 18074 TSPYL5 & $-1,678$ & $9,15 \mathrm{E}-07$ & $5,53 \mathrm{E}-06$ & 5,114 \\
\hline 6583 GATM & $-1,683$ & $2,69 \mathrm{E}-14$ & $1,85 \mathrm{E}-12$ & 22,435 \\
\hline 13071 PDE8B & $-1,686$ & $6,32 \mathrm{E}-06$ & 3,09E-05 & 3,204 \\
\hline 13097 PDLIM3 & $-1,690$ & $9,41 \mathrm{E}-14$ & $5,26 \mathrm{E}-12$ & 21,184 \\
\hline 15558 SERPINB & $-1,692$ & $1,90 \mathrm{E}-03$ & 4,99E-03 & $-2,340$ \\
\hline 6457 FZD7 & $-1,698$ & $3,72 \mathrm{E}-14$ & $2,46 \mathrm{E}-12$ & 22,112 \\
\hline 6031 FILIP1L & $-1,699$ & $2,98 \mathrm{E}-10$ & 5,00E-09 & 13,118 \\
\hline 17232 TFCP2L1 & $-1,701$ & 2,09E-06 & $1,15 \mathrm{E}-05$ & 4,296 \\
\hline $18 \mathrm{~A} 2 \mathrm{M}$ & $-1,703$ & $1,86 \mathrm{E}-04$ & $6,35 \mathrm{E}-04$ & $-0,108$ \\
\hline 16755 STON1 & $-1,703$ & $4,91 \mathrm{E}-13$ & $2,07 \mathrm{E}-11$ & 19,533 \\
\hline 4373 DACT3 & $-1,705$ & $3,48 \mathrm{E}-21$ & $1,96 \mathrm{E}-18$ & 38,165 \\
\hline 15239 S100P & $-1,714$ & $3,72 \mathrm{E}-03$ & 8,98E-03 & $-2,974$ \\
\hline 6935 GPER & $-1,715$ & $8,15 \mathrm{E}-07$ & 4,99E-06 & 5,228 \\
\hline 7757 HSD11B1 & $-1,716$ & $3,34 \mathrm{E}-05$ & $1,37 \mathrm{E}-04$ & 1,568 \\
\hline 70 ABCC3 & $-1,718$ & $1,13 \mathrm{E}-11$ & $2,91 \mathrm{E}-10$ & 16,390 \\
\hline 14711 RGS2 & $-1,721$ & $6,03 \mathrm{E}-06$ & 2,97E-05 & 3,250 \\
\hline 18817 WFDC1 & $-1,722$ & $2,90 \mathrm{E}-12$ & $9,10 \mathrm{E}-11$ & 17,755 \\
\hline 7153 GSTA2 & $-1,723$ & $2,08 \mathrm{E}-09$ & $2,73 \mathrm{E}-08$ & 11,174 \\
\hline 11557 МТ1Н & $-1,724$ & $1,54 \mathrm{E}-05$ & $6,84 \mathrm{E}-05$ & 2,329 \\
\hline 18058 TSPAN2 & $-1,724$ & $1,20 \mathrm{E}-22$ & $1,18 \mathrm{E}-19$ & 41,455 \\
\hline 5957 FERMT2 & $-1,726$ & $2,59 \mathrm{E}-17$ & $5,43 \mathrm{E}-15$ & 29,361 \\
\hline 16191 SLMAP & $-1,728$ & 9,89E-17 & $1,59 \mathrm{E}-14$ & 28,028 \\
\hline 14224 PTRF & $-1,732$ & $8,92 \mathrm{E}-21$ & $4,40 \mathrm{E}-18$ & 37,241 \\
\hline $11743 \mathrm{MYOF}$ & $-1,732$ & $9,36 \mathrm{E}-18$ & $2,22 \mathrm{E}-15$ & 30,371 \\
\hline 13765 PPP1R12E & $-1,740$ & $1,13 \mathrm{E}-19$ & $4,64 \mathrm{E}-17$ & 34,743 \\
\hline 15584 SETBP1 & $-1,744$ & $6,52 \mathrm{E}-12$ & $1,83 \mathrm{E}-10$ & 16,944 \\
\hline 13098 PDLIM4 & $-1,748$ & $3,24 \mathrm{E}-15$ & $3,00 \mathrm{E}-13$ & 24,552 \\
\hline 4196 CUGBP2 & $-1,750$ & $1,37 \mathrm{E}-21$ & $9,34 \mathrm{E}-19$ & 39,075 \\
\hline 8787 KLF9 & $-1,755$ & $9,80 \mathrm{E}-10$ & $1,40 \mathrm{E}-08$ & 11,928 \\
\hline 14638 RERG & $-1,755$ & $2,77 \mathrm{E}-18$ & $8,14 \mathrm{E}-16$ & 31,579 \\
\hline 267 ADAMTS & $-1,760$ & $4,20 \mathrm{E}-08$ & 3,68E-07 & 8,178 \\
\hline 10537 LRCH2 & $-1,762$ & $1,19 \mathrm{E}-14$ & $9,07 \mathrm{E}-13$ & 23,250 \\
\hline 12818 PAGE4 & $-1,764$ & $8,86 \mathrm{E}-08$ & 7,06E-07 & 7,433 \\
\hline 4111 CSRP3 & $-1,768$ & $8,62 \mathrm{E}-04$ & $2,47 \mathrm{E}-03$ & $-1,585$ \\
\hline 17114 TCEAL5 & $-1,768$ & $2,03 \mathrm{E}-12$ & $6,76 \mathrm{E}-11$ & 18,112 \\
\hline 4902 DPYSL3 & $-1,775$ & $8,35 \mathrm{E}-18$ & $2,03 \mathrm{E}-15$ & 30,484 \\
\hline 13492 PLEKHO1 & $-1,776$ & $3,82 \mathrm{E}-15$ & $3,47 \mathrm{E}-13$ & 24,385 \\
\hline 15162 RSPO3 & $-1,778$ & $3,09 \mathrm{E}-15$ & $2,93 \mathrm{E}-13$ & 24,599 \\
\hline 6322 FOXF1 & $-1,780$ & $7,56 \mathrm{E}-14$ & $4,41 \mathrm{E}-12$ & 21,403 \\
\hline 6397 FST & $-1,782$ & $3,35 \mathrm{E}-16$ & $4,43 \mathrm{E}-14$ & 26,813 \\
\hline 18186 TUBB6 & $-1,792$ & $3,92 \mathrm{E}-17$ & $7,57 \mathrm{E}-15$ & 28,950 \\
\hline 3524 CHRDL2 & $-1,804$ & $2,73 \mathrm{E}-08$ & $2,52 \mathrm{E}-07$ & 8,606 \\
\hline 14214 PTPRK & $-1,805$ & 2,07E-09 & 2,71E-08 & 11,183 \\
\hline
\end{tabular}




\begin{tabular}{|c|c|c|c|c|}
\hline 9245 LMCD1 & $-1,807$ & 1,89E-09 & $2,51 \mathrm{E}-08$ & 11,271 \\
\hline 13709 PPAPDC3 & $-1,811$ & $2,10 \mathrm{E}-18$ & $6,38 \mathrm{E}-16$ & 31,852 \\
\hline 13503 PLN & $-1,814$ & $3,82 \mathrm{E}-16$ & $4,95 \mathrm{E}-14$ & 26,682 \\
\hline 14182 PTPLA & $-1,814$ & $4,83 \mathrm{E}-21$ & $2,57 \mathrm{E}-18$ & 37,844 \\
\hline 5476 EYA1 & $-1,819$ & $1,29 \mathrm{E}-20$ & $5,89 \mathrm{E}-18$ & 36,882 \\
\hline 5050 ECHDC1 & $-1,824$ & $7,64 \mathrm{E}-15$ & $6,20 \mathrm{E}-13$ & 23,694 \\
\hline 13664 POPDC2 & $-1,826$ & $9,77 \mathrm{E}-20$ & $4,10 \mathrm{E}-17$ & 34,885 \\
\hline 6340 FOXO1 & $-1,830$ & $1,11 \mathrm{E}-11$ & $2,86 \mathrm{E}-10$ & 16,411 \\
\hline 16524 SPON1 & $-1,830$ & $6,37 \mathrm{E}-08$ & $5,32 \mathrm{E}-07$ & 7,761 \\
\hline 2764 CALD1 & $-1,848$ & $6,36 \mathrm{E}-20$ & $2,73 \mathrm{E}-17$ & 35,308 \\
\hline 8317 ITPR1 & $-1,851$ & $1,53 \mathrm{E}-08$ & $1,54 \mathrm{E}-07$ & 9,185 \\
\hline 9945 LOC3913? & $-1,854$ & $1,24 \mathrm{E}-14$ & $9,35 \mathrm{E}-13$ & 23,212 \\
\hline 12497 ODZ2 & $-1,855$ & $2,36 \mathrm{E}-06$ & $1,28 \mathrm{E}-05$ & 4,174 \\
\hline 13704 PPAP2B & $-1,860$ & $3,13 \mathrm{E}-12$ & $9,69 \mathrm{E}-11$ & 17,679 \\
\hline 13507 PLP1 & $-1,861$ & $2,68 \mathrm{E}-12$ & $8,53 \mathrm{E}-11$ & 17,833 \\
\hline 14455 RARB & $-1,865$ & $6,51 \mathrm{E}-14$ & $3,91 \mathrm{E}-12$ & 21,553 \\
\hline 13785 PPP1R3C & $-1,867$ & 2,07E-08 & 1,99E-07 & 8,880 \\
\hline 2627 C9orf125 & $-1,867$ & $2,52 \mathrm{E}-21$ & $1,46 \mathrm{E}-18$ & 38,483 \\
\hline 5986 FGF2 & $-1,870$ & $4,36 \mathrm{E}-21$ & $2,39 \mathrm{E}-18$ & 37,942 \\
\hline 4044 CRYAB & $-1,871$ & $2,35 \mathrm{E}-08$ & 2,21E-07 & 8,757 \\
\hline 11096 METTL7A & $-1,874$ & $2,14 \mathrm{E}-12$ & $7,04 \mathrm{E}-11$ & 18,061 \\
\hline $11079 \mathrm{MET}$ & $-1,876$ & $1,01 \mathrm{E}-18$ & $3,49 \mathrm{E}-16$ & 32,578 \\
\hline $15225 \mathrm{~S} 100 \mathrm{~A} 14$ & $-1,877$ & $6,84 \mathrm{E}-16$ & $8,33 \mathrm{E}-14$ & 26,102 \\
\hline $17201 \mathrm{TES}$ & $-1,880$ & $3,66 \mathrm{E}-12$ & $1,11 \mathrm{E}-10$ & 17,522 \\
\hline $12265 \mathrm{NPTN}$ & $-1,880$ & $1,76 \mathrm{E}-18$ & $5,69 \mathrm{E}-16$ & 32,028 \\
\hline 8396 KBTBD10 & $-1,889$ & $6,16 \mathrm{E}-07$ & 3,91E-06 & 5,507 \\
\hline 3972 CPXM2 & $-1,890$ & $9,22 \mathrm{E}-11$ & $1,80 \mathrm{E}-09$ & 14,293 \\
\hline 11701 MYL2 & $-1,894$ & $5,50 \mathrm{E}-05$ & $2,15 \mathrm{E}-04$ & 1,080 \\
\hline 7806 HSPB2 & $-1,908$ & $3,97 \mathrm{E}-15$ & $3,54 \mathrm{E}-13$ & 24,348 \\
\hline 13798 PPP2R2B & $-1,909$ & $2,50 \mathrm{E}-14$ & $1,74 \mathrm{E}-12$ & 22,511 \\
\hline $11561 \mathrm{MT} 1 \mathrm{X}$ & $-1,909$ & $3,21 \mathrm{E}-06$ & $1,69 \mathrm{E}-05$ & 3,873 \\
\hline 14610 REEP1 & $-1,910$ & $3,89 \mathrm{E}-15$ & $3,51 \mathrm{E}-13$ & 24,369 \\
\hline 7986 IGJ & $-1,914$ & $6,78 \mathrm{E}-04$ & 1,99E-03 & $-1,355$ \\
\hline 14139 PTCHD1 & $-1,915$ & $2,47 \mathrm{E}-18$ & $7,36 \mathrm{E}-16$ & 31,694 \\
\hline 12292 NR2F1 & $-1,924$ & $8,58 \mathrm{E}-17$ & $1,40 \mathrm{E}-14$ & 28,169 \\
\hline 12839 PAM & $-1,925$ & $5,40 \mathrm{E}-22$ & $4,63 \mathrm{E}-19$ & 39,987 \\
\hline 17239 TFF1 & $-1,929$ & $4,50 \mathrm{E}-06$ & 2,29E-05 & 3,539 \\
\hline 11559 MT1L & $-1,931$ & $3,73 \mathrm{E}-06$ & $1,94 \mathrm{E}-05$ & 3,723 \\
\hline $2981 \mathrm{CCDC} 3$ & $-1,934$ & $8,25 \mathrm{E}-07$ & $5,04 \mathrm{E}-06$ & 5,217 \\
\hline 8383 KANK1 & $-1,945$ & $1,56 \mathrm{E}-15$ & $1,67 \mathrm{E}-13$ & 25,282 \\
\hline 11553 МТ1В & $-1,956$ & 7,00E-06 & $3,38 \mathrm{E}-05$ & 3,102 \\
\hline 9146 LGALS3 & $-1,958$ & $6,41 \mathrm{E}-12$ & $1,81 \mathrm{E}-10$ & 16,961 \\
\hline 6314 FOXC1 & $-1,962$ & $1,18 \mathrm{E}-17$ & $2,78 \mathrm{E}-15$ & 30,138 \\
\hline
\end{tabular}




\begin{tabular}{|c|c|c|c|c|}
\hline 17735 TNNC1 & $-1,965$ & $4,36 \mathrm{E}-06$ & $2,22 \mathrm{E}-05$ & 3,570 \\
\hline 13542 PMP22 & $-1,967$ & $7,49 \mathrm{E}-13$ & $2,96 \mathrm{E}-11$ & 19,110 \\
\hline 1022 ASB2 & $-1,968$ & $2,44 \mathrm{E}-15$ & $2,40 \mathrm{E}-13$ & 24,834 \\
\hline 1395 BDKRB2 & $-1,972$ & $6,26 \mathrm{E}-10$ & $9,52 \mathrm{E}-09$ & 12,377 \\
\hline 18038 TSHZ3 & $-1,980$ & $5,77 \mathrm{E}-16$ & $7,21 \mathrm{E}-14$ & 26,271 \\
\hline 11700 MYL1 & $-1,985$ & $3,70 \mathrm{E}-05$ & $1,50 \mathrm{E}-04$ & 1,467 \\
\hline $3430 \mathrm{CFB}$ & $-1,988$ & $1,47 \mathrm{E}-04$ & $5,15 \mathrm{E}-04$ & 0,121 \\
\hline 880 ARHGAP $^{\prime}$ & $-1,993$ & $2,60 \mathrm{E}-25$ & $4,66 \mathrm{E}-22$ & 47,372 \\
\hline 16384 SORBS1 & $-2,011$ & $2,38 \mathrm{E}-15$ & $2,38 \mathrm{E}-13$ & 24,857 \\
\hline 6594 GBP2 & $-2,021$ & $8,45 \mathrm{E}-15$ & $6,83 \mathrm{E}-13$ & 23,593 \\
\hline 6492 GABRE & $-2,025$ & $2,53 \mathrm{E}-12$ & $8,09 \mathrm{E}-11$ & 17,893 \\
\hline 959 ARMCX2 & $-2,029$ & $2,67 \mathrm{E}-12$ & $8,51 \mathrm{E}-11$ & 17,837 \\
\hline 4524 DEFB1 & $-2,033$ & $3,01 \mathrm{E}-05$ & $1,25 \mathrm{E}-04$ & 1,671 \\
\hline 7887 ID4 & $-2,033$ & $6,66 \mathrm{E}-18$ & $1,73 \mathrm{E}-15$ & 30,708 \\
\hline 11378 MOXD1 & $-2,036$ & $9,04 \mathrm{E}-10$ & $1,31 \mathrm{E}-08$ & 12,008 \\
\hline 3141 CD163 & $-2,041$ & $4,74 \mathrm{E}-05$ & $1,88 \mathrm{E}-04$ & 1,225 \\
\hline 4301 CYP1B1 & $-2,044$ & $1,99 \mathrm{E}-04$ & $6,74 \mathrm{E}-04$ & $-0,170$ \\
\hline 15697 SH3BGR & $-2,057$ & $2,00 \mathrm{E}-19$ & $7,74 \mathrm{E}-17$ & 34,178 \\
\hline 2382 C3orf57 & $-2,063$ & $3,03 \mathrm{E}-10$ & $5,03 \mathrm{E}-09$ & 13,103 \\
\hline 14415 RAI2 & $-2,072$ & 2,51E-09 & $3,21 \mathrm{E}-08$ & 10,987 \\
\hline 11060 MEIS1 & $-2,073$ & $5,78 \mathrm{E}-16$ & $7,21 \mathrm{E}-14$ & 26,270 \\
\hline 15698 SH3BGRL & $-2,076$ & $7,31 \mathrm{E}-17$ & $1,24 \mathrm{E}-14$ & 28,328 \\
\hline 12046 NFIL3 & $-2,078$ & $1,32 \mathrm{E}-12$ & $4,77 \mathrm{E}-11$ & 18,541 \\
\hline 17741 TNNT1 & $-2,086$ & $5,81 \mathrm{E}-04$ & $1,73 \mathrm{E}-03$ & $-1,207$ \\
\hline 3442 CFL2 & $-2,089$ & $1,28 \mathrm{E}-15$ & $1,41 \mathrm{E}-13$ & 25,474 \\
\hline 4333 CYP4B1 & $-2,099$ & $1,27 \mathrm{E}-14$ & $9,53 \mathrm{E}-13$ & 23,190 \\
\hline 13562 PNMA1 & $-2,111$ & $7,31 \mathrm{E}-13$ & 2,91E-11 & 19,134 \\
\hline 4939 DSTN & $-2,112$ & $4,42 \mathrm{E}-14$ & $2,80 \mathrm{E}-12$ & 21,940 \\
\hline $11671 \mathrm{MYBPH}$ & $-2,112$ & 2,61E-05 & $1,10 \mathrm{E}-04$ & 1,809 \\
\hline 17106 TCAP & $-2,125$ & $1,13 \mathrm{E}-03$ & $3,14 \mathrm{E}-03$ & $-1,842$ \\
\hline 13094 PDK4 & $-2,138$ & $1,90 \mathrm{E}-07$ & $1,38 \mathrm{E}-06$ & 6,672 \\
\hline 15861 SLC16A5 & $-2,142$ & $7,05 \mathrm{E}-19$ & $2,53 \mathrm{E}-16$ & 32,934 \\
\hline 13432 PLAT & $-2,142$ & 2,90E-06 & $1,54 \mathrm{E}-05$ & 3,971 \\
\hline 17807 ТР63 & $-2,153$ & $5,52 \mathrm{E}-26$ & $1,82 \mathrm{E}-22$ & 48,850 \\
\hline 10511 LPAR1 & $-2,163$ & $7,94 \mathrm{E}-14$ & $4,58 \mathrm{E}-12$ & 21,354 \\
\hline 3256 CDC42EP & $-2,170$ & $1,29 \mathrm{E}-12$ & $4,67 \mathrm{E}-11$ & 18,563 \\
\hline 11562 MT2A & $-2,176$ & $1,27 \mathrm{E}-05$ & $5,75 \mathrm{E}-05$ & 2,519 \\
\hline 11740 MYOC & $-2,179$ & $2,92 \mathrm{E}-13$ & $1,32 \mathrm{E}-11$ & 20,052 \\
\hline 17112 TCEAL3 & $-2,186$ & $1,38 \mathrm{E}-13$ & $7,09 \mathrm{E}-12$ & 20,804 \\
\hline 2879 CAV1 & $-2,188$ & $9,88 \mathrm{E}-14$ & $5,46 \mathrm{E}-12$ & 21,136 \\
\hline 17115 TCEAL6 & $-2,190$ & $4,69 \mathrm{E}-14$ & $2,94 \mathrm{E}-12$ & 21,880 \\
\hline 16967 TAGLN & $-2,198$ & $2,18 \mathrm{E}-19$ & $8,28 \mathrm{E}-17$ & 34,091 \\
\hline 15387 SCRN1 & $-2,212$ & $5,24 \mathrm{E}-15$ & $4,51 \mathrm{E}-13$ & 24,070 \\
\hline
\end{tabular}




\begin{tabular}{|c|c|c|c|c|}
\hline 16577 SRD5A2 & $-2,219$ & $8,15 \mathrm{E}-19$ & $2,87 \mathrm{E}-16$ & 32,790 \\
\hline 8927 KRT5 & $-2,221$ & $3,26 \mathrm{E}-17$ & $6,48 \mathrm{E}-15$ & 29,133 \\
\hline 7080 GRASP & $-2,223$ & $1,47 \mathrm{E}-17$ & $3,30 \mathrm{E}-15$ & 29,920 \\
\hline 591 AMOTL2 & $-2,225$ & $9,08 \mathrm{E}-12$ & $2,41 \mathrm{E}-10$ & 16,613 \\
\hline $11215 \mathrm{MGP}$ & $-2,232$ & $2,95 \mathrm{E}-10$ & $4,96 \mathrm{E}-09$ & 13,127 \\
\hline 6022 FHOD3 & $-2,243$ & $3,95 \mathrm{E}-14$ & $2,56 \mathrm{E}-12$ & 22,052 \\
\hline 718 ANXA1 & $-2,243$ & $1,27 \mathrm{E}-14$ & $9,54 \mathrm{E}-13$ & 23,185 \\
\hline $17683 \mathrm{TNC}$ & $-2,243$ & $2,67 \mathrm{E}-11$ & $6,06 \mathrm{E}-10$ & 15,533 \\
\hline 730 ANXA8L2 & $-2,246$ & $2,97 \mathrm{E}-12$ & $9,27 \mathrm{E}-11$ & 17,732 \\
\hline 1290 BAG2 & $-2,260$ & $6,88 \mathrm{E}-16$ & $8,33 \mathrm{E}-14$ & 26,095 \\
\hline 17113 TCEAL4 & $-2,262$ & $4,65 \mathrm{E}-17$ & $8,48 \mathrm{E}-15$ & 28,779 \\
\hline 6398 FSTL1 & $-2,262$ & $5,78 \mathrm{E}-18$ & $1,56 \mathrm{E}-15$ & 30,849 \\
\hline 7147 GSN & $-2,264$ & $1,53 \mathrm{E}-14$ & $1,12 \mathrm{E}-12$ & 23,001 \\
\hline 1025 ASB5 & $-2,271$ & $6,25 \mathrm{E}-15$ & $5,19 \mathrm{E}-13$ & 23,894 \\
\hline 4695 DKK3 & $-2,274$ & $6,07 \mathrm{E}-16$ & $7,48 \mathrm{E}-14$ & 26,221 \\
\hline 3931 CPAMD8 & $-2,277$ & 7,99E-11 & $1,58 \mathrm{E}-09$ & 14,436 \\
\hline 14576 RCAN2 & $-2,297$ & $8,63 \mathrm{E}-10$ & $1,26 \mathrm{E}-08$ & 12,055 \\
\hline 7769 HSD17B6 & $-2,319$ & $3,22 \mathrm{E}-06$ & $1,70 \mathrm{E}-05$ & 3,868 \\
\hline 16809 SULF1 & $-2,320$ & $9,77 \mathrm{E}-11$ & $1,89 \mathrm{E}-09$ & 14,234 \\
\hline 8276 ITGA5 & $-2,327$ & $4,08 \mathrm{E}-18$ & $1,13 \mathrm{E}-15$ & 31,194 \\
\hline 11748 MYOT & $-2,330$ & $2,17 \mathrm{E}-13$ & $1,03 \mathrm{E}-11$ & 20,350 \\
\hline 16856 SVIL & $-2,343$ & $3,90 \mathrm{E}-15$ & $3,51 \mathrm{E}-13$ & 24,366 \\
\hline $3935 \mathrm{CPE}$ & $-2,353$ & $8,34 \mathrm{E}-08$ & $6,70 \mathrm{E}-07$ & 7,493 \\
\hline 3916 COX7A1 & $-2,389$ & $7,70 \mathrm{E}-21$ & $3,89 \mathrm{E}-18$ & 37,386 \\
\hline 16708 STAT6 & $-2,395$ & $3,09 \mathrm{E}-13$ & $1,39 \mathrm{E}-11$ & 19,994 \\
\hline 14332 RAB34 & $-2,400$ & $6,01 \mathrm{E}-18$ & $1,60 \mathrm{E}-15$ & 30,810 \\
\hline $2170 \mathrm{C} 1 \mathrm{~S}$ & $-2,408$ & $4,20 \mathrm{E}-11$ & $9,03 \mathrm{E}-10$ & 15,080 \\
\hline 352 ADRA2C & $-2,418$ & $1,60 \mathrm{E}-08$ & $1,60 \mathrm{E}-07$ & 9,140 \\
\hline 15617 SFRP1 & $-2,448$ & $3,21 \mathrm{E}-15$ & $3,00 \mathrm{E}-13$ & 24,559 \\
\hline 6451 FZD10 & $-2,458$ & $7,64 \mathrm{E}-17$ & $1,28 \mathrm{E}-14$ & 28,285 \\
\hline 5919 FCGBP & $-2,470$ & $2,08 \mathrm{E}-08$ & 1,99E-07 & 8,879 \\
\hline 8931 KRT7 & $-2,485$ & $3,38 \mathrm{E}-11$ & $7,45 \mathrm{E}-10$ & 15,297 \\
\hline 734 AOC3 & $-2,488$ & $2,79 \mathrm{E}-24$ & $3,93 \mathrm{E}-21$ & 45,095 \\
\hline $2168 \mathrm{C} 1 \mathrm{R}$ & $-2,511$ & $2,06 \mathrm{E}-12$ & $6,82 \mathrm{E}-11$ & 18,096 \\
\hline 17595 TMEM47 & $-2,515$ & $2,60 \mathrm{E}-15$ & $2,54 \mathrm{E}-13$ & 24,770 \\
\hline 11325 MMP7 & $-2,520$ & $1,34 \mathrm{E}-05$ & $6,07 \mathrm{E}-05$ & 2,460 \\
\hline 11693 MYH3 & $-2,549$ & $1,45 \mathrm{E}-10$ & $2,65 \mathrm{E}-09$ & 13,840 \\
\hline 668 ANKRD3! & $-2,554$ & $1,06 \mathrm{E}-21$ & $7,72 \mathrm{E}-19$ & 39,325 \\
\hline 15545 SERPINA. & $-2,585$ & $1,15 \mathrm{E}-04$ & $4,15 \mathrm{E}-04$ & 0,356 \\
\hline 4330 CYP3A7 & $-2,615$ & $2,96 \mathrm{E}-10$ & 4,97E-09 & 13,125 \\
\hline 407 AGR2 & $-2,621$ & $1,69 \mathrm{E}-08$ & $1,67 \mathrm{E}-07$ & 9,084 \\
\hline 207 ACTC1 & $-2,644$ & $2,07 \mathrm{E}-13$ & $9,82 \mathrm{E}-12$ & 20,398 \\
\hline 10804 MAL & $-2,693$ & $1,63 \mathrm{E}-19$ & $6,45 \mathrm{E}-17$ & 34,378 \\
\hline
\end{tabular}




\begin{tabular}{|c|c|c|c|c|}
\hline 11696 MYH7 & $-2,693$ & $4,12 \mathrm{E}-06$ & $2,11 \mathrm{E}-05$ & 3,625 \\
\hline 4774 DNAJB5 & $-2,696$ & $5,43 \mathrm{E}-21$ & $2,82 \mathrm{E}-18$ & 37,728 \\
\hline 15658 SGCA & $-2,701$ & $6,11 \mathrm{E}-22$ & $5,02 \mathrm{E}-19$ & 39,867 \\
\hline 7974 IGFBP6 & $-2,709$ & $4,36 \mathrm{E}-18$ & $1,19 \mathrm{E}-15$ & 31,129 \\
\hline 9132 LEPREL1 & $-2,721$ & $8,71 \mathrm{E}-14$ & $4,92 \mathrm{E}-12$ & 21,262 \\
\hline 13010 PCOLCE2 & $-2,784$ & $2,47 \mathrm{E}-12$ & $7,95 \mathrm{E}-11$ & 17,915 \\
\hline 17111 TCEAL2 & $-2,876$ & $1,19 \mathrm{E}-19$ & $4,80 \mathrm{E}-17$ & 34,688 \\
\hline 7807 HSPB3 & $-2,893$ & $1,39 \mathrm{E}-08$ & $1,41 \mathrm{E}-07$ & 9,282 \\
\hline 6260 FLRT3 & $-2,915$ & $1,08 \mathrm{E}-26$ & $7,12 \mathrm{E}-23$ & 50,395 \\
\hline 8278 ITGA7 & $-2,927$ & $1,10 \mathrm{E}-20$ & $5,28 \mathrm{E}-18$ & 37,037 \\
\hline 16891 SYNPO2 & $-2,930$ & $1,18 \mathrm{E}-25$ & $3,34 \mathrm{E}-22$ & 48,123 \\
\hline 12028 NEXN & $-2,932$ & $5,27 \mathrm{E}-20$ & $2,31 \mathrm{E}-17$ & 35,495 \\
\hline $3601 \mathrm{CKM}$ & $-3,016$ & $1,35 \mathrm{E}-07$ & $1,02 \mathrm{E}-06$ & 7,011 \\
\hline 11989 NEFH & $-3,031$ & $4,63 \mathrm{E}-08$ & $4,01 \mathrm{E}-07$ & 8,080 \\
\hline 15496 SEMG1 & $-3,033$ & $1,08 \mathrm{E}-05$ & $4,99 \mathrm{E}-05$ & 2,676 \\
\hline 737 AOX1 & $-3,038$ & $2,40 \mathrm{E}-11$ & $5,52 \mathrm{E}-10$ & 15,640 \\
\hline 206 ACTBL2 & $-3,048$ & $1,20 \mathrm{E}-18$ & $4,00 \mathrm{E}-16$ & 32,410 \\
\hline 16483 SPEG & $-3,073$ & $1,61 \mathrm{E}-16$ & $2,42 \mathrm{E}-14$ & 27,545 \\
\hline 8909 KRT23 & $-3,081$ & $1,33 \mathrm{E}-16$ & $2,03 \mathrm{E}-14$ & 27,735 \\
\hline 15353 SCHIP1 & $-3,103$ & $1,68 \mathrm{E}-21$ & $1,11 \mathrm{E}-18$ & 38,876 \\
\hline 14457 RARRES 1 & $-3,104$ & $4,80 \mathrm{E}-08$ & $4,14 \mathrm{E}-07$ & 8,044 \\
\hline 17821 TPM2 & $-3,132$ & $4,74 \mathrm{E}-22$ & $4,25 \mathrm{E}-19$ & 40,115 \\
\hline 3518 CHP2 & $-3,169$ & $1,35 \mathrm{E}-14$ & $1,00 \mathrm{E}-12$ & 23,124 \\
\hline 1130 ATP1A2 & $-3,171$ & $7,81 \mathrm{E}-13$ & $3,07 \mathrm{E}-11$ & 19,067 \\
\hline 9105 LDHB & $-3,200$ & $7,48 \mathrm{E}-13$ & $2,96 \mathrm{E}-11$ & 19,111 \\
\hline 11688 MYH11 & $-3,226$ & $3,45 \mathrm{E}-20$ & $1,54 \mathrm{E}-17$ & 35,912 \\
\hline 7166 GSTP1 & $-3,266$ & $1,92 \mathrm{E}-25$ & $3,99 \mathrm{E}-22$ & 47,661 \\
\hline 8885 KRT13 & $-3,289$ & $3,27 \mathrm{E}-13$ & $1,45 \mathrm{E}-11$ & 19,940 \\
\hline 6563 GAS 1 & $-3,310$ & $3,21 \mathrm{E}-13$ & $1,43 \mathrm{E}-11$ & 19,958 \\
\hline 11537 MSMB & $-3,319$ & $1,44 \mathrm{E}-08$ & $1,46 \mathrm{E}-07$ & 9,242 \\
\hline 15232 S100A6 & $-3,330$ & $6,61 \mathrm{E}-16$ & $8,10 \mathrm{E}-14$ & 26,136 \\
\hline 9021 LAMB3 & $-3,370$ & $2,32 \mathrm{E}-23$ & $2,54 \mathrm{E}-20$ & 43,049 \\
\hline 10830 МАOB & $-3,424$ & $1,45 \mathrm{E}-25$ & $3,57 \mathrm{E}-22$ & 47,931 \\
\hline 204 ACTA2 & $-3,471$ & $2,19 \mathrm{E}-24$ & $3,33 \mathrm{E}-21$ & 45,327 \\
\hline 16451 SPARCL1 & $-3,492$ & $1,46 \mathrm{E}-13$ & $7,39 \mathrm{E}-12$ & 20,743 \\
\hline 18844 WISP2 & $-3,507$ & $1,31 \mathrm{E}-14$ & $9,74 \mathrm{E}-13$ & 23,156 \\
\hline 9257 LMOD1 & $-3,552$ & $1,97 \mathrm{E}-23$ & $2,28 \mathrm{E}-20$ & 43,208 \\
\hline 6018 FHL2 & $-3,569$ & $3,42 \mathrm{E}-18$ & $9,77 \mathrm{E}-16$ & 31,370 \\
\hline 203 ACTA1 & $-3,666$ & $1,39 \mathrm{E}-08$ & $1,42 \mathrm{E}-07$ & 9,281 \\
\hline 6017 FHL1 & $-3,689$ & $1,68 \mathrm{E}-23$ & $2,07 \mathrm{E}-20$ & 43,360 \\
\hline 12833 PALLD & $-3,709$ & $1,95 \mathrm{E}-21$ & $1,20 \mathrm{E}-18$ & 38,732 \\
\hline 7884 ID1 & $-3,716$ & $4,26 \mathrm{E}-17$ & $7,93 \mathrm{E}-15$ & 28,865 \\
\hline 11711 MYLK & $-3,719$ & $1,10 \mathrm{E}-21$ & $7,72 \mathrm{E}-19$ & 39,295 \\
\hline
\end{tabular}




\begin{tabular}{ccccc}
17757 TNS4 & $-3,769$ & $5,12 \mathrm{E}-26$ & $1,82 \mathrm{E}-22$ & 48,923 \\
4564 DES & $-3,972$ & $1,35 \mathrm{E}-23$ & $1,77 \mathrm{E}-20$ & 43,575 \\
15228 S100A2 & $-4,303$ & $8,03 \mathrm{E}-23$ & $8,33 \mathrm{E}-20$ & 41,844 \\
16889 SYNM & $-4,307$ & $2,02 \mathrm{E}-25$ & $3,99 \mathrm{E}-22$ & 47,613 \\
12520 OLFM4 & $-4,496$ & $4,65 \mathrm{E}-12$ & $1,37 \mathrm{E}-10$ & 17,283 \\
3748 CNN1 & $-4,905$ & $5,37 \mathrm{E}-29$ & $5,29 \mathrm{E}-25$ & 55,354 \\
8887 KRT15 & $-4,909$ & $4,17 \mathrm{E}-25$ & $6,85 \mathrm{E}-22$ & 46,922 \\
13012 PCP4 & $-5,670$ & $1,21 \mathrm{E}-31$ & $2,38 \mathrm{E}-27$ & 60,887 \\
209 ACTG2 & $-5,945$ & $4,19 \mathrm{E}-26$ & $1,82 \mathrm{E}-22$ & 49,113 \\
\hline
\end{tabular}




Terms Hit p value Gene List

\section{Up-regualted genes}

Leishmania infection

Allograft rejection

Antigen

Type I diabetes mellitus

Asthma

Viral myocarditis

Graft versus host disease

Intestinal immune network for IgA production

Autommune thyroid disease

Systemic lupus erythematosus

Down-regualted genes

Dilated cardiomyopathy

Hypertrophic cardiomyopathy (HCM)

(ARVC)

Cardiac muscle contraction
3,45E-07 HLA-DMA;HLA-DMB;HLA-DPA1;HLA-DRB3;HLA-DRB4;JUN;MARCKSL1

0,000260628 HLA-DMA;HLA-DMB;HLA-DPA1;HLA-DRB3;HLA-DRB4

1,09E-05 HLA-DMA;HLA-DMB;HLA-DPA1;HLA-DRB3;HLA-DRB4;HSP90AB1;HSPA6

5,11E-05 HLA-DMA;HLA-DMB;HLA-DPA1;HLA-DRB3;HLA-DRB4;ICA1

9,30E-05 HLA-DMA;HLA-DMB;HLA-DPA1;HLA-DRB3;HLA-DRB4

0,001069719 HLA-DMA;HLA-DMB;HLA-DPA1;HLA-DRB3;HLA-DRB4

0,000426181 HLA-DMA;HLA-DMB;HLA-DPA1;HLA-DRB3;HLA-DRB4

0,000892171 HLA-DMA;HLA-DMB;HLA-DPA1;HLA-DRB3;HLA-DRB4

0,001288187 HLA-DMA;HLA-DMB;HLA-DPA1;HLA-DRB3;HLA-DRB4

6 0,021962707 HIST1H4E;HLA-DMA;HLA-DMB;HLA-DPA1;HLA-DRB3;HLA-DRB4

19 5,24E-14 ACTC1;CACNA2D1;DES;DMD;ITGA1;ITGA5;ITGA7;ITGA8;ITGB1;LMNA;MYH7;MYL2;PLN;SGCA;TGFB2;TNNC1;TPM1;TPM2;TTN 8,67E-14 ACTC1;CACNA2D1;DES;DMD;ITGA1;ITGA5;ITGA7;ITGA8;ITGB1;LMNA;MYH7;MYL2;SGCA;TGFB2;TNNC1;TPM1;TPM2;TTN

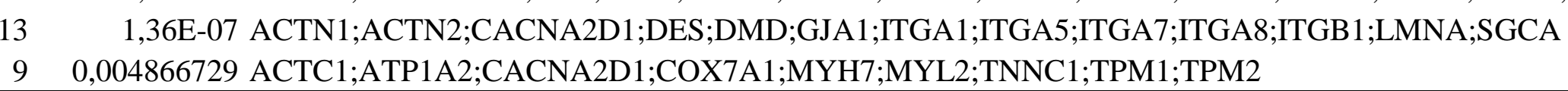




\section{The Gene Ontology (GO) functional enrichment and Kyoto Encyclopedia of Genes and Genomes (KEGG) pathway analysis of}

\section{GO term}

actin filament-based process

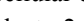

cytoskeletal protein binding

myofibril

contractile fiber

actin binding

contractile fiber par

Pathways

Cardiac muscle contraction

Tight junction

Tic right ventricular cardiomyopathy (ARVC)

Arglcium signal pathway
Cano
Enrichment Score: 80.41

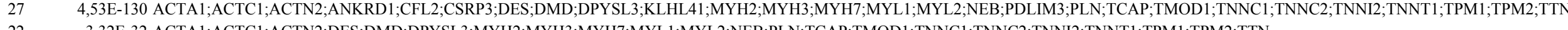
22 3,32E-32 ACTA1;ACTC1;ACTN2;DES;DMD;DPYSL3;MYH2;MYH3;MYH7;MYL1;MYL2;NEB;PLN;TCAP;TMOD1;TNNC1;TNNC2;TNNI2;TNNT1;TPM1;TPM2;TTN

ent Score: 53.87

69E-92 ACTA1;ACTC1;ACTN2;ANKRD1;CFL2;CSRP3;DES;DMD;MYH2;MYH3;MYH7;MYL2;MYOT;NEB;NRAP;:PDLIM3;TCAP;TMOD1;TNNC1;TNNC2;TNN12;TNNT1;TPM1;TPM2;TRIM63;TTN

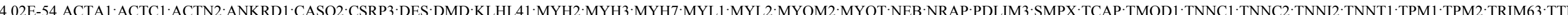

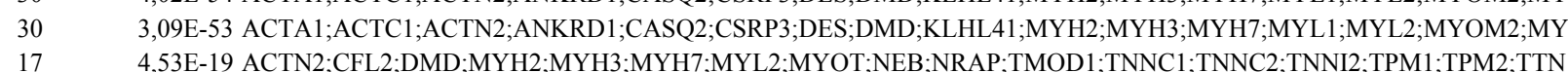

Enrichment Score: 46.94

2,80E-48 ACTA1;ACTC1;ACTN2;ANKRD1;CASQ2;CSRP3;DES;DMD;KLHL41;MYH2;MYH7;MYL1;MYL2;MYOM2;MYOT;NEB;PDLIM3;SMPX;TCAP;TNNC1;TNNC2;TNNI2;TNNT1;TPM1;TPM2;TRIM63;TTN

10 7,19E-13 ACTC1;DES;DMD;MYH7;MYL2;PLN;TNNC1;TPM1;TPM2;TTN

156E-11 ACTC1;DES:DMD;MYH7:MYL2;TNNC1;TPM1;:TPM2;TTN

4,68E-07 ACTC1;MYH7;MYL2;TNNC1;:TPM1;TPM2

0,000148737 ACTN2;MYH2;MYH3;MYH7;MYL2

,

0,003029789 ACTN2,DES;DND

0,03236325 PLN.TNNCl:TN 
Supplemental table 5. Pathways and genes involving in crosstalk pathways

Pathways $\quad$ Gene list

hsa04662:B cell receptor signaling pathway

isa04662:B cell receptor signaling pathway

MAP2K2,JUN,CHP2,PPP3CB,PIK3R1,NFKBIA,PPP3CA

hsa05211:Renal cell carcinoma

hsa04062:Chemokine signaling pathway

CCL3,MAP2K2,JUN,CCL4,PIK3R1,NFKBIA

hsa04810:Regulation of actin cytoskeleton

hsa04910:Insulin signaling pathway

hsa04510:Focal adhesion

hsa05210:Colorectal cancer

hsa04660:T cell receptor signaling pathway

hsa04010:MAPK signaling pathway

hsa05213:Endometrial cancer

sa04370:VEGF signaling pathway

MAP2K2,JUN,MET,EPAS1,PIK3R1,TGFB2,PAK3,RAP1A

CCL3L3,CCL3,GNB2,CCL4,RASGRP2,PLCB1,PIK3R1,CCL23,NFKBIA,RAP1A,GNG4,CXCL1

MAP2K2,FGFR2,MYLK,FGF2,ITGA7,ITGA5,CFL2,GSN,MYL9,PIK3R1,PPP1CB,VCL,BDKRB2,ACTN1,PAK3,ITGA1,ITGB1,ITGA8,MYL2,ACTN2

MAP2K2,MKNK2,FASN,SORBS1,FOXO1,PIK3R1,PPP1CB,PPP1R3C,IRS1,SOCS2

JUN,COL1A1,LAMB3,MYLK,ITGA7,MET,ITGA5,FLNC,COL4A6,CAV1,FLNA,LAMB2,MYL9,BCL2,PIK3R1,TNC,PPP1CB,COL4A2,VCL,LAMC1,ACTN1,PAK3,ITGA1,ITGB1,ITGA8,RAP1A,MYL2,ACTN2,THBS4 JUN,BIRC5,SMAD3,TGFBR2,BCL2,PIK3R1,TGFB2

MAP2K2,JUN,MET,EPAS1,PIK3R1,TGFB2,PAK3,RAP1A

ELK4,MAP2K2,STMN1,JUN,MKNK2,CACNA1D,HSPA6,FGFR2,FGF2,RASGRP2,ZAK,FLNC,CHP2,TGFBR2,FLNA,PPP3CB,TGFB2,CACNA2D1,ATF4,RAP1A,HSPA2,DUSP1,RELB,PPP3CA

MAP2K2,PIK3R1

MAP2K2,CHP2,PPP3CB,PIK3R1,PTGS2,PPP3CA

MAP2K2,JUN,BCL2,PIK3R1,NFKBIA,ATF4,RAP1A,IRS1

CDKN2A,MAP2K2,PIK3R1

CDKN2A,MAP2K2,SMAD3,TGFBR2,PIK3R1,TGFB2,NFKBIA

MAP2K2,PIK3R1

hsa05220:Chronic myeloid leukemia

hsa05221:Acute myeloid leukemia

hsa04012:ErbB signaling pathway

hsa05216:Thyroid cancer

MAP2K2,JUN,PIK3R1,PAK3,AREG

MAP2K2

hsa04150:mTOR signaling pathway

IRS1,CAB39L

hsa05160:Hepatitis C

PPP2R2B,PIK3R1,CLDN1,NFKBIA 
Disease Ontology (DO) annotation of up-regulated differentially expressed genes

DOID:5656 cranial nerve disease

DOID:3526 cerebral infarction

$\mathrm{p}$ value

Hit Gene list

DOID:0050686 organ system cancer

$6,72 \mathrm{E}-04$

5 MMP11,MMP9,ND1,ND6,PHOX2A

4 APOE,F5,FABP1,LGALS2

ABCA3,ABCC5,ADAM8,ADRB2,AP1M2,APLN,APOC1,APOE,AR,B3GAT1,BCAM,BIRC5,CCL3,CC

L4,CCNB1,CDKN2A,COX1,CTAG1A,DLGAP5,ELK4,EPCAM,EZH2,F5,FASN,FGG,HPGD,HPN,HSP

3,NDRG1,PBK, PTTG1,RECQL4,REG1A,SLC19A1,SOX4,STMN1,TK1,TMSB15A,UBE2C,UBE2T Z DHHC11

ABCA3,ABCC5,ADAM8,ADRB2,AP1M2,APLN,APOC1,APOE,AR,B3GAT1,BCAM,BIRC5,CA1,CA

SP10,CCL3,CCL4,CCNB1,CDKN2A,CENPU,COX1,CTAG1A,DLGAP5,ELK4,EPCAM,ESM1,EZH2,F

DOID:162 cancer

DOID:3454 brain infarction

DOID:0060158 acquired metabolic disease

DOID:9351 diabetes mellitus

DOID:14566 disease of cellular proliferation

DOID:3389 Papillon-Lefevre disease

DOID:2151 malignant ovarian surface epithelial-stromal neoplasm

DOID:2152 ovary epithelial cancer

DOID:4001 ovarian carcinoma

DOID:0050013 carbohydrate metabolism disease

DOID:4194 glucose metabolism disease

DOID:0014667 disease of metabolism

DOID:684 hepatocellular carcinoma

DOID:686 liver carcinoma

DOID:4766 embryoma

DOID:5158 pleural cancer

DOID:7474 malignant pleural mesothelioma

DOID:688 embryonal cancer

DOID:0080001 bone disease

DOID:2600 laryngeal carcinoma

DOID:0050687 cell type cancer

DOID:3571 liver cancer

DOID:4450 renal cell carcinoma

DOID:4451 renal carcinoma

DOID:7148 rheumatoid arthritis

DOID:2994 germ cell cancer

DOID:3342 bone inflammation disease

DOID:3093 nervous system cancer

DOID:3302 chordoma

DOID:3303 notochordal cancer

DOID:6204 follicular adenoma
$1,40 \mathrm{E}-03$

61 12,F5,FASN,FGG,HPGD,HPN,HSP90AB1,HSPE1,IDO1,ISG15,ITPR3,JUN,KCNQ1OT1,KIAA0101,L GALS2,MARCKSL1,MCM2,MEL K,MMP11,MMP9,ND3,NDRG1,PBK,PTK6,PTTG1,RECQL4,REG1

A,SLC19A1,SOX4,STMN1,TK1,TMSB15A,UBE2C,UBE2T,ZDHHC11

1,56E-03 4 APOE,F5,FABP1,LGALS2

1,67E-03 20 ADRB2,APLN,APOC1,APOE,AR,CA1,CDKN2A,CDKN3,F5,FAAH,FASN,HLA-DMA,HLA-DMB,ITPR3,MCM2,MMP9,ND1,PCBD1,PVT1,REG1A

1,99E-03 16 ADRB2,APLN,APOC1,APOE,AR,CA1,CDKN2A,F5,HLA-DMA,HLA-DMB,ITPR3,MMP9,ND1,PCBD1,PVT1,REG1A

ABCA3,ABCC5,ADAM8,ADRB2,AP1M2,APLN,APOC1,APOE,AR,B3GAT1,BCAM,BIRC5,CA1,CA

SPI0,CCL3,CCL4,CCNB1,CDKN2A,CEN

2,20E-03 61 12,F5,FASN,FGG,HPGD,HPN,HSP90AB1,HSPE1,IDO1,ISG15,ITPR3,JUN,KCNQ1OT1,KIAA0101,L

GALS2,MARCKSL1,MCM2,MELK,MMP11,MMP9,ND3,NDRG1,PBK,PTK6,PTTG1,RECQL4,REG1 A,SLC19A1,SOX4,STMN1,TK1,TMSB15A,UBE2C,UBE2T,ZDHHC11

$2,28 \mathrm{E}-03 \quad 2$ CCL3,CCL3L3

2,89E-03 5 CDKN2A,EPCAM,MCM2,MMP9,TK1

2,89E-03 5 CDKN2A,EPCAM,MCM2,MMP9,TK1

2,89E-03 5 CDKN2A,EPCAM,MCM2,MMP9,TK1

3,25E-03 16 ADRB2,APLN,APOC1,APOE,AR,CA1,CDKN2A,F5,HLA-DMA,HLA-DMB,ITPR3,MMP9,ND1,PCBD1,PVT1,REG1A

3,25E-03 16 ADRB2,APLN,APOC1,APOE,AR,CA1,CDKN2A,F5,HLA-DMA,HLA-DMB,ITPR3,MMP9,ND1,PCBD1,PVT1,REG1A

3,57E-03 22 ADRB2,APLN,APOC1,APOE,AR,CA1,CDKN2A,CDKN3,F5,FAAH,FASN,HLA-DMA,HLA-DMB,ITPR3,MCM2,MMP9,ND1,ND2,ND3,PCBD1,PVT1,REG1A

3,58E-03 14 APOE,AR,BCAM,BIRC5,CCL3,CDKN2A,DLGAP5,EPCAM,EZH2,JUN,KIAA0101,MCM2,MMP9,UBE2C

3,58E-03 14 APOE,AR,BCAM,BIRC5,CCL3,CDKN2A,DLGAP5,EPCAM,EZH2,JUN,KIAA0101,MCM2,MMP9,UBE2C

4,07E-03 21 APOE,AR,BCAM,BIRC5,CASP10,CCNB1,CDKN2A,COX1,DLGAP5,EPCAM,EZH2,F5,FASN,HSPE1,IDO1,JUN,KCNQ1OT1,MELK,MMP9,NDRG1,TK

4,21E-03 3 BIRC5,CDKN2A,MMP9

4,21E-03 3 BIRC5,CDKN2A,MMP9

4,24E-03 21 APOE,AR,BCAM,BIRC5,CASP10,CCNB1,CDKN2A,COX1,DLGAP5,EPCAM,EZH2,F5,FASN,HSPE1,IDO1,JUN,KCNQ1OT1,MELK,MMP9,NDRG1,TK1

4,87E-03 16 ADRB2,APOE,AR,BIRC5,CASP10,CCL3,CCL4,DEFA3,F5,FASN,HLA-DMA,HLA-DMB,ISG15,JUN,MMP9,SLC19A1

5,27E-03 4 BIRC5,CCNB1,CDKN2A,MMP9

ADAM8,APLN,APOE,AR,BCAM,BIRC5,CASP10,CCL3,CCNB1,CDKN2A,CENPU,COX1,DLGAP5,E

5,50E-03 33 PCAM,EZH2,F12,F5,FASN,HSPE1,IDO1,ISG15,JUN,KCNQ1OT1,MCM2,MELK,MMP11,MMP9,ND

RG1,PTK6,PTTG1,REG1A,STMN1,TK1

5,86E-03 14 APOE,AR,BCAM,BIRC5,CCL3,CDKN2A,DLGAP5,EPCAM,EZH2,JUN,KIAA0101,MCM2,MMP9,UBE2C

6,14E-03 9 AP1M2,AR,BIRC5,CCNB1,CDKN2A,EPCAM,EZH2,HPN,MMP9

6,14E-03 9 AP1M2,AR,BIRC5,CCNB1,CDKN2A,EPCAM,EZH2,HPN,MMP9

6,77E-03 13 ADRB2,APOE,AR,BIRC5,CASP10,CCL3,DEFA3,HLA-DMA,HLA-DMB,ISG15,JUN,MMP9,SLC19A1

6,80E-03 21 APOE,AR,BCAM,BIRC5,CASP10,CCNB1,CDKN2A,COX1,DLGAP5,EPCAM,EZH2,F5,FASN,HSPE1,IDO1,JUN,KCNQ1OT1,MELK,MMP9,NDRG1,TK1

7,02E-03 14 ADRB2,APOE,AR,BIRC5,CASP10,CCL3,CCL4,DEFA3,HLA-DMA,HLA-DMB,ISG15,JUN,MMP9,SLC19A1

7,14E-03 11 APLN,AR,BIRC5,CDKN2A,EPCAM,EZH2,MELK,MMP11,MMP9,NDRG1,TMSB15A

7,68E-03 2 AR,CDKN2A

$7,68 \mathrm{E}-03 \quad 2$ AR,CDKN2A

7,68E-03 2 CDKN2A,MCM2 
DOID:5113 nutritional deficiency disease

DOID:10534 stomach cance

DOID:3119 gastrointestinal system cancer

DOID:540 strabismus

DOID:9352 type 2 diabetes mellitus

DOID:1319 brain cancer

DOID:3652 Leigh disease

DOID:591 phobic disorder

DOID:8574 lichen disease

DOID:9201 lichen planus

DOID:74 hematopoietic system disease

DOID:3620 central nervous system cancer

DOID:848 arthritis

DOID:2876 laryngeal squamous cell carcinoma

DOID:178 vascular disease

DOID:10211 cholelithiasis

DOID:1279 ocular motility disease

DOID:319 spinal cord disease

DOID:11801 protein-energy malnutrition

DOID:2891 thyroid adenoma

DOID:3390 palmoplantar keratosis

DOID:4231 histiocytoma

DOID:5041 esophageal cancer

DOID:0060262 gallbladder disease

DOID:2596 larynx cancer

DOID:10923 sickle cell anemia

DOID:9834 hyperopia

DOID:4418 cutaneous fibrous histiocytoma

DOID:3376 bone osteosarcoma

DOID:263 kidney cancer

DOID:120 female reproductive organ cance

DOID:2871 endometrial carcinoma

DOID:305 carcinoma

DOID-0060089 endocrine organ benign neoplasm

DOID:3565 meningioma

DOID:4074 pancreas adenocarcinoma

DOID:5517 stomach carcinoma

DOID:1287 cardiovascular system disease

DOID:6713 cerebrovascular disease

DOID:2438 dermis tumor

DOID:3905 lung carcinom

DOID:0050134 cutaneous mycosis

DOID:10247 pleurisy

DOID:14283 primary hypertrophic osteoarthropathy

DOID:2231 factor XII deficiency

DOID:2451 protein S deficiency
7,78E-03 3 CDKN2A,CDKN3,MCM2

7,95E-03 13 AR,B3GAT1,BIRC5,CCL4,CDKN2A,EZH2,HPGD,ITPR3,JUN,MMP11,MMP9,PTTG1,REG1A

8,44E-03 26 APOE,AR,B3GAT1,BCAM,BIRC5,CCL3,CCL4,CCNB1,CDKN2A,DLGAP5,EPCAM,EZH2,HPGD,HS

26 PE1,ITPR3,JUN,KIAA0101,LGALS2,MCM2,MMP11,MMP9,NDRG1,PTTG1,REG1A,SOX4,UBE2C

8,88E-03 3 MMP11,MMP9,PHOX2A

9,51E-03 13 ADRB2,APLN,APOC1,APOE,AR,CA1,CDKN2A,F5,HLA-DMA,HLA-DMB,ITPR3,MMP9,PVT1

9,94E-03 4 APLN,BIRC5,MELK,MMP9

$1,01 \mathrm{E}-02-2 \mathrm{ND} 2, \mathrm{ND} 3$

$1,01 \mathrm{E}-02 \quad 2$ AR,CAMKK 2

$1,01 \mathrm{E}-02 \quad 2$ IDO1,MMP9

$1,01 \mathrm{E}-02 \quad 2$ IDO1,MMP9

1,02E-02 9 AR,BIRC5,CA1,F5,FABP1,FGG,MMP9,RECQL4,UBE2T

$1,04 \mathrm{E}-02-6$ APLN,BIRC5,CDKN2A,MELK,MMP11,MMP9

1,10E-02 13 ADRB2,APOE,AR,BIRC5,CASP10,CCL3,DEFA3,HLA-DMA,HLA-DMB,ISG15,JUN,MMP9,SLC19A1

$1,13 \mathrm{E}-02 \quad 3$ BIRC5,CCNB1,CDKN2A

1,22E-02 17 ADRB2,APLN,APOC1,APOE,CDKN2A,F5,FABP1,FGG,HSPE1,JUN,LGALS2,MMP9,ND1,ND2,PHOX2A,TK1,TRAP1

1,27E-02 3 APOC1,APOE,AR

1,27E-02 3 MMP11,MMP9,PHOX2A

1,27E-02 3 AR,MMP9,SLC19A1

$1,28 \mathrm{E}-02 \quad 2$ CDKN2A,MCM2

$1,28 \mathrm{E}-02 \quad 2$ CDKN2A,MCM

$1,28 \mathrm{E}-02 \quad 2$ CCL3,CCL3L3

$1,28 \mathrm{E}-02 \quad 2$ CDKN2A,MCM2

1,36E-02 9 AR,BIRC5,CCNB1,CDKN2A,EPCAM,MMP9,NDRG1,PTTG1,UBE2C

$1,42 \mathrm{E}-02 \quad 3$ APOC1,APOE,AR

1,45E-02 4 BIRC5,CCNB1,CDKN2A,MMP9

$1,57 \mathrm{E}-02 \quad 2$ ADRB2,BCAM,F5

1,57E-02 3 MMP11,MMP9,PHOX2A

$1,58 \mathrm{E}-02 \quad 2$ AR,MMP9

1,60E-02 5 CDKN2A,JUN,MMP9,RECQL4,SLC19A1

1,64E-02 9 AP1M2,AR,BIRC5,CCNB1,CDKN2A,EPCAM,EZH2,HPN,MMP9

1,72E-02 12 AR,BIRC5,CDKN2A,COX1,EPCAM,FASN,HSPE1,JUN,MCM2,MMP9,NDRG1,TK1

1,77E-02 7 AR,BIRC5,CDKN2A,COX1,HSPE1,MMP9,NDRG1

1,77E-02 16 ADAM8,BIRC5,CCL3,CDKN2A,EPCAM,F12,FASN,JUN,MMP11,MMP9,NDRG1,PTK6,PTTG1,REG1A,STMN1,TK1

1,79E-02 4 CDKN2A,MCM2,MMP9,PTTG1

1,79E-02 4 BIRC5,CDKN2A,MMP11,MMP9

1,79E-02 4 ADAM8,CDKN2A,JUN,NDRG1

1,81E-02 6 BIRC5,CCL4,CDKN2A,MMP9,PTTG1,REG1A

1,81E-02 18 ADRB2,APLN,APOC1,APOE,AR,CDKN2A,F5,FABP1,FGG,HSPE1,JUN,LGALS2,MMP9,ND1,ND2 PHOX2A,TK1,TRAP1

1,91E-02 3 APOE,F5,FABP1,LGALS2

$1,91 \mathrm{E}-02 \quad 2$ AR,MMP9

1,94E-02 8 ADRB2,BIRC5,CDKN2A,CTAG1A,MARCKSL1,MMP9,STMN1,TK1

$1,99 \mathrm{E}-02 \quad 1$ APOE

$1,99 \mathrm{E}-02 \quad 1$ MMP9

1,99E-02 1 HPGD

1,99E-02 1 F12

$1,99 \mathrm{E}-02$ 


\begin{tabular}{|c|c|c|c|}
\hline DOID:2519 & testicular disease & $1,99 \mathrm{E}-02$ & $1 \mathrm{AR}$ \\
\hline DOID:2732 & Rothmund-Thomson syndrome & $1,99 \mathrm{E}-02$ & 1 RECQL4 \\
\hline DOID:3540 & choroid plexus cancer & $1,99 \mathrm{E}-02$ & 1 BIRC5 \\
\hline DOID:3763 & hermaphroditism & $1,99 \mathrm{E}-02$ & $1 \mathrm{AR}$ \\
\hline DOID:4232 & extraosseous Ewing's sarcoma & $1,99 \mathrm{E}-02$ & $1 \mathrm{CDKN} 2 \mathrm{~A}$ \\
\hline DOID:4415 & fibrous histiocytoma & $1,99 \mathrm{E}-02$ & $1 \mathrm{MCM} 2$ \\
\hline DOID:4674 & androgen insensitivity syndrome & $1,99 \mathrm{E}-02$ & $1 \mathrm{AR}$ \\
\hline DOID:4985 & extraosseous Ewings sarcoma-primitive neuroepithelial tur & $1,99 \mathrm{E}-02$ & 1 CDKN2A \\
\hline DOID:5003 & eunuchism & $1,99 \mathrm{E}-02$ & $1 \mathrm{AR}$ \\
\hline DOID:5289 & uterus leiomyosarcoma & $1,99 \mathrm{E}-02$ & $1 \mathrm{CDKN} 2 \mathrm{~A}$ \\
\hline DOID:7475 & diverticulitis & $1,99 \mathrm{E}-02$ & 1 IDO1 \\
\hline DOID:8913 & dermatophytosis & $1,99 \mathrm{E}-02$ & $1 \mathrm{APOE}$ \\
\hline DOID:9362 & status asthmaticus & $1,99 \mathrm{E}-02$ & 1 ADRB2 \\
\hline DOID:5223 & infertility & 2,03E-02 & 4 AR,BIRC5,CRISP2,F5 \\
\hline DOID:184 & bone cancer & $2,05 \mathrm{E}-02$ & 6 AR,CDKN2A,JUN,MMP9,RECQL4,SLC19A1 \\
\hline DOID:170 & endocrine gland cancer & $2,09 \mathrm{E}-02$ & 13 ABCC5,ADAM8,APOC1,B3GAT1,BIRC5,CCNB1,CDKN2A,JUN,MCM2,MMP11,MMP9,NDRG1,PTTG1 \\
\hline DOID:1891 & optic nerve disease & $2,26 \mathrm{E}-02$ & 2 ND1,ND6 \\
\hline DOID:9279 & hyperhomocysteinemia & 2,26E-02 & 2 APOE,F5 \\
\hline DOID:9840 & esotropia & $2,26 \mathrm{E}-02$ & 2 MMP11,MMP9 \\
\hline DOID:3347 & osteosarcoma & 2,27E-02 & 5 CDKN2A,JUN,MMP9,RECQL4,SLC19A1 \\
\hline DOID:2394 & ovarian cancer & 2,31E-02 & 6 BIRC5,CDKN2A,EPCAM,MCM2,MMP9,TK1 \\
\hline DOID:1380 & endometrial cancer & 2,34E-02 & 7 AR,BIRC5,CDKN2A,COX1,HSPE1,MMP9,NDRG1 \\
\hline DOID:363 & uterine cancer & 2,34E-02 & 7 AR,BIRC5,CDKN2A,COX1,HSPE1,MMP9,NDRG1 \\
\hline DOID:0060085 & organ system benign neoplasm & 2,37E-02 & 9 AR,BCAM,BIRC5,CDKN2A,ESM1,MCM2,MMP9,NDRG1,PTTG1 \\
\hline DOID:65 & connective tissue disease & 2,49E-02 & 17 ADRB2,APOE,AR,BIRC5,CASP10,CCL3,CCL4,DEFA3,F5,FASN,HLA-DMA,HLA-DMB,ISG15,ITPR3,JUN,MMP9,SLC19A1 \\
\hline DOID:201 & connective tissue cancer & 2,63E-02 & 8 AR,BIRC5,CDKN2A,JUN,MCM2,MMP9,RECQL4,SLC19A1 \\
\hline DOID:4905 & pancreatic carcinoma & 2,73E-02 & 5 ABCC5,ADAM8,CDKN2A,JUN,NDRG1 \\
\hline DOID:4645 & retinal cancer & 2,91E-02 & 3 AR,CDKN2A,EPCAM \\
\hline DOID:768 & retinoblastoma & 2,91E-02 & 3 AR,CDKN2A,EPCAM \\
\hline DOID:771 & retinal cell cancer & 2,91E-02 & 3 AR,CDKN2A,EPCAM \\
\hline DOID:9835 & refractive error & 2,91E-02 & 3 MMP11,MMP9,PHOX2A \\
\hline DOID:0080014 & chromosomal disease & $2,98 \mathrm{E}-02$ & 5 APOE,AR,FAAH,SLC19A1,STMN1 \\
\hline DOID:700 & mitochondrial metabolism disease & 3,04E-02 & $2 \mathrm{ND} 2, \mathrm{ND} 3$ \\
\hline DOID:9588 & encephalitis & $3,04 \mathrm{E}-02$ & 2 APOE,CCL3 \\
\hline DOID:326 & ischemia & 3,05E-02 & 4 APOE,HSPE1,MMP9,TRAP1 \\
\hline DOID:0060116 & sensory system cancer & $3,21 \mathrm{E}-02$ & 4 AR,CDKN2A,EPCAM,EZH2 \\
\hline DOID:2174 & ocular cancer & 3,21E-02 & 4 AR,CDKN2A,EPCAM,EZH2 \\
\hline DOID:870 & neuropathy & $3,22 \mathrm{E}-02$ & 6 MMP11,MMP9,ND1,ND6,NDRG1,PHOX2A \\
\hline DOID:4866 & salivary gland adenoid cystic carcinoma & $3,46 \mathrm{E}-02$ & 2 CDKN2A,SOX4 \\
\hline DOID:0050624 & gastrointestinal system benign neoplasm & $3,62 \mathrm{E}-02$ & 3 BIRC5,CDKN2A,NDRG1 \\
\hline DOID:0060086 & female reproductive organ benign neoplasm & $3,62 \mathrm{E}-02$ & 3 AR,CDKN2A,PTTG1 \\
\hline DOID:0060095 & uterine benign neoplasm & 3,62E-02 & 3 AR,CDKN2A,PTTG1 \\
\hline DOID:13223 & uterine fibroid & $3,62 \mathrm{E}-02$ & 3 AR,CDKN2A,PTTG1 \\
\hline DOID:552 & pneumonia & $3,74 \mathrm{E}-02$ & 4 APOE,CDKN2A,FGG,MMP9 \\
\hline DOID:8692 & myeloid leukemia & $3,84 \mathrm{E}-02$ & 9 ABCA3,BIRC5,CCL3,CCNB1,CDKN2A,IDO1,JUN,MMP9,PTTG1 \\
\hline DOID:3829 & pituitary adenoma & $3,88 \mathrm{E}-02$ & 3 CDKN2A,MMP9,PTTG1 \\
\hline DOID:11612 & polycystic ovary syndrome & 3,93E-02 & 4 ADRB2,AR,F5,MMP9 \\
\hline DOID:0060443 & endothelial dystrophy & 3,93E-02 & 1 BIRC5 \\
\hline
\end{tabular}




\begin{tabular}{|c|c|c|c|}
\hline DOID:10541 & microinvasive gastric cancer & 3,93E-02 & 1 REG1A \\
\hline DOID:10863 & paralytic squint & 3,93E-02 & 1 PHOX2A \\
\hline DOID:10869 & fourth cranial nerve palsy & 3,93E-02 & 1 PHOX2A \\
\hline DOID:11555 & Fuchs' endothelial dystrophy & $3,93 \mathrm{E}-02$ & 1 BIRC5 \\
\hline DOID:13620 & patent foramen ovale & 3,93E-02 & $1 \mathrm{~F} 5$ \\
\hline DOID:14748 & Sotos syndrome & $3,93 \mathrm{E}-02$ & $1 \mathrm{~F} 12$ \\
\hline DOID:1659 & supratentorial cancer & 3,93E-02 & 1 BIRC5 \\
\hline DOID:2216 & factor $\mathrm{V}$ deficiency & $3,93 \mathrm{E}-02$ & $1 \mathrm{~F} 5$ \\
\hline DOID:2671 & transitional cell carcinoma & 3,93E-02 & 1 BIRC5 \\
\hline DOID:318 & progressive muscular atrophy & 3,93E-02 & $1 \mathrm{AR}$ \\
\hline DOID:3362 & coronary aneurysm & $3,93 \mathrm{E}-02$ & 1 MMP9 \\
\hline DOID:3500 & gallbladder adenocarcinoma & 3,93E-02 & $1 \mathrm{CDKN} 2 \mathrm{~A}$ \\
\hline DOID:3541 & cerebral ventricle cancer & $3,93 \mathrm{E}-02$ & 1 BIRC5 \\
\hline DOID:368 & cerebrum cancer & 3,93E-02 & 1 BIRC5 \\
\hline DOID:3717 & gastric adenocarcinoma & $3,93 \mathrm{E}-02$ & 1 REG1A \\
\hline DOID:4012 & papillary transitional carcinoma & 3,93E-02 & 1 BIRC5 \\
\hline DOID:4449 & macular retinal edema & $3,93 \mathrm{E}-02$ & $1 \mathrm{APOE}$ \\
\hline DOID:5165 & uterine corpus sarcoma & 3,93E-02 & $1 \mathrm{CDKN} 2 \mathrm{~A}$ \\
\hline DOID:5199 & ureteral obstruction & $3,93 \mathrm{E}-02$ & 1 FABP1 \\
\hline DOID:5200 & urinary tract obstruction & 3,93E-02 & 1 FABP1 \\
\hline DOID:6929 & retinal edema & $3,93 \mathrm{E}-02$ & $1 \mathrm{APOE}$ \\
\hline DOID:801 & hemarthrosis & 3,93E-02 & 1 MMP9 \\
\hline DOID:899 & choledochal cyst & 3,93E-02 & 1 REG1A \\
\hline DOID:9460 & uterine corpus cancer & 3,93E-02 & $1 \mathrm{CDKN} 2 \mathrm{~A}$ \\
\hline DOID:9499 & disseminated eosinophilic collagen disease & 3,93E-02 & 1 BIRC5 \\
\hline DOID:9651 & systolic heart failure & 3,93E-02 & 1 MMP9 \\
\hline DOID:9768 & heart aneurysm & 3,93E-02 & 1 MMP9 \\
\hline DOID:10763 & hypertension & $4,27 \mathrm{E}-02$ & 10 ADRB2,APLN,APOE,CDKN2A,F5,JUN,MMP9,ND1,ND2,PHOX2A \\
\hline DOID:10124 & corneal disease & $4,37 \mathrm{E}-02$ & 2 BIRC5,MMP9 \\
\hline DOID:381 & arthropathy & $4,37 \mathrm{E}-02$ & 2 CCL4,MMP9 \\
\hline DOID:0050622 & reproductive organ benign neoplasm & $4,41 \mathrm{E}-02$ & 3 AR,CDKN2A,PTTG1 \\
\hline DOID:127 & leiomyoma & $4,41 \mathrm{E}-02$ & 3 AR,CDKN2A,PTTG1 \\
\hline DOID:0070004 & myeloma & $4,57 \mathrm{E}-02$ & 7 AR,BIRC5,CCL3,CCL4,CDKN2A,F5,PTTG1 \\
\hline DOID:4960 & bone marrow cancer & $4,57 \mathrm{E}-02$ & 7 AR,BIRC5,CCL3,CCL4,CDKN2A,F5,PTTG1 \\
\hline DOID:2377 & multiple sclerosis & $4,77 \mathrm{E}-02$ & 6 APOE,IDO1,MMP9,ND1,ND5,STMN1 \\
\hline DOID:0050615 & respiratory system cancer & $4,81 \mathrm{E}-02$ & 13 ADAM8,ADRB2,BIRC5,CCNB1,CDKN2A,CTAG1A,HPGD,MARCKSL1,MMP9,SLC19A1,STMN1,TK1,UBE2T \\
\hline DOID:37 & skin disease & 4,83E-02 & 8 ADRB2,APOC1,CCL3,CCL3L3,F12,IDO1,MMP9,RECQL4 \\
\hline DOID:3996 & urinary system cancer & 4,83E-02 & 10 AP1M2,AR,BIRC5,CCNB1,CDKN2A,EPCAM,EZH2,HPN,MMP9,ZDHHC11 \\
\hline DOID:225 & syndrome & $4,85 \mathrm{E}-02$ & 11 ADRB2,AR,CASP10,CRISP2,F12,F5,HPGD,KCNQ1OT1,MLPH,MMP9,RECQL4 \\
\hline DOID:161 & keratosis & $4,86 \mathrm{E}-02$ & 2 CCL3,CCL3L3 \\
\hline DOID:14330 & Parkinson's disease & 4,93E-02 & 5 APOE,CDKN2A,ND1,ND2,ND5 \\
\hline
\end{tabular}

